Portland State University

PDXScholar

Spring 5-26-2016

\title{
Intercultural Interactions Among Burmese Refugees in Multicultural Middle School Classrooms
}

Matthew Carl McParker

Portland State University

Follow this and additional works at: https://pdxscholar.library.pdx.edu/open_access_etds

Part of the Bilingual, Multilingual, and Multicultural Education Commons Let us know how access to this document benefits you.

\section{Recommended Citation}

McParker, Matthew Carl, "Intercultural Interactions Among Burmese Refugees in Multicultural Middle School Classrooms" (2016). Dissertations and Theses. Paper 2942.

https://doi.org/10.15760/etd.2937

This Dissertation is brought to you for free and open access. It has been accepted for inclusion in Dissertations and Theses by an authorized administrator of PDXScholar. Please contact us if we can make this document more accessible: pdxscholar@pdx.edu. 
Intercultural Interactions Among Burmese Refugees in Multicultural Middle School Classrooms

\author{
by \\ Matthew Carl McParker
}

A dissertation submitted in partial fulfillment of the requirements for the degree of

\author{
Doctor of Education \\ in \\ Educational Leadership: Curriculum and Instruction
}

Dissertation Committee:

Micki M. Caskey, Chair

Swapna Mukhopadhyay

Dannelle Stevens

Sharon Carstens

Portland State University

2016 
(C) 2016 Matthew Carl McParker 


\begin{abstract}
The largest refugee group entering the United States in recent years is from Burma. Refugee students face a daunting set of challenges, from language and cultural differences to living in poverty, in becoming successful in their new homes. To be successful in schools and gain cultural and social capital, refugee students must learn and internalize the specific norms of their classrooms. In middle school, students are particularly reliant upon their peers for support, making peer interactions especially important. In multicultural settings, students have ample opportunities for intercultural interactions, which can help refugee students navigate their new settings and become more successful. Unfortunately, there has been little research on the experiences of Burmese refugee students in classrooms in the United States.

I used a qualitative, transcendental phenomenological approach to study how three female Burmese refugee students experienced multicultural middle school classrooms in the United States, especially their intercultural interactions, through interviews, observations, and stimulated recall.

The participants reported wanting to understand what they were learning, stay on task, and be kind to other students. Those traits developed from their experiences in their countries of origin and combined to create a picture of what a good student should be. In observations, students acted out their ideas of what it meant to be a good student. Their intercultural interactions in class reaffirmed their identities as good students.
\end{abstract}


Implications based on the findings include setting up intentional intercultural interactions with a diverse group of students in classrooms with multicultural approaches and that researchers examine the experiences of various groups of marginalized students while accounting for the context in which they learn and acknowledging a multifaceted view of adolescent identity development. 
Table of Contents

Abstract

List of Tables

Chapter 1: Statement of the Research Problem

Background of the Problem 2

Statement of the Research Problem 9

Significance of the Research Problem 11

Presentation of Methods and Research Questions $\quad 12$

Definition of Key Concepts 13

Chapter 2: Review of the Literature $\quad 19$

$\begin{array}{ll}\text { Theoretical Framework } & 19\end{array}$

Theory of Field 21

Theory of Social and Cultural Reproduction 27

Critique of Bourdieu's Theories $\quad 28$

Summary of Theoretical Framework $\quad 30$

Review of the Research Literature $\quad 30$

Middle school and identity formation 31

Ethnic Identity Formation $\quad 36$

Multicultural Context 43

Intercultural Interaction $\quad 51$

Intergroup Contact 53

Intercultural Communication $\quad 60$

$\begin{array}{ll}\text { Refugee Students } & 65\end{array}$

Community-based and School-wide Programs 70

Classroom-based Practices 74

Female Refugee Students $\quad 78$

Burmese Refugees $\quad 80$

Summary of Literature Review $\quad 86$

Review of the Methodological Literature $\quad 90$

Interview $\quad 93$

$\begin{array}{lr}\text { Observation } & 98\end{array}$

Stimulated Recall 101

Summary of Methodological Review 109

Chapter 3: Methods $\quad 112$

$\begin{array}{ll}\text { Research Methods } & 115\end{array}$

$\begin{array}{lr}\text { Participants } & 120\end{array}$

Sampling $\quad 120$

$\begin{array}{lr}\text { Procedures } & 126\end{array}$

$\begin{array}{ll}\text { Interviews } & 127\end{array}$

Observations 130

Stimulated Recall 131

$\begin{array}{lr}\text { Instruments and Measures } & 135\end{array}$ 
Establishing Validity $\quad 137$

Role of the Researcher $\quad 141$

Data Collection and Analysis 143

$\begin{array}{lr}\text { Conclusion } & 146\end{array}$

Chapter 4: Results and Analysis $\quad 149$

Analysis of Data $\quad 153$

Presentation of Results $\quad 154$

Focus on Understanding 157

Summary 165

$\begin{array}{ll}\text { Staying on Task } & 166\end{array}$

Summary $\quad 181$

Being Kind to Others $\quad 182$

Summary 193

Connect to Research Questions 194

Experiences of Burmese Students in Multicultural Middle School

$\begin{array}{ll}\text { Classrooms } & 194\end{array}$

Experiences of Burmese Students in Intercultural Interactions 198

Effects of Intercultural Interactions on Burmese Students'

Experiences in Multicultural Middle School Classrooms 202

$\begin{array}{ll}\text { Interpretation of Findings } & 206\end{array}$

Bracketing Out the Researcher's Experience 206

Experiences of the Participants 210

$\begin{array}{ll}\text { Limitations of Study } & 212\end{array}$

Few Participants $\quad 213$

Limited Time $\quad 213$

Limited Classes $\quad 214$

Influence of the Teacher $\quad 215$

Potential for Fabrication $\quad 215$

Limitations of Methods $\quad 216$

Chapter 5: Discussion and Conclusion $\quad 217$

$\begin{array}{ll}\text { Synthesis of Findings } & 220\end{array}$

Situated in Larger Context 222

$\begin{array}{ll}\text { Implications } & 230\end{array}$

Implications for Practice $\quad 231$

Implications for Research $\quad 234$

Summary and Conclusion 236

$\begin{array}{ll}\text { References } & 238\end{array}$

$\begin{array}{ll}\text { Appendices } & 260\end{array}$

Appendix A Semi-Structured Interview Protocol 260

Appendix B Observation Protocol $\quad 262$

Appendix C Descriptive Codes 263 


\section{List of Tables}

Table 1. Ethnic Identity Formation - Marcia (1980)

Table 2. Primary and Secondary Methods for Addressing Each Research Question

Table 3. Initial Themes Aligned to Categories 


\section{Chapter 1: Statement of the Research Problem}

Early in the 2012-2013 school year, before I knew all my students well, I called on one particular student to answer a question. Tae Moo (pseudonym) had moved from a refugee camp in Thailand a few years prior. Instead of answering the question, she turned to a friend from the same cultural background, Paw Htoo (pseudonym), who answered the question for her. Paw Htoo moved away a few weeks later. When I next called on Tae Moo, I was curious what her reaction would be, without her friend as support. Despite being seated next to very capable students, none of whom was from the same cultural background as she, Tae Moo did not ask any of them for help. Instead of answering or asking for help, she sat silently smiling until I coached her to respond. That incident made me wonder what made it easy for Tae Moo to ask a peer from the same cultural background, but difficult to ask others. Language was likely a factor, but Tae Moo spoke competent English. Could it have been their shared experience of being Burmese refugee students? Did the student feel disconnected from other students in the classroom? What was it that allowed her to speak openly to another student with a similar background, but not to other capable students?

In my classes, students often come from 15 or more cultures and have ample opportunities to interact with others from other cultural backgrounds. Evidence shows interaction with peers is beneficial for students (Chan, 2007; Ryan \& Patrick, 2001; Stoughton \& Sivertson, 2005; Wenzel \& Watkins, 2002). When those interactions are with peers from other cultural backgrounds, there are further benefits in overcoming challenges, such as mutual understanding, reduced prejudice, and secure identity 
formation, especially for marginalized students such as refugees (Gurin, Nagda, \& Sorensen, 2011; Ryan \& Patrick, 2001; Wentzel, Baker, \& Russell, 2012; Yoon, 2012). Despite the benefits of interacting with peers from other cultures, students have tended not to take advantage of their opportunities to engage in intercultural interactions (Halualani, Chitgopekar, Huynh, Morrison, \& Dodge, 2004; Volet \& Ang, 1998). I saw Burmese refugee students at my school interacting consistently with a small group of peers who were mostly Burmese refugees themselves. Though they were typically goodnatured, kind, hard-working students, they tended to struggle academically. I often wondered how much better they would do if they were to increase their interactions with peers from other cultural backgrounds. The purpose of this study was to explore the experiences, specifically the intercultural interactions, of Burmese refugee students in multicultural middle school classrooms.

\section{Background of the Problem}

In this section, I detail the context of my social studies classroom and explain some of the challenges Burmese refugee students face in multicultural middle school classes. Then I highlight the importance of peer interaction in middle school. Finally, I explore how intercultural interactions may help Burmese refugee students overcome some of their challenges.

During the study, I taught at an urban middle school in the Northwestern United States with about 900 students in grades 6 through 8 . Approximately $90 \%$ of the student population was on free or reduced-price lunch, with about 35 different languages spoken. Prior to the study, in the 2011-2012 school year, the English Language Learners (ELL) 
department had five teachers, allowing for a variety of sheltered classes. Because many students in the ELL program were in sheltered classes, they were not enrolled in mainstream classes. When I conducted the study in the 2014-2015 school year, with approximately the same number of students in our ELL program, the department had only two teachers. As a result, the number of recent immigrant and refugee students in mainstream classes has increased. In 2011-2012, far less than $20 \%$ of my students were in ELL classes. Since then, $20 \%$ or more of my students have been in ELL classes. This trend has been relatively consistent across content areas in the school.

As a part of my regular teaching, I expected all of my students to interact with all of my other students. Most of the teachers in my building have had the same expectation. Especially in middle school, these interactions help students learn and form their identities (Chan, 2007; Stoughton \& Sivertson, 2005). According to McLean, Breen, and Fournier (2010), "Adolescence is a time when identity development takes center stage" (p. 184). Habermas and Bluck (2000) and McAdams (2001) concurred that the identity formation process begins in adolescence. To form these identities, relationships and interactions with others are crucial (McLean, Breen, \& Fournier, 2010).

In addition to helping individuals form strong identities, interactions among different groups of students can help these groups understand each other and work toward common goals (Gurin et al., 2011), allowing for multicultural understanding among all groups. Immigrant and refugee students stand to benefit the most from interactions with others, as they must navigate typical adolescent struggles while learning the norms of an 
unfamiliar society (deAnda, 1984; Lee \& Koro-Ljungberg, 2007; McBrien, 2005; Pinson \& Arnot, 2007; Valenta, 2010).

According to the United Nations High Commissioner for Refugees (UNHCR), and further clarified by the 1969 Organization of African Unity (OAU) and Article III of the 1984 Cartagena Declaration on Refugees, refugees are groups of people who have been forced out of their countries of origin. Valenta (2010) distinguished refugees from immigrants in how they identify as being marginalized in the larger society. Refugees' past history, having been forced out of their countries of origin, significantly contributes to their sense of marginalization, and, thus, their overall identities. Educators need to address the struggles of refugees in school because "Of the over 15 million refugee and asylum-seekers in the world, $41 \%$ are children under 18 years of age" (McCarthy \& Vickers, 2012, p. xxiii). Since 2012, the number of child refugees has risen. According to the UNHCR (2015), in $201451 \%$ of all refugees were children.

Once they arrive in the United States, refugees face many challenges, including navigating differences from the dominant culture, managing past trauma, learning English, lack of education, and living in poverty (Dryden-Peterson, 2015; McBrien, 2005; Valenta, 2010). Refugee students must learn how to be academically and socially successful in interacting with others while learning how to navigate an unfamiliar society (McBrien, 2005). The combination of academic and social pressures with an unfamiliar society often leads to marginalization (an exclusionary process by which some groups of people are denied access to the benefits of the hegemonic culture), discrimination, and struggles in academic achievement (McBrien, 2005; Pugh, Every, \& Hattam, 2012). 
Differences between refugees' cultures and the hegemonic culture, coupled with marginalization, make it difficult for refugees to access the hegemonic culture (McBrien, 2005; Valenta, 2010).

Complicating matters, refugees often come from traumatic situations that many other students have not experienced (McBrien, 2005). Furthering their marginalization from the hegemonic culture, language differences can negatively affect students' education and social integration, which in turn can affect their senses of identity (Pugh et al., 2012). In addition, refugee students often live in poverty (McBrien, 2005), which is correlated with low achievement in school (Pugh et al., 2012). Dávila (2012) found that despite efforts from both the school and students, institutional barriers keep refugee students from being successful in school. Even if the school does a great deal of work to positive and welcoming environment, there is no guarantee that the refugee students will benefit. Despite efforts by a community to create a welcoming environment, an exclusionary effect may remain for immigrant students (Gitlin, Buendía, Crosland, \& Doumbia, 2003). Portes and Zhou (1993) claimed that refugee children arriving since the 1970s are less likely than those who came before to access the middle-class, hegemonic culture. Students may react by creating identities in opposition to the hegemonic culture (Ogbu, 2004).

One of the largest refugee groups entering the United States in recent years is from Burma. Between 2009 and 2011, nearly 52,000 Burmese refugees entered the United States. In 2011, they comprised over 30\% of all refugees admitted (Martin \& Yankay, 2012). Globally, Burmese refugees are the seventh largest group receiving 
refugee status (UNHCR, 2015). Specifically in Oregon, the Burmese were one of the five largest refugee groups to enter in 2009 even though they were first recognized as a distinct refugee group by the state in 2008 (Oregon Department of Human Services, n.d.). Though the number of refugees from Burma has leveled off since 2012 (UNHCR, 2015), many children who arrived through 2011 were still in school during the 2014-2015 school year. Many of those who arrived as elementary students would have been in middle school at the time I conducted my study.

The Burmese are people from Burma, also called Myanmar, a Southeast Asian country bordering Bangladesh, India, China, Laos, and Thailand. The country had been under military rule since 1962, and became "one of the world's least developed and least free countries" (Ranard \& Barron, 2007, p. 1). Since 2011, there has been a move toward democratic rule, culminating in elections in 2015 (Nilsen, 2013). Many different ethnic groups live in Burma, several of whom have resettled as refugees in the United States. The largest ethnic refugee group is the Burmans (Ranard \& Barron, 2007), but others include Karen, Karenni, Rohingya, Shan, and Chin. For the purposes of this study, due to their similar experiences of being refugees resettled to the United States, I am grouping together all of the Burmese ethnic groups rather than discussing them separately. The different groups all came from similar, sometimes the same, refugee camps in countries that neighbor Burma. They were often placed together in the United States, and the students in my school typically associated with each other regardless of their specific ethnic groups. 
BURMESE REFUGEE STUDENT INTERACTION

While all of the challenges refugee students face can seem bleak, it is possible for students to access the benefits of the hegemonic culture. Peer interaction can be a way for marginalized students to overcome their challenges, especially if those interactions are with peers from other cultural backgrounds (Gurin et al., 2011; Ryan \& Patrick, 2001; Wentzel et al., 2012; Yoon, 2012). Reliance on peers for support is especially important in middle school (Kiefer, Matthews, Montesino, Arango, \& Preece, 2013). It is through interactions with peers that middle school students learn and form their identities (Stoughton \& Sivertson, 2005; Wentzel \& Watkins, 2002). According to Wentzel, Baker, and Russell (2012), peer support in adolescence plays a larger role in encouraging academic success than either teacher or parent support. Even though Wang and Eccles (2012) said teacher support may have a larger impact than peer support on emotional and cognitive engagement, they stated that positive peer interactions equate with positive school engagement. Ryan and Patrick (2001) also found that peer interaction, in a supportive environment, is a major factor in positive patterns of learning. Phinney, Jacoby, and Silva (2007) argued that students with secure ethnic identities have positive attitudes toward other cultural groups, which may be influenced by opportunities for intercultural interaction. If positive peer interactions leads to secure identity formation, and secure ethnic identity leads to positive attitudes toward students from other cultures, it follows that positive interactions with students from other cultures might lead to more secure identity formation and better learning.

I have observed that refugee students in my school, particularly those from Burma, do not often interact with students from other cultural backgrounds. This 
phenomenon does not appear to be isolated to my school setting. According to Halualani, Chitgopekar, Huynh, Morrison, and Dodge (2004) and Volet and Ang (1998), students in multicultural settings tend to have very limited intercultural interactions. Typically, students interact with students from no more than one cultural group aside from their own (Halualani et al., 2004; Volet \& Ang, 1998). One reason for the lack of intercultural interactions may be that students have different understandings of what exactly constitutes intercultural interactions and how to make sense of them when they happen (Halualani, 2010). Though each of the above studies was conducted with university students as the target population, middle school students also limit their interactions with students from other cultural backgrounds. Lee and Chen (2000) found that the longer seventh and eighth grade students have been in their host country, the more competent they feel in communication. According to Lee and Chen (2000), competence in the host country communication, which is the ability "to decode and encode information in accordance with the communication practices of the host culture" (p. 766), comes with increased intercultural communication. When interactions between students do occur, though, students from the host culture often dominate the interactions (Lee \& Chen, 2000). Though not directly stating that middle school immigrant students limit their interactions with students from other cultural backgrounds, the results from their study indicate this to be the case, especially among recent immigrants.

If Burmese refugee students' classrooms could be structured to encourage productive interactions with students from other cultures, perhaps they would be able to gain confidence in their communication competence and learn how to navigate an 
unfamiliar society. By gaining confidence in the communication and in navigating their new society, refugees can form secure identities (Phinney, Jacoby, \& Silva, 2007), be more engaged in school (Wang \& Eccles, 2012), and improve their academic outcomes (Ryan \& Patrick, 2001; Wenzel et al., 2012).

In the next section, I explain the major benefits of students engaging in intercultural interaction and why it is important to study how one marginalized group, Burmese refugee students in middle school, experiences those interactions.

\section{Statement of the Research Problem}

Refugee students enter school in the United States facing a variety of challenges, from unfamiliarity with the hegemonic culture (McBrien, 2005; Pugh et al., 2012; Valenta, 2010), dealing with past trauma (McBrien, 2005), unfamiliarity with the host language (Pugh et al., 2012), living in poverty (McBrien, 2005; Pugh et al., 2012), educational inequities (Dryden-Peterson, 2015), and various institutional barriers that are exclusionary (Dávila, 2012; Gitlin et al., 2003). As a result, many students rebel (Ogbu, 2004) and have a difficult time accessing the benefits of the hegemonic culture (Portes \& Zhou, 1993). Even though interacting with peers, especially those from other cultural backgrounds, helps students form secure identities (Phinney et al., 2007), increases their engagement in school (Wang \& Eccles, 2012), and improves their academic outcomes (Ryan \& Patrick, 2001; Wenzel et al., 2012), Burmese refugee students in the middle school where I teach limit their interactions with students from other cultural backgrounds. Researchers have observed the same phenomenon elsewhere, concluding that students tend to interact with students of their own cultural background and perhaps 
one other (Halualani et al., 2004; Volet \& Ang, 1998). One reason students may limit their interactions is that people have differing versions of what constitutes intercultural interaction and how to make sense of the interactions when they happen (Halualani, 2010), which might lead to confusion. In my multicultural middle school, the problem is Burmese refugee students were not interacting with students from other cultural backgrounds enough to access the benefits that come with intercultural interaction. This problem led me to study the experiences female Burmese refugee students had in their multicultural middle school, specifically their experiences in intercultural interaction.

Though ample research exists regarding the challenges refugees face in classrooms (McBrien, 2005), little research specifically addresses how refugees overcome those challenges. Most of the literature, regarding both challenges and solutions, originates from outside the United States. In addition, very few studies have examined the experiences of Burmese refugee students or female refugee students. Despite the lack of research on how refugee students can be successful, many studies made clear the benefits of students interacting with other students. According to Ryan and Patrick (2001), peer interaction in a supportive environment is a major factor in positive patterns of learning. Phinney et al. (2007) argued intercultural interaction helps students form secure identities, leading to more positive views of diverse cultural groups. Gurin, Nagda, and Sorensen (2011) found that intercultural interaction leads to greater mutual understanding, greater understanding of inequality, and increased motivation to combat inequality. 
According to Halualani et al. (2004) and Volet and Ang (1998), students in multicultural settings do not interact with a wide range of peers. Though their studies both examine students in college, I observed the same phenomenon in the middle school where I teach. Even though engaging in intercultural interaction can be beneficial, especially to marginalized populations such as Burmese refugees, students tended to limit their interactions with peers to those of only one or two different cultures. When they limit their interactions, refugee students miss the benefits that Ryan and Patrick (2001) and Gurin et al. (2011) pointed out. In addition, when people engage in intercultural interaction, they have different definitions and interpretations of those interactions (Halualani, 2010). Because peer interaction is especially important in middle school (Chan, 2007; Kiefer et al., 2013; Stoughton \& Sivertson, 2005), investigating the experiences of one particular group of marginalized students may help teachers and researchers understand how they can best encourage more intercultural interaction and ultimately help all students.

\section{Significance of the Research Problem}

Because of their recent entry and large numbers, Burmese refugees provide a relevant lens through which to examine the experience of refugees and other marginalized populations. Middle school is an important time for students in general, and refugees in particular, because of students' reliance on their peers for support (Chan, 2007; Kiefer et al., 2013; Stoughton \& Sivertson, 2005). In multicultural classrooms, interactions with students from other cultural backgrounds can lead to greater understanding between cultures (Gurin et al., 2011), help refugee students adjust to an 
unfamiliar environment (Pugh et al., 2012; Taylor \& Sidhu, 2012), learn in various content areas (Alfassi, 2009; Gersten \& Baker, 2000; Janzen, 2008; Piccolo, Harbaugh, Carter, Capraro, \& Capraro, 2008), and form identities (Stoughton \& Sivertson, 2005) while decreasing marginalization from the hegemonic culture (den Brok, Fisher, Rickards, \& Bull, 2006; McBrien, 2005). Refugee students who do not participate in interactions with students from other cultures may have a particularly difficult time overcoming their challenges, such as unfamiliarity with the hegemonic culture and low academic achievement (McBrien, 2005; Valenta, 2010).

Marginalized students may be able to overcome their challenges through intercultural interaction. This study may help teachers and researchers begin to understand the intercultural interactions of one marginalized population, which may eventually help teachers structure classes to provide opportunities for more frequent intercultural interactions and that those interactions are maximally effective.

\section{Presentation of Methods and Research Questions}

In this study, I pursued three research questions.

1. What were the experiences of Burmese refugees in multicultural middle school classrooms?

2. How did Burmese refugee students experience interactions with students from other cultural backgrounds?

3. How did the interactions Burmese refugee students have with students from other cultural backgrounds affect their school experiences, including learning and identity formation? 
Because I explored the experiences of a particular group of students, I used qualitative methods. I interviewed students, videotaped observations in the classroom, and conducted stimulated recall sessions of the observations. The interviews were transcribed, coded, and member-checked for accuracy. I compared the interview data to the observations to get a fuller picture of Burmese students' experiences. To complete the picture, I conducted stimulated recall sessions, in which I asked students to discuss their experiences in various interactions with students from other cultural backgrounds.

I established validity through several methods. First, I investigated the issue using three separate, but complementary, approaches (i.e., observation, interviews, stimulated recall) with direct transcriptions as well as field notes. I videotaped the observation sessions and students discussed their experiences in those observations during stimulated recall sessions. I used member checking of the transcribed interviews to ensure my data matched the intentions of the participants. I had intensive investment in the project to check my data and to search for discrepant data. I also reported the data thoroughly, using verbatim quotes as much as possible. I included a discussion of my background so as to explain any potential biases.

\section{Definitions of Key Concepts}

Interaction. I use interaction to refer to a type of communication between people typified by reciprocal exchange of words and/or gestures. According to HosseiniMaasoum (2012), student-centered interactions are crucial for student learning. For knowledge construction to occur, students must actively participate. Especially in middle school, these interactions help students learn and form their own identities (Chan, 2007; 
Stoughton \& Sivertson, 2005). Interactions among different groups of students can help these groups understand each other and work toward common goals (Gurin et al., 2011), allowing for multicultural understanding among all groups. Immigrant and refugee students stand to benefit the most from interactions with others, as they must navigate typical adolescent struggles while learning the norms of an unfamiliar society (deAnda, 1984; Lee \& Koro-Ljungberg, 2007; McBrien, 2005; Pinson \& Arnot, 2007; Valenta, 2010).

Refugee. Refugees are groups of people from non-dominant cultures who fit the definition of a refugee as set out by the United Nations High Commissioner for Refugees (UNHCR). Currently, the UNHCR bases their definition on Article I of the Convention Relating to the Status of Refugees, which states that a refugee is anyone who cannot return to his or her country, "owing to well-founded fear of being persecuted for reasons of race, religion, nationality, membership of a particular social group or political opinion..." (UNHCR, 2005, p. 5). Furthermore, the 1969 Organization of African Unity (OAU) Convention added that refugee status applies to those who, due to external forces (e.g., foreign invasion, occupation) are forced to leave their country of origin. Article III of the 1984 Cartagena Declaration on Refugees broadened the definition of refugee to those who fear for their lives for human rights violations, general violence within the country, or threat of foreign invasion (UNHCR, 2005).

Burmese. The Burmese are people from Burma, also called Myanmar, a Southeast Asian country bordering Bangladesh, India, China, Laos, and Thailand. Until recently, the country had been under military rule since 1962, and became, "one of the 
world's least developed and least free countries" (Ranard \& Barron, 2007, p. 1). There are many different ethnic groups from Burma, several of whom have resettled as refugees in the United States. The largest ethnic refugee group is the Burmans (Ranard \& Barron, 2007), but others include Karen, Karenni, Rohingya, Shan, and Chin. For the purposes of this paper, due to their similar experiences of being refugees resettled to the United States, I am grouping together all of the Burmese ethnic groups rather than treating them individually. The different groups all came from similar, sometimes the same, refugee camps in countries that neighbor Burma. They have been placed together in the United States, and the students typically associate with each other regardless of their specific ethnic groups.

Identity. In middle school, identity is a major part of one's development. According to McLean et al., (2010), “Adolescence is a time when identity development takes center stage” (p. 184). Habermas and Bluck (2000) and McAdams (2001) agreed that the identity formation process begins in adolescence. Many theories describe and explain how a person's sense of identity forms. Bucholtz and Hall (2005) used the grounding of sociocultural theory to propose a model for identity formation. In their model, identity is "the social positioning of self and other" (Bucholtz \& Hall, 2005, p. 586), so it is through social interaction that identities are formed. Bucholtz and Hall (2005) stated, "Identity is a discursive construct that emerges in interaction" (p. 587). Their theory encompasses five principles: emergence (identity is emergent from interaction), positionality (identity emerges from temporary interactional positions), indexicality (identities are shaped through shared understanding of labels and 
assumptions attached to those labels), relationality (identity is based on several overlapping relationships), and partialness (views of identity are always partial due to the complexity of identity).

Similar to the sociocultural theory of identity formation, Gee (2000) outlined a model in which identity is formed via interactions with others. Gee described four different perspectives on identity: the nature perspective, the institutional perspective, the discursive perspective, and the affinity perspective. In his discursive perspective, people's identities are ascribed by how other people view, treat, and interact with them (Gee, 2000). Neither Bucholtz and Hall (2005) nor Gee, however, described how the identities are actually formed. Sfard and Prusak (2005) offered a way to examine identity formation by viewing them as stories.

Sfard and Prusak (2005) defined identity as a story about a person told by someone to someone. The storyteller and the recipient can change; either can be the identified person or another person. The four different ways of identifying a person are: (a) a story told by the identified to another person, (b) a story told by another person to the identified, (c) a story told by another person about the identified to yet another person, or (d) a story told by the identified to him or herself. For three of these possibilities, interaction with others is central.

Mosselson (2006) presented a framework specific to refugee identity construction called "roots \& routes" (p. 20). In this model, refugees simultaneously looked back on their histories and forward to their future as they constructed their identities. In this way, their identities were constantly in flux and never fully settled. According to these models 
taken together, identities are stories people tell about themselves that is ever changing based on their experiences and their ideas of who they want to be in the future.

Identity formation within one person is not monolithic. Researchers have acknowledged that people have different identities in different situations. Bucholtz and Hall (2005) asserted that identities are based on relationships, meaning one's identity could be influenced depending on the other person involved. Similarly, Esteban-Guitart and Moll (2014a; 2014b) examined identity through a Vygotskian lens, concluding that identities are formed through an active, ever-changing process based on multiple inputs throughout a person's life. As Gee (2000) noted people's identities can (a) change from moment to moment (b) can be in different contexts, or (c) be ambiguous. According to Sfard and Prusak (2005), each story that explains one's identity could be different. Inherent in Mosselson's (2006) framework is the flexible nature of refugee's identities in relation to different experiences.

Hegemony. According to McLaren (2009), "Hegemony refers to the maintenance of domination not by the sheer exercise of force but primarily through consensual social practices, social forms, and social structures produced in specific sites such as the church, the state, the school, the mass media, the political system, and the family" (p. 67). I am using hegemonic culture distinctly from dominant culture because hegemonic norms are not overtly, or dominantly, expressed. Because norms are not dominantly expressed, the oppressed are "unknowingly participating in their own oppression" (McLaren, 2009, p. 67). I am also using hegemonic culture in place of the term many critical race theorists use: white supremacy (Cole \& Maisuria, 2007: 
Leonardo, 2009; Taylor, 2009). Cole and Maisuria (2007) pointed out that the term white supremacy homogenizes all people who are white, even though many white people are not in power. According to Leonardo (2004), power is an important part of any racial discussion. Though I acknowledge that the dominant culture of white people, white power is a much more nuanced term than is used by the general public (Gillborn, 2010), and that many white people — though not all (Cole, 2009)—benefit from simply being white, whether or not they are part of the power structure (Ladson-Billings, 2009; McIntosh, 1988), I prefer the term hegemonic culture because it connotes a normative power structure in addition to race. 
BURMESE REFUGEE STUDENT INTERACTION

\section{Chapter 2: Review of the Literature}

In the previous chapter, I explained the need to study how female Burmese refugee students interact with students from other cultural backgrounds. Burmese refugee students are part of a large and relatively new group who has tended not to interact with students from other cultural backgrounds in my school. In this chapter, I review the literature about various aspects of the problem. After specifying my theoretical framework (Bourdieu's theory of field and theory of social and cultural reproduction) for my problem of practice, I report what researchers have found about child development for middle school students, multicultural education, intercultural interactions, refugee education, female refugees, and Burmese refugee students. I also outline the methods I used to conduct my study.

\section{Theoretical Framework}

Burmese refugee students enter schools in the United States with many challenges that can hinder their success in school (Dávila, 2012; Dryden-Peterson, 2015; Gitlin et al., 2003; McBrien, 2005; Portes \& Zhou, 1993; Pugh et al., 2012; Valenta, 2010). One way of overcoming their challenges may be intercultural interactions (Gurin et al., 2011; Phinney et al., 2007; Ryan \& Patrick, 2001). To frame how Burmese refugee students interact with students from other cultural backgrounds and how intercultural interactions help them overcome their challenges, I use Bourdieu's theory of field and theory of social and cultural reproduction (Bourdieu, 1973, 1977, 1990; Bourdieu \& Wacquant, 1992), which posits that people bring their collective experiences into fields shaped by the actions and attitudes by those in the field. The norms of the field, in turn, shape the 
actions of the people in the field. Those whose actions most closely match the norms of a particular field gain more power to shape the field in which they operate.

Nolan (2012) summed up Bourdieu's theory of field in the following manner: According to Bourdieu, everyday decisions are made within a network of structures and relations, referred to as a field. Those decisions shape, and are shaped by, a set of dispositions (habitus) that include attitudes, beliefs, perceptions, and practices, all formed through the embodiment of one's life history. (p. 204)

In other words, the fields, or contexts in which people operate, affect their actions. At the same time, the field is shaped by people's collective actions. An important part of Bourdieu's theory of field is that people whose actions most closely match the norms of the field have cultural capital, or valued interests, skills, and behaviors (Bourdieu \& Wacquant, 1992). The concept of cultural capital is central to another of Bourdieu's theories that I use for my theoretical framework: the theory of social and cultural reproduction (Bourdieu, 1973). In this theory, schools are a key place for the transmission of cultural capital from one generation to another. Adults teach acceptable cultural norms to students, reproducing ways of behaving that grant access to privilege.

The theory of field and the theory of social and cultural reproduction overlap and are referred to by multiple names. Nolan (2012) referred to the former as social field theory, while Vorhaus (2014) referred to the latter as social capital theory. Other scholars (e.g., Albert \& Kleinman, 2011; Peters, 2014) have discussed Bourdieu's theories holistically, without referring to the theory of field or the theory of social and cultural 
reproduction. Greenspan (2014) also discussed the theories holistically, but called them Bourdieu's theory of capital. For my theoretical framework, I treat the theories as related, but discuss them separately because of their slightly different foci. The theory of field is descriptive of existing power structures within a field, whereas the theory of social and cultural reproduction is descriptive of how those power structures are transmitted. In my study, I did not have the luxury of investing enough time to see cultural capital passed from one generation to the next, but it is important to take into account how norms may change over time based on specific dispositions of actors in the field. With that in mind, I describe the key points of Bourdieu's theory of field and theory of social and cultural reproduction.

\section{Theory of Field}

In his theory of field, Bourdieu (1977) stated that cultural power structures result in norms of behavior within a given field. Depending on how closely they match the accepted behavioral norms, individuals' behaviors are assigned value by the rest of the field. Individuals are more successful when their behaviors align with the accepted norms and less successful when they do not. Social norms are reproduced via people tailoring their actions, behaviors, and beliefs toward the accepted norms in a particular

field. Bourdieu and Wacquant (1992) defined field as:

A network, or a configuration, of objective relations between positions. These positions are objectively defined, in their existence and in the determinations they impose upon their occupants, agents or institutions, by their present and potential situation (situs) in the structure of the distribution of species of power (or capital) 
whose possession commands access to the specific profits that are at stake in the field, as well as by their objective relation to other positions (domination, subordination, homology, etc.). (p. 97)

Though Bourdieu and Wacquant were discussing economics in the above quote, the ideas they put forth also apply to education in that fields are contexts into which people bring dispositions such as attitudes, habits, beliefs, and perceptions that have been formed by their past experiences. According to Albert and Kleinman (2011), fields are arenas in which people struggle for power to have their own practices viewed as legitimate by others in the field. Legitimacy comes from criteria set by experts in the field, but can be changed by those with legitimacy. People with more legitimacy have more power with which to set the criteria according to which others will be judged. Albert and Kleinman stated, "The key aspects of Bourdieu's concept of field is that it highlights the importance of the symbolic, or cultural, dimension of the struggle for power" (p. 266).

Those who have power differ from field to field because the norms of each field are distinct. According to Bourdieu and Wacquant (1992):

Each field calls forth and gives life to a specific form of interest, a specific illusio, as tacit recognition of the value of the stakes of the game and as practical mastery of its rules. Furthermore, this specific interest implied by one's participation in the game differentiates itself according to the position occupied in the game (dominant vs. dominated or orthodox vs. heretic) and with the trajectory that leads each participant to this position. (p. 117) 
People bring in their own interests into each field, which are in turn shaped by the field. Bourdieu and Wacquant (1992) explained, though, that this does not happen in a circular fashion; rather, it is multilayered. Each field is formed over time by multiple people's competition for power; people bring into a field their multifaceted histories and interests. All parts affect each other over time.

Bourdieu (1977) referred to the interests and dispositions that people bring into a field as habitus, which he defined as:

Systems of durable, transposable dispositions, structured structures predisposed to function as structuring structures, that is, as principles of the generation and structuring of practices and representations which can be objectively 'regulated' and 'regular' without in any way being the product of obedience to rules, objectively adapted to their goals without presupposing a conscious aiming at ends or an express mastery of the operations necessary to attain them and, being all this, collectively orchestrated without being the product of the orchestrating action of a conductor. (p. 72)

By dispositions, Bourdieu (1977) means the overall attitudes, habits, or tendencies one has within a particular field. Taken another way, "to speak of habitus is to assert that the individual, and even the personal, the subjective, is social, collective. Habitus is a socialized subjectivity" (Bourdieu \& Wacquant, 1992, p. 126). In other words, habitus is the internalization of societal expectations by an individual as he or she is exposed to cultural norms. 
BURMESE REFUGEE STUDENT INTERACTION

The closer a person's inherent dispositions are to the accepted dispositions of the field, the more likely it is the person will be successful in that field (Bourdieu, 1990). The field does not create one's habitus, nor do collective habitus create a field. According to Bourdieu and Wacquant (1992):

The relation between habitus and field operates in two ways. On one side, it is a relation of conditioning: the field structures the habitus, which is the product of the embodiment of the immanent necessity of a field (or of a set of intersecting fields, the extent of their intersection of discrepancy being at the root of a divided or even torn habitus). On the other side it is a relation of knowledge or cognitive construction. Habitus contributes to constituting the field as a meaningful world, a world endowed with sense and value, in which it is worth investing one's energy. Two things follow. First, the relation of knowledge depends on the relation of conditioning that precedes it and fashions the structures of habitus. Second, social science is necessarily a 'knowledge of a knowledge' and must make room for a sociologically grounded phenomenology of the primary experience of the field or, to be more precise, of the invariant and variation of the relation between different types of fields and different types of habitus. (p. 127) People are socialized to dispositions that are successful in a given field. At the same time, certain habitus within a field give the field meaning, which perpetuate certain habitus. Bourdieu (1990) said this results in, “Agents whose dispositions are the product of conditionings associated with positively distinguished positions... have only to give way to their dispositions to produce practices that are 'naturally' distinguished" (p. 108- 
109). People who grow up in positions of power within certain fields have habitus that match the desired dispositions within those fields. They seem to naturally understand how to navigate those fields. By extension, people who do not grow up in positions of power within a field and do not have ingrained understandings of how to be successful in that field are at a disadvantage.

In sum, people with collective dispositions come together in fields, which are arenas in which people struggle for power (Albert \& Kleinman, 2011). Within each field some habitus are more accepted than others. Those exhibiting habitus that are more accepted have more capital, and therefore have more access to benefits within the field. One's position in relation to accepted habitus determines his or her access to capital, meaning that certain habitus are more valuable than others. The most valuable habitus become the norm for the group. People who can recreate the most accepted norms become the most successful; those who cannot recreate those norms are marginalized (Nolan, 2012).

If matching one's habitus to the successful dispositions necessary for success in a field were the way to achieve success in a field, a person would only have to behave as if he or she had the desired habitus; he or she would not have to internalize successful dispositions. However, fields are not static. According to Bourdieu and Wacquant (1992):

Social collectives such as bureaucracies have built-in propensities to perpetuate their being, something akin to a memory or a loyalty that is nothing other than the 'sum' of routines and conducts of agents who, relying on their know-how 
BURMESE REFUGEE STUDENT INTERACTION

(métier), their habitus, engender (within the limits of the constraints inscribed in the relations of force constitutive of the field of which they partake and the struggles which oppose them) lines of action adapted to the situation such as their habitus inclines them to perceive it, thus tailor made (without being designed as such) to reproduce the structure of which their habitus is the product. (p. 139140)

Participants in a field continually reshape the field such that their habitus are more successful. Over time, the field, and the successful habitus can change quite drastically. In addition, habitus that are ill-fitted to the field can persist out of inertia (Bourdieu \& Wacquant, 1992). Those who are not familiar with the specific habitus that are successful in a particular field are at a disadvantage when it comes to being successful. Because habitus can change as fields change over time, one cannot simply learn the most successful dispositions and become successful. This is especially important for marginalized populations who do not have the opportunity to have extensive experience learning the most successful dispositions. Because the field is ever-changing, marginalized populations continually play catch-up in internalizing successful dispositions.

Bourdieu's two theories overlap and are often treated together in the research (Albert \& Kleinman, 2011; Greenspan, 2014; Peters, 2014). For the purposes of this paper, I discuss them separately. Next, I explain Bourdieu's (1973) theory of social and cultural reproduction. 
BURMESE REFUGEE STUDENT INTERACTION

\section{Theory of Social and Cultural Reproduction}

Bourdieu and Wacquant (1992) referred to six different types of capital: cultural, economic, juridical, scientific, social, and symbolic. Greenspan (2014) claimed Bourdieu distinguished between three fundamental types of capital; cultural, economic, and social. Cultural capital refers to educational qualifications or "value in long-lasting dispositions, such as ethnicity, religion, or family background, acquired from early childhood or through socialization" (Greenspan, 2014, p. 103). Economic capital refers to traits that can be converted into money. Social capital refers to the capital that accrues over time through mutually recognized institutionalized relationships (Bourdieu \& Wacquant, 1992). The time component in social capital speaks to how capital can be transmitted from one generation to the next. According to Bourdieu's (1977) theory of field, people gain capital when their habitus match the accepted norms of the field.

According to Bourdieu (1973), the educational system is explicitly tasked with transmitting cultural capital to students. Because traits of the dominant classes carry more value in society, those traits are emphasized by the system and treated as normal. To be successful in school, students must closely match the habitus of the dominant society as transmitted through the educational system.

Bourdieu's two theories accurately describe some of the challenges Burmese students face after entering the United States. To gain access to their particular field (e.g., a middle school classroom), and be successful in that field, they have to learn the accepted norms, which might be different from those of their home culture. Though Burmese refugees come from many different cultural groups, most of my students have 
tended to be shyer and quieter than their peers, which may be indicative of their cultural emphasis on community and respect for others (Ranard \& Barron, 2007). If a cultural norm in classrooms in the United States is to advocate for oneself, Burmese refugee students' shyness could keep them from being successful.

Because the norms can change over time based on the behaviors and attitudes of students with more cultural capital, simply learning that becoming more outspoken will result in success is not a guarantee that Burmese refugee students will become successful. They must more deeply internalize the specific habitus within the school to gain cultural capital and be successful. Because schools are such complex fields (Bourdieu, 1990), navigating them without internalizing the successful habitus is especially difficult.

\section{Critique of Bourdieu's Theories}

Though Bourdieu's (1973, 1977, 1990; Bourdieu \& Wacquant, 1992) theories of field and social and cultural reproduction accurately capture the experiences Burmese students have in my classroom, some issues remain with its application. First, to gain cultural capital in Bourdieu's theories, students must internalize the habitus dominant in the particular field. This places the impetus for change upon those marginalized within the field. Coupled with the assertion by Tzanakis (2011) that schools do not blindly transmit cultural capital, Bourdieu's theories seem to ask a lot of marginalized groups to gain capital and be successful.

Bourdieu (1973, 1977, 1990; Bourdieu \& Wacquant, 1992) wrote descriptively, so he was not attempting to advocate a particular view of how marginalized students should gain capital. According to Tzanakis (2011), Bourdieu emphasized class-based 
differences that do not seem to be a major factor when it comes to ethnic minorities, such as Burmese refugee students. Olneck (2000) stated that multicultural education could be an effective way for ethnic minority students to shape cultural capital. Put another way, students help form the habitus in particular classrooms. If the teacher can ensure all students have input into the accepted habitus, marginalized students could help shape the norms and automatically gain power.

Though Bourdieu (1973, 1977, 1990; Bourdieu \& Wacquant, 1992) never explicitly stated that each field carried only one set of norms, the implication in his theories is that there exists one set of dominant norms within each field. In heterogeneous contexts, it seems that more than one set of norms could exist. If more than one set of norms exists, marginalized groups might have an easier time gaining capital than if only one set of norms exists.

Another critique of Bourdieu's theories came from Yosso (2005), who examined the concepts from the lens of critical race theory (CRT). Yosso contended that Bourdieu's description of capital seemed to place a deficit view upon marginalized people. Yet, when viewed through the lens of CRT, marginalized people bring their own types of capital into fields. Yosso delineated six types of capital including (a) aspirational, (b) linguistic, (c) familial, (d) social, (e) navigation, and (f) resistant. She asserted that marginalized students are able to shape the norms in a classroom through the capital they bring with them. 


\section{Summary of Theoretical Framework}

In a multicultural middle school such as mine, students from a variety of different cultural backgrounds come together in fields called classrooms. Within those fields, some dispositions and behaviors (habitus) are more collectively valued than others. Students whose habitus most closely match the valued habitus gain more cultural capital than others. The cultural capital that makes students successful is transmitted through schools. For marginalized students to gain cultural capital and be successful in a classroom, they must learn and internalize the accepted habitus. Classrooms, though, do not form habitus absent the input of the students in the class. Students with more cultural capital have greater influence over the accepted habitus. Over time, habitus changes because of the influence of the students. As marginalized students help to form the norms of a classroom, their habitus would more closely match those norms, and, thus, the marginalized students would gain cultural capital.

Viewing my topic through the lens of Bourdieu's theory of field, I next review the literature regarding child development for middle school students, multicultural education, intercultural interactions, refugee education, female refugees, and Burmese refugee students.

\section{Review of the Research Literature}

Using Bourdieu's framework, when Burmese students interact with students from other cultural backgrounds in middle school, they learn successful habitus in the field of the classroom and gain access to cultural capital. To further explore this idea, I first review the literature regarding the setting in which intercultural interactions in my 
multicultural middle school occur. Then, I review what researchers have found regarding intercultural interactions. Because I examined the experiences of female Burmese refugees in intercultural interactions, I report what is known about refugee education and female refugee education before focusing more narrowly on Burmese refugees.

\section{Middle School and Identity Formation}

For young adolescents in middle school, peer interaction is especially important. Peer interaction is a crucial part of the identity formation of young adolescents, which takes center stage during middle school. According to Para (2008), peers offer support when family cannot. Often, young adolescents seek peer approval and have a strong focus on group belonging as they reorient from their family toward their peers (Caskey \& Anfara, 2014).

Undergirding much of the literature on adolescent development is the work of Erikson $(1950,1968)$. He theorized that people form their identities in stages from birth to late adulthood. At each stage, an individual has a social conflict to navigate. In infancy, the conflict is whether or not the individual can trust others. In early childhood, people struggle with whether or not they can be autonomous and develop a sense of free will. From roughly ages three to five, children try to form initiative. When children enter school and until early adolescence, they navigate whether or not they are industrious enough to create things. In adolescence, children are concerned with their senses of identity and how others may see them. After adolescence, adults become concerned with partnering with another person, whether or not they continue to grow and be useful to society, and if their lives carried meaning. 
In middle school, students are firmly within the stage Erikson (1950) called identity versus role confusion. According to Erikson, adolescents continue to navigate the earlier conflicts of trust versus mistrust, autonomy versus shame, initiative versus guilt, and industry versus inferiority. In the identity versus role confusion stage, adolescents are preoccupied with finding the person they will become. The danger in not doing so is experiencing role confusion. One of the reactions typical of adolescents is to form cliques as a defense against not finding an identity and a test of loyalty. Erikson emphasized that adolescents are at an ideological stage moving toward adulthood. In this stage, they are searching for confirmation that good will win out over bad.

According to Erikson (1950), while forming their identities in adolescence, children will often stress loyalty toward people and/or ideas while also protecting themselves against being perceived as foolish by acting cynical. In addition, they tend to stress independence, but still be willing to follow adult directions because they would rather be seen as foolish because of an adult than be seen as choosing to be foolish. In wanting to find their permanent identities, adolescents sometimes refuse to work on tasks they do not see as useful for their future.

Because of their dual emphases on independence and self-protection, one might see adolescent behavior as inconsistent and moody. Smetana, Campione-Barr, and Metzger (2006) stated that the popular view of adolescence (which they defined as being roughly between ages 10 and early 20s) has been a period during which people are typically moody and rebel from their parents. 
While many adolescents seem to grow away from parents and toward their peers, several studies indicate a more complex situation. Bowerman and Kinch (1959) used questionnaires to find how 686 white, middle-class students from two-parent families north of Seattle, Washington oriented themselves toward family or peers. The students were roughly distributed from fourth through tenth grades, with four core classrooms from each of the seven grades filling out the questionnaires. From 4th to 10th grade, students shifted from predominantly family-oriented to predominantly neutral and peeroriented. Bowerman and Kinch attributed this shift to increases in peer-related activities through adolescence and maladjustment by the family toward the adolescent's changes. If a family did not adjust to a child's increasing orientation toward peers, the child decreased his or her orientation toward the family. They acknowledged that they measured only predominant orientation rather than degrees of orientation that may have provided better explanations for the phenomenon of increased peer orientation. Notably, this study only measured a narrow band of students that attend public schools (white, middle-class students with two parents).

Berndt (1979) conducted two studies to examine the change in adolescent conformity from parents to peers in antisocial, prosocial, and neutral behaviors. Berndt's first study involved 251 children roughly equally distributed between third, sixth, ninth, and $11^{\text {th }}$ and $12^{\text {th }}$ grades $\left(11^{\text {th }}\right.$ and $12^{\text {th }}$ grades were mixed). More than $90 \%$ of the participants were Caucasian and all were from a middle- and working-class city. The students answered questionnaires evaluating antisocial actions and responding to various peer-suggested situations. The situations were evenly split among antisocial, neutral, and 
prosocial behaviors. For all neutral and antisocial situations, student conformity to peers increased from third to ninth grade, then decreased in $11^{\text {th }}$ and $12^{\text {th }}$ grade. For prosocial situations, conformity increased from third to sixth grade, then decreased. Berndt concluded that conformity to peers peaked during mid-adolescence.

Berndt's (1979) second study focused on the change in conformity from parents to peers in prosocial and neutral situations. In this study, 273 students participated, again roughly evenly distributed between third, sixth, ninth, and $11^{\text {th }}$ and $12^{\text {th }}$ grades. On questionnaires, participants evaluated antisocial situations and their responses to suggestions from either peers or parents. Parent conformity decreased for both prosocial and neutral situations, but only by a small amount. Peer conformity for prosocial behaviors decreased from third to sixth grades, increased in ninth grade, then decreased again in $11^{\text {th }}$ and $12^{\text {th }}$ grades. Peer conformity for antisocial behaviors increased from third to ninth grade, then decreased in $11^{\text {th }}$ and $12^{\text {th }}$ grade. Overall, these two studies indicate that while parent influence somewhat decreased throughout adolescence, peer influence increased to a peak in ninth grade. Like the Bowerman and Kinch (1959) study however, Berndt only studied a small range of school-age students (white, middle-class). Smetana (1988) also looked at how adolescents shift their orientation from adults to peers in examining adolescents' and parents' conceptions of the legitimacy of parental authority regarding acts in different conceptual domains. Smetana studied 102 children from fifth grade to 12 th grade, split into four clusters of two grades each. The students came from middle to upper middle class, Caucasian families with most parents having college degrees. Smetana presented each family with 15 transgressions written on cards. 
Participants first sorted the cards into two categories according to whether or not it was okay for parents to make rules, then justified their categorization, and finally recategorized the cards into whether the act was always wrong, wrong if the parent said so, or never wrong. Answers were given numerical values and statistically analyzed. The results indicated that as children move into adolescence, they viewed multifaceted and personal issues as less affected by parental authority than moral or conventional issues. Smetana concluded that adolescents grow increasingly independent of their parents in some domains, but not unilaterally. Like the previous studies, though, the participants were not representative of the population where my study took place.

Larson and Richards (1991) also found that adolescents do not unilaterally reorient themselves from parents to peers. They studied how much time adolescents spend with family and friends and how they felt about their time spent with both. Participants were 483 fifth to ninth grades from predominantly white working, middle, and upper class neighborhoods in suburban Chicago. Each participant wore a pager that sent a signal at seven random points throughout the day. When the pager sent the signal, the participant filled out a self-report form stating what they were doing and how they felt about it. Responses were assigned numerical values and statistically analyzed. Larson and Richards concluded that as children move through adolescence, their time with family decreases, though time alone with only mother and father (without siblings) did not decrease. In addition, older adolescents did not report less happiness when with family than younger children. Adolescents spent only slightly more time with friends, but reported increasingly positive feelings toward friends. Time alone increased even 
though it was associated with a less positive affect. Again, this research was conducted with a relatively homogeneous population unrepresentative of the participants in my study.

Steinberg and Silverberg (1986) studied a wider range of school-age students in examining the development of autonomy among adolescents. Steinberg and Silverberg examined 865 adolescents nearly evenly distributed among grades five through nine in Madison, Wisconsin. Though predominantly white (86\%), the sample came from a range of socioeconomic backgrounds, unlike the studies by Bowerman and Kinch (1959) and Berndt (1979). Steinberg and Silverberg (1986) administered a questionnaire using a Likert-Scale to measure emotional autonomy, susceptibility to peer pressure, and sense of self-reliance. They reported that adolescents traded dependency on parents for dependency on peers. Students' views of their parents became less idealized over time. Their susceptibility to peers increased through ninth grade. Girls, overall, were more autonomous than boys at all levels. Even though Steinberg and Silverberg studied a diverse socioeconomic sample, the sample was relatively culturally homogeneous.

Based on the above studies, it seems that adolescents tend to drift away from their parents and towards their peers, but not linearly. Importantly, none of the studies examined the experiences of minority students within Erikson's (1950) identity versus role confusion stage. It is possible that the experiences of minority adolescents could be quite different than those of adolescents from the majority culture.

Ethnic identity formation. The idea that adolescents from a minority ethnic background might have different experiences than adolescents from the dominant culture 
is not new. Erikson (1968) addressed the possibility in the 1960s. A few researchers have focused their studies on the development of ethnic identity among adolescents and have helped to shed light on the experiences of minority populations. Much of the research has grown from Marcia's (1980) work operationalizing Erikson's $(1950,1968)$ identity versus inferiority stage. Marcia (1980) stressed that identity formation does not begin in adolescence, but adolescence is a particularly important time for this process. In his review, Marcia concluded that identity is a measurable construct with utility in personality development. According to Phinney (1989), Marcia's work $(1966,1980)$ has led to others' work on ethnic identity.

Marcia (1966) claimed identity for ethnic minorities falls into one of four statuses based on exploration and commitment: diffuse, foreclosed, moratorium, or achieved (see Table 1). A person has a diffuse identity when he or she have not engaged in nor committed to an identity. A person has a foreclosed identity when he or she has settled on an identity without exploration. A person has a moratorium identity when he or she is exploring without having made a commitment. A person has an achieved identity when he or she has explored possible identities and committed to one. Several researchers have used Marcia's framework to study ethnic identity formation in adolescence. 
BURMESE REFUGEE STUDENT INTERACTION

Table 1

Ethnic Identity Formation - Marcia (1980)

\begin{tabular}{lllll}
\hline Status & Diffuse & Foreclosed & Moratorium & Achieved \\
\hline Description & No exploration & No exploration & Exploration & Exploration \\
& No commitment & Commitment & $\begin{array}{l}\text { No } \\
\text { commitment }\end{array}$ & Commitment \\
& & & & \\
\hline
\end{tabular}

Phinney (1988) surveyed 300 undergraduates of various ethnic backgrounds at a multicultural university. She came to six conclusions: (a) ethnic identity was important for all groups, (b) students of mixed ethnic backgrounds experience more identity conflict than other groups, (c) most minority students were deeply exploring their identities by late adolescence, (d) minority students began their identity formation process a an earlier age than students from the majority culture, (e) for minority students there is a strong relationship between ethnic identity and self-esteem, and (f) students from minority ethnic backgrounds search more than, but have similar levels of commitment as, students from the majority. Though Phinney's study examined the experiences of older adolescents who had already gone through most of their ethnic identity formation, it makes clear that ethnic identity formation is an important part of adolescent development, and identity formation happens slightly differently for ethnic minorities than for adolescents from the hegemonic culture.

In a different study of 91 Asian-American, Black, Hispanic, and White $10^{\text {th }}$ grade American-born students in multicultural urban high schools, Phinney (1989) focused more on ethnic identity formation. Participants were interviewed about their ethnicity 
and given questionnaires measuring their ego identity. Three graduate students coded the interviews and questionnaires according to ethnic identity stages. Phinney found that the responses of White participants could not be coded. Of the 64 minority participants, 60 were reliably coded as initial (equivalent to Marcia's [1966] diffuse and foreclosure stages), moratorium, or achieved. Almost $56 \%$ of participants were in the initial stage, about $23 \%$ were in the moratorium stage, and just over $21 \%$ were in the achieved identity stage. Phinney reported no significant differences in the stages between Asian American, Black, or Hispanic students; nor did differences exist between genders or classes. Some interesting differences were revealed in the individual responses. More Asian Americans said they would prefer to be White than did Black or Hispanic students. Salient issues were also different for each minority group. Asian Americans were concerned with academic issues; Black females were concerned with standards of beauty; Black males were concerned with job discrimination and negative issues of Black adolescents; Hispanic students were concerned with prejudice and conflicting values between home and dominant society. Phinney (1989) concluded that this study provided empirical evidence for a version of Marcia's (1966) identity stages as they apply to ethnic identity across ethnic backgrounds. Coders were not able to distinguish between the two earliest stages (diffuse and foreclosure), but Phinney (1989) found evidence for an initial stage that combines diffuse and foreclosure, a middle stage called moratorium, and a final stage called achieved.

To test developmental models of ethnic identity development like Marcia's (1966), French, Seidman, Allen, and Aber (2006) conducted a three-year-long 
longitudinal study with 420 African American, Latino American, and European American young and middle adolescents. Their main focus was to examine how ethnic identity develops over time. Secondary foci included comparing ethnic identity across ethnic groups, assessing whether there was increased group-esteem and exploration through adolescence, and examining whether the transition from junior to high school resulted in different patterns of change than adolescents spending two years in the same school. French et al. found that for students to reach achieved identities, they must engage in exploration. For students to engage in exploration, though, they may need to experience heterogeneous contexts. In heterogeneous environments, incidents of racial prejudice increase, which trigger increases in exploration. According to French et al., subethnic groups have similar enough experiences to be discussed with their larger ethnic groups, though they recommend researchers continue to study different subethnic groups. The three ethnic groups examined by French et al. exhibited different amounts of change in self-esteem over the three years; European Americans began high and stayed high, African Americans and Latino Americans began low and dramatically increased. French et al. noted that adolescents across ethnic groups engage in explorations of ethnic identity and delineated some similarities and differences between groups, Asian Americans (including Burmese students) were conspicuously absent. Despite the absence of Asian Americans, the researchers suggested a multicultural context, such as the setting of the study reported in this paper, should be beneficial for students' exploration, and subsequently their ethnic identity formation. 
Gartner, Kiang, and Supple (2014) specifically examined ethnic socialization (what adults share about their children's home cultures and how to interact with people from other cultures) and ethnic identities of 147 Asian American ninth and $10^{\text {th }}$ grader students over more than three years. All data were gathered in the form of questionnaires. Based on their analysis of the questionnaire data, Gartner et al. reported that Asian and American identity formations were not mutually exclusive; both could develop simultaneously in adolescents. Additionally, they noted that ethnic socialization preceded advancement through levels of ethnic identity, which leads to higher levels of self-esteem. While French et al. (2006) suggested continued study with both subethnic and specific ethnic groups, and Gartner et al. studied Asian Americans, Burmese refugees were still absent from the literature regarding adolescent identity formation.

Esteban-Guitart and Moll (2014a, 2014b) considered identity from a Vygotskian perspective wherein people's cultural identities are formed through their interactions with the outside world. As a complicated, active, ever-changing process, identity forms through the many inputs over the course of people's lives. It is a lived experience in which "a person perceives and attributes values, senses and meanings to any funds of identity" (Esteban-Guitart \& Moll, 2014b, p. 75). In this view of identity, the knowledge, backgrounds, and experiences of the students are valued as resources for learning. According to Hviid and Villadsen (2014), educators could benefit from understanding children's identities as processes that form through interactions with others and create inherent value within the students. 
Though still not addressing Burmese refugees, Mosselson (2006) presented a framework specific to refugee identity formation. In the study, Mosselson interviewed fifteen female Bosnian refugees between the ages of 15 and 24. In addition, Mosselson interviewed 10 other members of the Bosnian refugee community, "in order to triangulate and contextualize the core participants' self-narratives" (p. 21). A main conclusion from the study was a framework called "roots \& routes" (p. 20). In the Mosselson framework, refugees form their identities through their descriptions of their places of origin (roots) and how they visualize their futures in light of their positions in their communities in diaspora (routes). Mosselson's framework allows for viewing refugee identity formation in light of their communities rather than solely within the individual. In addition, this framework treated identity formation as fluid and ever-changing. According to Mosselson, the fluid nature of roots \& routes helped the participants cope with various struggles in their lives.

While some theorists of ethnic identity formation have framed identity as a singular concept within a person, many researchers (e.g., Bucholtz and Hall, 2005; Esteban-Guitart \& Moll, 2014a, 2014b; Gee, 2000; Mosselson, 2006) viewed identity formation as multi-faceted and ever-changing.

In sum, according to Erikson's $(1950,1968)$ framework, adolescents navigate forming their identities and finding their places in the world. They tend protect themselves against embarrassment (Erikson, 1950) and gravitate toward their peers (Larson \& Richards, 1991; Smetana et al., 2006). As early as 1968, Erickson (1968) suggested the possibility that adolescents from minority ethnic backgrounds may 
experience a different developmental process than those from the majority ethnic

population. Largely based on the work of Marcia (1966, 1980), several researchers have focused on identity formation among ethnic minority adolescents. Students from ethnic minorities appear to form identities differently than do adolescents from the majority population (Phinney, 1988) by going through stages similar to Marcia's (1966) framework (French et al., 2006; Phinney, 1989). For refugees in particular, their identity formation process is fluid as it involves constantly looking back toward the past and forward toward the future (Mosselson, 2006). For exploration toward a secure ethnic identity to occur, it appears a multicultural context is beneficial (French et al., 2006). Though researchers have studied the ethnic identity development of several minority adolescent groups, Asian refugees, including Burmese, have not been examined. Because interaction is central to identity formation, the identity formation process begins in middle school, and ethnic socialization precedes ethnic identity formation in Asian Americans, it stands to reason that intercultural interaction is a central part of early adolescence for minority groups such as Burmese refugees. In the next section, I examine the multicultural context in which students can engage in intercultural interactions and form ethnic identities in middle school.

\section{Multicultural Context}

I conducted my study in a school with nearly 900 students who come from more than 35 cultures from all over the world. In any given class, there might be students who speak up to 20 different languages. In such a context, students have ample opportunities 
to interact with others from a variety of cultural backgrounds. Such a setting is what Vertovec (2007) terms super-diverse.

Though Vertovec (2007) focused on immigration patterns in London since the early 1990s, his conclusions about the effects of super-diversity are applicable elsewhere. He argues for a complex view of multiculturalism in settings with a variety of different immigrant groups. Each group comes with their own experiences and backgrounds, which means researchers and policy-makers must account for a range of different intersecting variables. The variables include country of origin, migration channel, legal status, social and cultural capital, access to employment, locality, transnationalism, and how the power structure supports (or does not support) the particular immigrant group. Though Vertovec did not conclude by giving specific suggestions, primarily because each super-diverse context is different depending upon the groups that constitute the context, the framework he provided is useful to consider in such a multicultural setting.

In multicultural settings, it stands to reason that teachers need to emphasize multicultural education. The major idea behind multicultural education in a diverse setting is to provide equal opportunities for all students to be successful. According to Lee (2003):

Students from diverse languages and cultures come to school with previously constructed knowledge, including their home language and cultural values, from home and community environments. When these students are provided with equitable learning opportunities, they can capitalize on their linguistic and cultural experiences as intellectual resources for new learning. (p. 466) 
In other words, educators need to provide opportunities for students from non-dominant cultures to access the benefits of the hegemonic culture. Through the process of multicultural education, students gain access to Bourdieu's (1973) idea of cultural capital, allowing them to better navigate in an unfamiliar culture. According to Bourdieu (1973), schools reproduce dominant cultural traits through generations. The cultural traits that make up cultural capital are defined and passed on by people who have the most access. People without access to or specific knowledge of cultural capital are marginalized. Though Tzanakis (2011) argued that schools do not blindly transmit cultural capital, he acknowledged it is a powerful heuristic that can lead to exclusion of minority populations.

Simply teaching the aspects of cultural capital to students, however, does not automatically result in equity for all. In examining how students acquire cultural capital, Olneck (2000) said that, though it may not be very effective at transforming cultural capital that has already formed, multicultural education can help to keep it from forming for majority students. If cultural capital is more distributed throughout ethnic groups in a multicultural setting, marginalized students will have more access to learning successful habitus and, in turn, gain cultural capital. If multicultural education can be an effective way to help marginalized populations be successful, it is important to understand multicultural education actually and how it applies to specific classrooms. Several theorists (Banks, 1993; Bennett, 2001; Daoud, 2003; Gay, 2004; Sleeter \& Grant, 1987) have reviewed slightly different tenets of multicultural education. Others (Banks, 2008; 
Gay, 2009; Lee, 2003; Malsbary, 2014; Martínez, 2010; Paris, 2009) have explored how multicultural education can be implemented in classrooms.

Multicultural education is not an end product with a formula to achieve. According to Gay (2004), it is a process that encompasses all of education. Multicultural education cannot be inserted into an existing system without modifying the system as a whole. Banks (1993) delineated five aspects of multicultural education: (a) content integration, (b) knowledge construction, (c) prejudice reduction, (d) equity pedagogy, and (e) empowering school culture. Content integration includes information about diverse racial and ethnic groups into the curriculum. Knowledge construction deals with how mainstream views of different ethnic groups have changed over time and how to help students understand these changes. Prejudice reduction focuses on helping students reduce their prejudices. Equity pedagogy is, "a dynamic instructional process that not only focuses on the identification and use of effective instructional techniques and methods but also on the context in which they are used" (Banks \& Banks, 1995, p. 153). Last, Banks (1993) stated that schools should be restructured so that students from diverse racial, ethnic, and social-class backgrounds can achieve success. Banks acknowledged that the criteria he used have limitations, and do not necessarily capture all of the nuances of multicultural education.

Bennett (2001) provided a detailed overview of different components of multicultural education. He stated that it has four broad principles: (a) cultural pluralism, (b) social justice and the end of prejudice and discrimination, (c) affirmation of cultures in the classroom, and (d) educational equity for all. Exposure to these principles help 
teacher educators understand the nature of, think critically about, make decisions on how to implement, examine the researcher behind, and structure teacher training programs around multicultural education. Continued work in the area of multicultural education, according to Bennett, will help teachers implement, "the ideals of freedom, equality, and social justice in a culturally and racially diverse society" (p. 208).

Sleeter and Grant (1987) presented five approaches from their review of the literature: (a) teaching the culturally different, (b) human relations approach, (c) single group studies, (d) multicultural education, and (e) education that is both multicultural and social reconstructionist. They concluded that the only running theme between the five approaches was "changes in education that are supposed to benefit people of color" (p. 436). The changes were, according to their literature review, mostly implemented in the classroom. Sleeter and Grant argued that change should be more holistic rather than focused only at the classroom-level.

Daoud (2003) also recommended holistic changes to value the lives of immigrant students. Specifically, she argued the lack of interaction between native-born American students and immigrant students renders the immigrant students nearly invisible in the school fabric. Immigrant students, then, do not gain access to the cultural capital necessary for social success. Safe environments in which students can successfully interact are important, but often do not happen in mainstream content classes. One proposed solution is to emphasize a multicultural curriculum that recognizes and incorporates into the classroom the experiences of immigrant students. One could 
extrapolate that another solution might be encouraging and helping marginalized students interact more with native-born American students.

Though Sleeter and Grant (1987) argued that classroom-level implementation may not be enough and Daoud (2003) advocated holistic changes, the classroom is a key field for implementing multicultural education. Lee (2003), in a review of how culturally diverse students would benefit from a multicultural approach to science education, stressed that teachers should create learning environments where students can use their cultural backgrounds in a meaningful way. He claimed that students learn best when the content is framed in culturally meaningful ways. The job of a teacher is to connect each student's cultural experiences with the content.

Banks (2008) supported Lee's (2003) suggestion when discussing citizenship education. He asserted that citizenship education should not push assimilation, but should reflect the home cultures of students. Prior to the 1960s, Banks argued, schools emphasized nationalism. Today, this emphasis is no longer appropriate because, "These goals of citizenship education are inconsistent with the citizen's role in a global world today because many people have multiple national commitments and live in multiple nation-states" (p. 132). The emphasis needs to be on teaching students to maintain ties to their culture while exposing them to the global community. Doing so requires all cultures to be valued, especially those of students in the classroom. Because the ideas behind multicultural education are so rooted in the makeup of individual classes, much of the literature is theoretical. Studies do exist though, that examine multicultural education in context. 
Several researchers have looked more specifically at how multicultural education is employed in diverse classrooms. Malsbary (2014) examined how students develop belonging in a multiethnic and multilingual high school classroom for students learning English. She conducted three interview activities (i.e., a lifemap, a photography project, a conversational interview) with 13 students from a variety of different linguistic and ethnic backgrounds. In general, students felt a sense of belonging. They enjoyed a multilingual and transcultural atmosphere and often worked together to help each other. It was clear to Malsbary that the teacher had adjusted her approach through the course of the year to give more power over to the students. They generally felt connected as a community, though a few students felt excluded. Malsbary concluded that a multicultural classroom should pay attention to the students' goals for themselves and help them to feel like they belong in the classroom.

Paris (2009) observed and interviewed eight African American, Latino/a, and Pacific Islander focus youth and 60 other young people in their peer networks to examine their language use in their multicultural high school. Specifically, Paris looked at the use of African American Language (AAL) among the diverse groups. The use of AAL forges cohesion across ethnic groups in a marginalizing system. According to Paris, "employing the words, the grammar, and the speech acts of black language provided a space of local youth prestige against a backdrop of shared marginalization in a white language- and white culture-dominated society" (p. 443). The high school staff was generally receptive to the use of AAL among students, but did not use the grammar structure to connect students' lives to the content. While Paris conducted his study in 
high school with different minority populations than those in my study, it is important to note his insistence on using the knowledge and skills of the students in the classroom.

Martínez (2010) also conducted a study focusing on a different language pattern (a mix of Spanish and English, referred to as Spanglish), but in middle school. He observed, video and audio recorded, and interviewed students in a sixth grade language arts classroom where 28 of the 29 students were bilingual or nearly bilingual Latinas/Latinos. Like AAL in the study by Paris (2009), Martínez (2010) found that Spanglish functioned as the language of conversation for these students. He delineated six uses of Spanglish: (a) to clarify statements, (b) to quote others, (c) to joke or tease, (d) to index solidarity and intimacy, (e) to code switch, and (f) to communicate nuances of meaning. Additionally, Martínez pointed out how students' use of Spanglish paralleled the academic literacy skills expected for sixth graders. By understanding and using the skills students already possess, Martínez advocated, teachers could further students' academic growth. Though the classroom Martínez studied was not particularly diverse, the major takeaway, that students were paralleling expected skills through their use of Spanglish, echoes themes from multicultural education - schools should explicitly value and use the cultural knowledge students have when they enter the classroom.

Although studies on multicultural education are inherently specific to the context in which they occur, it is clear that when teacher students are allowed to use their strengths and create their own ways of interacting across cultures, they are connected to school, feel empowered, and grow academically. In the multicultural classrooms where I conducted my study, teachers tried to value the many different cultures of the students 
they taught by honoring their backgrounds and attempting to connect their cultural norms to the content. One way they did this was to set up situations and activities in which students could engage in intercultural interactions. In the next section, I review the literature on intercultural interactions.

\section{Intercultural Interaction}

In a multicultural context such as the school where this study takes place, students have ample opportunities to interact with students from other cultures. Intercultural interaction can benefit students' identity formation, help marginalized students learn the norms of the dominant society, and help them navigate the school system (Gurin et al., 2011; Ryan \& Patrick, 2001; Wentzel et al., 2012; Yoon, 2012). Intercultural interaction can also help students in multicultural contexts explore their identities and move through the stages of ethnic identity formation (French et al., 2006; Gartner et al., 2014). It may also help marginalized students gain cultural capital by helping to form accepted habitus in the classroom (Olneck, 2000).

As beneficial as intercultural interactions can be, they are certainly complex. The findings from two studies in particular (Gurin et al., 2011; Halualani, 2010) helped reveal the complexity inherent in the interactions between people from different cultural backgrounds.

Gurin et al. (2011) studied the effects of intergroup dialogue at the university level. They randomly assigned 52 groups of demographically mixed students $(n=1463)$ into either a dialogue course or wait-list control group. Each student completed surveys at the beginning and end of a term, and another one year later. Though the methods in 
the study were not reported in detail, Gurin et al. found that students enrolled in the dialogue courses were more cognitively open and viewed intergroup interaction more positively both at the end of the term and one year later. Gurin et al. concluded, "Participation in intergroup dialogue is an educational experience that builds social as well as personal responsibility for a more just society" (p. 51).

Halualani (2010) examined how multicultural university students make sense of intercultural interactions. Halualani interviewed 80 students over the course of two years about their experiences with intercultural interaction; 48 of the 80 interviews yielded significant information. Students define intercultural and cultural depending on which groups they see as different, how they value cultural groups, and how they place themselves within the "ethno-racial power hierarchy of the United States" (p. 255). The researchers identified three major themes: (a) participants frame culture around nationality or country of origin and positively view interactions with students from European and Asian countries; (b) participants view their interactions with students from the Middle East as uncomfortable and do not equate their cultures with country of origin; (c) some participants view Black/African American students as different and describe interactions with them as difficult. Together, the three themes show that students at a multicultural university view intercultural interactions differently depending on who is involved in the interactions and the socio-historical context in which the interactions occur.

Intercultural interaction is an umbrella term encompassing several different specific approaches to studying how people from different cultural backgrounds interact 
with each other. Two research traditions that have applicability to my topic are intergroup contact and intercultural communication.

Most clearly, intergroup contact appears to reduce prejudice (Allport, 1954), which has been the focus of most research in this area. The effects on reduced prejudice, though, appear to affect majority and minority population differently (Binder et al., 2009). In addition to reducing prejudice, intergroup contact may (a) lead to improved group work outcomes, (b) secure ethnic identities may be associated with increased acceptance of others, (c) students familiar with their ethnic identities may be more likely to be open to intergroup contact, and (d) intergroup contact seems to be associated with increased creativity and tolerance. In the following paragraphs, I describe the research behind these claims.

Intercultural communication is related to intergroup contact, but less focused on prejudice reduction and somewhat more action-oriented. Intercultural communication researchers focus on the features of linguistic and discursive contact between people from different cultural backgrounds (Gudykunst, 2005). Researchers typically examine intercultural communication through the historical and social contexts in which they occur, making them about more than just linguistic interaction (Piller, 2011). In addition, intercultural communication researchers often advocate for action or change in given situations.

Intergroup contact. The research on intergroup contact stems from the work of Allport (1954). Allport proposed that people are naturally prejudiced because we categorize our experiences; we view people we see as different less positively than our 
own groups. To lessen natural prejudice, Allport suggested four conditions that must be met in intergroup contact: (a) the people involved must have equal status, (b) they must have common goals, (c) there must be mutual cooperation, and (d) the contact must be supported by the authorities.

Many other researchers have examined Allport's (1954) claims to understand intergroup contact. In a meta-analysis of the literature on intergroup contact involving over 500 studies, Pettigrew and Tropp (2006) claimed wide support for the efficacy intergroup contact reducing prejudice, even without all of Allport's (1954) conditions being met. According to Pettigrew and Tropp (2006), the reduction of prejudice is greatest when all of the four conditions are met, but still works when one or more are absent. Pettigrew and Tropp reported on empirical studies written between 1940 and 2000 involving direct contact between distinct groups. They found that the greater the rigor in a study, the greater the effect of contact, with or without Allport's (1954) criteria. Importantly, the reduction of bias reaches beyond the immediate situation in which the contact occurred. Pettigrew and Tropp (2006) suggested more work be done to explore how the reduction of prejudice works in intergroup contact, as simple exposure may not be sufficient. Despite the wide range of studies included in their meta-analysis, Pettigrew and Tropp excluded all qualitative studies, perhaps reducing their ability to explore how intergroup contact works and almost certainly reducing their ability to explore the experiences of different participants.

Pettigrew and Tropp (2008) took up the challenge from their previous metaanalysis and explored mediators that lead to reduced prejudice on intergroup contact. 
They explored the efficacy of knowledge, anxiety reduction, and empathy and perspective taking. Of the three mediators, knowledge of other groups has the least efficacy; it does not significantly impact prejudice. Both anxiety reduction and empathy and perspective taking show much larger effects. Pettigrew and Tropp concluded that affective mediators are more effective than cognitive mediators in reducing bias and that many more mediators need to be explored. Like their earlier meta-analysis, though, all qualitative studies were excluded, limiting their ability to gain a full picture of how intergroup contact reduces prejudice, especially for distinct groups of participants. In their meta-analyses, Pettigrew and Tropp $(2006,2008)$ made clear the antiprejudice effects of intergroup contact, though they also emphasized that many mediators and other effects of intergroup contact deserve study. In a study that specifically explored the effects of indirect intergroup contact on prejudice among more than 1000 German adults toward Muslims, Pettigrew, Christ, Wagner, and Stellmacher (2007) found that both direct and indirect contact reduce prejudice, but only direct contact reduces individual threat from the outgroup. To increase acceptance of individuals, direct contact is necessary, though Pettigrew and Tropp (2008) called for further study in that area. Several studies have since explored various mediators, especially whether direct contact is necessary for prejudice reduction.

Ata, Bastian, and Lusher (2009) conducted a study that examined in-group attitudes toward Muslim minorities taking into account collective and individual threat. In a structured questionnaire using a sample of more than 900 secondary school children in Australia, Ata et al. found that having a Muslim friend had a drastic difference in 
reducing social distance toward Muslims. Ata et al. concluded that "promoting intergroup contact between Australia's school students will promote improved relations between Muslim and non-Muslim groups within the broader Australian community" (p. 505). This was one of the few studies of intergroup contact using participants who were not in college. Like the previous studies of intergroup contact, though, the data were entirely quantitative. Also, Ata et al. studied only the attitudes of the majority group, leaving one to wonder how the minority group experienced these interactions.

Binder et al. (2009) conducted a longitudinal study of more than 1000 secondary students in three European countries to examine if intergroup contact led to reduced prejudice or if low levels of prejudice led to increased intergroup contact. In their study, Binder et al. considered both majority and minority experiences with intergroup contact. They found that the quality of intergroup relationships had a greater effect on prejudice reduction than the quantity of intergroup relationships. Binder et al. also found that how minority members perceived their friends from majority cultures greatly affects their levels of prejudice. When minority students viewed their majority friends as typical of the majority, the effects of intergroup contact were greater. When minority students did not view their friends as typical, there was no effect to intergroup contact. As a result of these findings, Binder et al. concluded that, though intergroup contact can be beneficial for minority students, they react differently than what is reported in much of the literature that has focused on majority cultures.

In two concurrent studies, Bastian, Lusher, and Ata (2012) focused on the effects reduced social distance, which they described as the real world application of attitudes, 
might have on the effects of intergroup contact. Bastian et al. first studied 750 secondary students from a variety of backgrounds using a questionnaire to measure their social distance to Muslim Australian students. They found that having a Muslim friend was significantly associated with positive views of Muslims in general. In their second study, Bastian et al. used a questionnaire with 277 Muslim secondary students from Australia to examine minority students' social distance to majority Australians. They found that while intergroup contact did not affect how minority groups viewed the majority, it did increase the desire for minority group members to engage in further contact with members of the majority group. In short, though intergroup contact did not reduce negative perceptions from minorities toward the majority, it led to the desire for more contact.

Most studies exploring intergroup contact have examined the effect of contact on prejudice. A few studies have examined other effects of intergroup contact. In an instrumental case study, Cotton, George, and Joyner (2013) examined group work among students at a multicultural university in the United Kingdom. They assigned students to three different groups; two of the groups had mixed majority and minority students. The other group was made up of all international, non-native-English-speaking students. Cotton et al. found that students from the United Kingdom spoke more than minority students. Also, students from the United Kingdom had less favorable views of group work than minority students. Cotton et al. recommended that for group work to be maximally beneficial for minority students, teachers must focus on classroom 
management during group work, groups should have specific roles, and teachers should minimize assessment of the outcomes in favor of the process.

Phinney et al. (2007) examined the role of ethnic identity in evaluations of intergroup contact in two studies. In the first, they surveyed over 700 college freshmen at a large, urban, commuter university and found that minority students with more secure ethnic identities were "more open and accepting of members of other ethnic groups" (p. 483) than students who were not secure in their own ethnic identities. Phinney et al. followed their first study by interviewing 119 adolescents from five ethnic groups in diverse high schools in and around Los Angeles to gain a better understanding of how ethnic identities relate to intergroup attitudes. They found that adolescents with secure ethnic identities were more likely than students without secure ethnic identities to think about intercultural interactions and to situate their intercultural experiences into a wider context; they understood how intercultural interactions shaped their senses of identity.

Leung and Chiu (2010) reported on five studies examining multicultural experiences on creative performance and psychological processes underlying creativity, as well as limiting factors to creative benefits of multicultural experiences. They used quantitative coding in each study and generally found that multicultural experiences increased creativity. The first study, in which 65 European-American undergraduates watched either one or two presentations about cultures, then were asked to complete a creativity task, showed exposure to different cultures activates creativity. The second study, in which 72 European American undergraduates listed gifts they would give to others, showed that people who have had multicultural experiences were more creative in 
gift-giving than those without multicultural experiences. The third study, in which 83 European American undergraduates were asked to creatively design a research project regarding happiness after reading the backgrounds of multicultural authors, showed that the more multicultural exposure a person has, the more creative they are. The fourth study, in which 64 European American undergraduates were given the same task as in the third study and split into high-pressure and regular groups, showed that people with more multicultural experience are more likely to be creative in unfamiliar situations, except when they are under time pressure. The final study, in which 64 European American undergraduates were given the same task as the previous two studies and half were asked what would happen to their bodies around death, showed that people with multicultural experiences view American sayings more positively than those without multicultural experiences. Overall, Leung and Chieu's five studies indicate that intergroup contact is associated with increased creativity and openness, but none of the studies examined qualitative aspects of these traits.

Tadmor, Hong, Chao, Wiruvhnipawan, and Wang (2012) specifically examined the effects of multicultural experiences on intergroup bias using a cognitive componentneed for cognitive closure. Reporting on six studies involving between 69 and 89 participants in Israel and the United States, the authors demonstrated that multicultural experiences have universal benefits, even without direct contact. In addition, they found that intergroup contact could reduce bias that has a cognitive component. Generally, multicultural experiences, "cultivate a generalized tendency for a more tolerant mind" (p. 767). Like the previous studies, Tadmor et al. (2012), did not examine the experiences of 
the participants in the studies. As stated earlier, different participants have different experiences, so it is important to qualitatively examine how their communication works in different situations.

In sum, intergroup contact clearly reduces prejudice (Allport, 1954), but there are many mediators making the exact processes very complex (Pettygrew and Tropp, 2008). Many of the studies were conducted at the university level, limiting their applicability to a middle school context. In addition, the actual experiences of the participants in these studies were rarely examined; a qualitative approach exploring how participants feel about the intercultural interactions in which they engage may help shed more light on what happens in these interactions. Intercultural communication, because of its increased focus on linguistic contact, lends itself to qualitative approaches examining how participants experience their interactions.

Intercultural communication. Intercultural communication is a field related to intergroup contact, but focused on linguistic contact between groups. Because I conducted a study involving intergroup contact between participants who spoke different languages in their homes, it is important that I took into account their linguistic differences, even though they spoke mostly English in their classes. There are many different theories of intercultural communication (Gudykunst, 2005) giving different lenses through which to view participants' experiences. However, because I am not focusing on just the linguistic connections between students, I am treating the field as one body. It is important to note that intercultural communication does not need to focus 
solely on linguistic contact at the expense of cultural and historical factors. Piller (2011) very clearly critiqued the overemphasis on language:

My disappointment with much of the intercultural communication literature stems from the fact that people with diverse backgrounds leading linguistically and culturally diverse lives and engaging in linguistically and culturally diverse relationships...hardly ever seem to figure in the intercultural communication literature. (p. 1)

According to Piller, intercultural communication is, "communication where language learning and language proficiency on the one hand and stereotyping and the discursive construction of identity on the other intersect with social inclusion and justice" (p. 3). In addition, "there is the obvious point that cultures meet and mingle in a specific historical, social and economic context" (p. 27). In short, intercultural communication is interaction between people from different cultures who speak different languages embedded in social and historical contexts. Many intergroup contact studies lack a call to action that is prevalent in intercultural communication research. Studies on intercultural communication tend to focus on helping people improve their situations through effective communication, whereas studies on intergroup contact tend to only describe the effects of interaction. A portion of many calls to action is to point out differential power relationships between participants in intercultural communication.

For refugee students, most intercultural interactions are with people who have more cultural capital than they do. For refugee students to gain cultural capital in a multicultural setting, they must be able to effectively communicate with individuals from 
other cultural backgrounds. When language differences come into play, effective communication may be difficult. Verschueren (2008) examined three ways meaning could be generated in intercultural communication under conditions of asymmetrical power relationships. He framed the argument into, "three fields of tension that emerge in the way in which meanings are generated" (p. 27). The three fields of tension are: "the tension between communicative intentions and inferencing processes; the tension between culture-related assumptions and what is actually said; and the tension between legal frames of interpretation and the intrinsic properties of life stories" (p. 21). Overall, Verschueren echoed multicultural education in recommending educators recognize and incorporate into their lessons the ethnic and cultural differences among their students. Zimmerman (1995) conducted a study in which 101 international university students from 35 different countries at a university in the Midwest were interviewed. The students, who ranged in age from 17 to 41 years old, had been in the United States from three months to eight years. The interviews were conducted by trained undergraduate students and were structured as Likert-type questionnaires rather than open-ended interviews. Zimmerman found that international students' perceptions of their own English proficiency was somewhat related to their adjustment to life in the United States. More significantly, according to Zimmerman, "The most important factor in international students' adjustment to American culture was frequency of interaction with American students" (p. 329). International students' level of comfort was unrelated to the amount of time they have lived in the United States, but highly related to the amount of time they spent interacting with American students; the more intercultural interaction, the more 
comfortable international university students felt. Zimmerman recommended that universities set up programs that encourage international students to interact with American students. Though not specifically examining early adolescents, the same principal could be applied in middle school, when peer interaction is already an important part of students' development.

Though Zimmerman's (1995) study indicated increased interaction and communication help international students feel more comfortable in the United States, all of the students involved were voluntary immigrants, who have different experiences than involuntary or refugee immigrants (Ogbu \& Simons, 1998). Cheah, KaramehicMuratovic, Matsuo, and Poljarevic (2011) examined the role of intercultural communication in the resettlement process of Bosnian refugees in St. Louis. They gave a questionnaire to 315 Bosnian refugees who had resettled in St. Louis between 1991 and 2006. Nearly all (97\%) of the participants identified as Muslim and had an average age of nearly 40. Cheah et al. found that as the participants' competence in English increases, so does their interpersonal relationships, familiarity with cultural practices, and connection with cultural norms in the United States. Participants' interpersonal relationships are significantly correlated with their connection with U.S. cultural norms, but not familiarity with cultural practices. In addition, as the participants increased their relationships with fellow Bosnians, their connection to their own cultural background increased. Last, as participants grew more confident in English and formed more connections with both Americans and Bosnians, their psychological health improved and they held a stronger connection to their Bosnian heritage. In summary, learning the 
language of, building connections with people from, and learning the norms of the host country, while also maintaining connections with their ethnic background helped Bosnian refugees adjust to life in St. Louis. However, the participants were far from adolescent ages.

As helpful as it might be for immigrants to engage in intercultural communication only with people from the host country, in my school context students have many different opportunities for intercultural communication with people from various cultural backgrounds. Uryu, Steffensen, and Kramsch (2014) studied a conversation between four highly educated adults (all had earned at least an M.A.) from three different countries (Germany, Russia, and two from Japan) who all spoke fluent English, though it was not their first language. Uryu et al. used stimulated recall, journals, and ethnographic interviews to deeply examine a conversation about Thanksgiving between the four adults. The conversation and subsequent analysis revealed that intercultural communication is decidedly more complex than the non-native-English speaker learning the norms of the host country. Meaning was continually co-constructed as each speaker worked to make sense of the others' intended meanings based on their sociohistorical backgrounds. Even though Uryu et al. studied educated adults, not middle school students, their conclusions point out that the meanings that emerge from intercultural interactions are not necessarily predictable and may be interpreted differently by the different participants involved based on their backgrounds and experiences.

Summary. In a multicultural setting, students have opportunities to interact with students from various ethnic backgrounds. These interactions, studied under the terms 
intergroup contact and intercultural communication, can reduce prejudice (Allport, 1954), lead to better work outcomes (Cotton et al., 2013), help form secure ethnic identities

which leads to more openness to further intergroup contacts (Phinney et al., 2007), and increase creativity among those involved (Leung \& Chiu, 2010). Ultimately, these interactions may lead to increased cultural capital and greater access to the benefits of the hegemonic culture.

Specifically for refugees, maintaining connections to their home culture while also learning the language and norms of the host country aids in adjustment (Cheah, Karamehic-Muratovic, Matsuo, \& Poljarevic 2011). In the next section, I address the experiences of some of the most marginalized students in schools in the United Statesrefugees.

\section{Refugee Students}

Refugee students enter school in the United States having experienced significant trauma, coming from vastly different cultural systems, lacking the ability to speak fluent English, and often living in poverty (McBrien, 2005; Valenta, 2010). To achieve success with such overwhelming challenges, refugee students must learn the norms of the hegemonic culture. To be successful in school, refugee students must adopt the accepted habitus in the fields of their classrooms.

In a widely-cited study examining how second generation immigrant children adapt to their new countries, Portes and Zhou (1993) provided examples of Mexican immigrants in a small coastal California town, Sikh immigrants from Punjab in a rural California farming community, and Caribbean immigrants in Miami. They used these 
examples to present their theory of three different ways second-generation immigrants acculturate to their new country. One way was to rapidly assimilate into the mainstream middle-class. That, however, was not always an option. A second way was for immigrant children to reject the mainstream and remain closely tied to their own culture. This option could result in perpetual discrimination and subordination. A third way immigrants acculturated was to utilize the resources of their own community to succeed and slowly acculturate to the larger society. Essentially, immigrant students become successful in their new country in a variety of ways, which largely depend on their unique situation. Isolating oneself from the larger community, however, is destructive. Though Portes and Zhou have been widely cited in research on refugees, they did not distinguish between immigrants and refugees, a difference could affect how people tend to adapt to their resettled homes.

Though researchers often refer to immigrants and refugees together, there are differences between the groups. Taylor and Sidhu (2012) argued that policymakers and researchers have ignored the needs of refugee students because they have been grouped with voluntary immigrants. Cheah et al. (2011) clearly stated that refugees should be viewed differently than immigrants; "As refugees leave their countries of origin due to fear of persecution, their experiences are generally different from immigrants" (p. 220). Valenta (2010) distinguished refugees from immigrants in how they identify as being marginalized in the larger society. Immigrants typically anticipate a long stay in their new country, whereas refugees may not. Because some refugees want to return to their countries of origin, they do not react to marginalization by wanting to integrate into the 
mainstream society. Ogbu and Simons (1998) made clear that the educational implications for refugee students are very different than for voluntary or involuntary immigrants.

Ogbu and Simons (1998) classified three types of immigrants: voluntary, involuntary, and a group in between. Refugees fall in between. Voluntary immigrants choose to enter their new countries and typically do well in school over time. Involuntary immigrants were taken over by a country and do not typically improve their school performance over time. Refugees have traits of each of the other groups. According to Ogbu and Simons (1998), voluntary immigrants may experience initial difficulties in school, but over time their school performance improves to the point of being indistinguishable from the majority population. Involuntary immigrants, because they were forced to become part of the United States against their will and have "more persistent cultural and language difficulties" (p. 166), typically fare worse in school and do less well economically than other immigrants. Refugees typically enter the United States with interest in learning the cultural norms and language while also maintaining their own culture. Ogbu and Simons stated that refugees and migrant workers, "tend to learn only as much of their host's culture and language as necessary to achieve their temporary goals, which may not include school credentials" (p. 165). Because their situation is inherently temporary, they may do well in school, but also may not invest enough to achieve success.

Despite some conflating of immigrants and refugees in the literature, and various suggestions of what the differences may be, there is a clear definition of what constitutes 
a refugee. According to the UNCHR (2005), based on Article I of the Convention Relating to the Status of Refugees, a refugee is anyone who cannot return to his or her country, "owing to well-founded fear of being persecuted for reasons of race, religion, nationality, membership of a particular social group or political opinion..." (UNHCR, 2005, p. 5). Furthermore, the 1969 Organization of African Unity (OAU) Convention added that refugee status should also apply to those who, due to external forces (e.g., foreign invasion, occupation) are forced to leave their country of origin. Article III of the 1984 Cartagena Declaration on Refugees broadened the definition of refugee to those who fear for their lives for human rights violations, general violence within the country, or threat of foreign invasion (UNHCR, 2005). In short, a refugee is someone who was forced to leave their country of origin due to fear for their lives or fear of persecution. McBrien (2005) summed up many of the major barriers refugee students face upon relocation and how difficult their lives can be. She stressed that the accumulated traumas prior to achieving refugee status by families had a large effect on students' ability to be successful. Families were often, then, placed in high-poverty urban areas, which led to children rejecting their family to fit in with their peers. Students who performed best in schools were the ones who best learned the language and culture of the dominant society.

Based on her literature review of the refugees in the United States, McBrien (2005) made several recommendations. First, students must learn the new culture and language while retaining their original language and culture. Second, students need social support to navigate potentially conflicting messages from their family and schools. 
One of those supports is to connect parents to schools. Another is to support students in school through instances of structural and personal prejudice. A final support is teachers confronting their own attitudes and moving toward multicultural education for every student. Key to this support is helping teachers understand the situations of refugee students. As areas for further research, McBrien (2005) suggested researching specific ways to boost the academic achievement of refugee students and examining more closely the experiences of specific groups of refugees, because wide variances exist among different refugee populations.

In a more recent study, Dryden-Peterson (2015) examined quantitative UNHCR data regarding the educational experiences of refugee students. She found refugee students enter their countries of resettlement having experienced limited and interrupted schooling, language barriers to educational access, poor quality instruction, discrimination. Dryden-Peterson recommended educators (a) be aware that refugee students may have never experienced a child-centered classroom, (b) talk with refugee students and their families about any wariness they have or discriminations they may experience, and (c) be cognizant of refugee students' backgrounds in making placement decisions.

Researchers have examined several different supports such as the ones McBrien (2005) and Dryden-Peterson (2015) suggested for refugee students. Many programs of various levels are available to students from refugee families. Some programs connect families to the wider community; others are school-wide; still others are implemented in 
classrooms. Because each of my participants was female, after I report what is known about supports for refugee students, I detail the research about female refugee students.

Community-based and school-wide programs. Multicultural education researchers have advocated for holistic changes to education to support a multicultural population (Banks, 1993; Gay, 2004). Holistic supports for refugee students can include community-based programs and school-wide programs. I am grouping them here because they are often connected, leading to a wrap-around effect in which communitybased programs have influence in the school and school-wide programs reach out to the community.

McBrien and Ford (2012) examined the efficacy of a program for refugee families in the United States. They were especially interested in the school liaison program, which attempts to bridge families, school, and other community services. Using a mixedmethods approach, including surveys, focus groups, and open-ended survey questions, McBrien and Ford concluded that the liaison program resulted in, "significant positive changes in attitudes, beliefs, and behaviors" (p. 122). They recommended expanding parent, school, and community connections to support refugee children in schools, whether or not a program is available that does so.

Though McBrien and Ford (2012) advocated creating community connections to help refugee students, most studies centering on how to support refugee students have focused within the school, though with some mention of community connections. Pugh, Every, and Hattam (2012) studied a South Australian primary school that claimed to have a whole school approach to educating their refugee students. The whole school approach 
included the structure, culture, and pedagogy of the school. They focused on the structure of the school in their paper. They interviewed the principal, assistant principal, and teachers, observed classes, staff meetings, the staff room, and an inquiry group, and gathered various documents to assess how the structure of the school addressed the needs of refugee students. Pugh et al. found several positive structural features for refugee students including organization that promotes intercultural interaction, distributed leadership, community connection, effective professional development for teachers, language and counseling support, and localized adjustments to standardized curriculum. They recommended increasing funding for support, placing teachers based on skill set, decoupling school funding from class sizes, and increasing the localization of curriculum.

Like Pugh et al. (2012), Taylor and Sidhu (2012) focused on a school-wide approach in a case study examining how four purposefully selected schools (one public and three Catholic) in Australia best supported refugee students. They interviewed staff members and analyzed documents to identify features that support refugee students. Taylor and Sidhu found seven school policies that lead to success for refugee students: targeted policies with support, commitment to social justice throughout the school, a holistic approach to education, strong leadership, a school-wide inclusive approach, support for specific language and social needs of refugee students, and connections with outside agencies. Especially important, according to Taylor and Sidhu, is that schools adopt an inclusive, school-wide approach that encourages students to interact and work together. Though this study was small and used a sample that is not necessarily 
representative of education as a whole, it provides models for how refugee students can be supported in schools, including encouraging school wide intercultural interaction.

Nwosu and Barnes (2014) also examined how a particular school environment could support refugee students. They studied how a charter school for refugee students in Canada provided support in students' ethnic identity development through a multicultural approach. The school context was unique in that it was an elementary designed for refugee students from two different communities and staffed by a diverse cross-section of adults. Nwosu and Barnes conducted interviews with five purposively selected staff, reviewed documents, and observed the participants. They reported that the cultural diversity of the students were foregrounded via a multicultural approach to education, leading to strong ethnic identities and desires to work for social change among its students. Nwosu and Barnes contended that the participants developed agency resulting from an inclusionary approach that valued each student's background and made each student feel part of the school community.

Block, Cross, Riggs, and Gibbs (2014) took a wider approach than either Taylor and Sidhu (2012) or Nwosu and Barnes (2014) in examining how schools in Australia could be structured to support refugee students. They conducted an independent evaluation of the School Support Programme, which had the goal "to establish a more coordinated system of inter-sectoral support between community, education and government sectors to address the well-being and educational needs of refugee children and young people" (p. 5). Block et al. examined 38 schools (of 44 that were initially involved) by surveying and interviewing people who were partnered with the school to 
provide support to refugee students. They found that a holistic model supporting learning, social and emotional needs, inclusiveness, and cultural diversity was best. To implement the program most effectively, they recommended streamlining and differentiating training, deeply involving educators throughout the process, various layers of oversight to provide coherence throughout the school, and increasing funding to expand the program.

While McBrien and Ford (2012), Pugh et al. (2012), Taylor and Sidhu (2012), Nwosu and Barnes (2014), and Block et al. (2014) consistently supported a holistic approach to educating refugee students, they did not offer many suggestions of what might work in particular classrooms. Unfortunately, because the needs of refugees rarely are strong in the public consciousness, little is known about what might work in particular classrooms.

Pinson and Arnot (2007) found that discussions of issues close to those of refugee students in the classroom are rarely integrated into the general public, and therefore, receive little attention. Though they did not focus on school-aged populations, they echoed the previously-cited researchers in advocating a child-centered holistic approach to the education of refugee students. Pinson and Arnot, though, questioned whether there is a need for more research regarding how school districts are implementing education for refugees and what programs are most effective. Among the suggestions by the researches who examined community-based and school-wide programs were several that could be implemented in the classroom. Certainly it is worth reviewing what researchers currently say about what classroom-level child-oriented approaches benefit refugee students. 
BURMESE REFUGEE STUDENT INTERACTION

Classroom-based practices. One theme common to both multicultural education and research on refugee students is that schools need to help marginalized students feel valued for what they bring to the classroom. Helping refugee students feel valued in the classroom is a strong focus, along with increasing intercultural interaction, of those who have researched classroom-based supports for refugee students.

Dooley (2012) examined a very specific approach to help students feel more valued in their classrooms. She interviewed teachers, administrators, and paraprofessionals regarding their approaches to teaching eight Eritrean, Sudanese, Rwandan, and Burundian middle school students in an Australian school. Eighty percent of the students were refugees, mostly from Africa. Seventy percent of the refugee students had little, no, or severely interrupted schooling. Dooley reported, "Teachers were enabling refugee students from Africa to participate in the intellectual work of the classroom” (p. 10) by connecting students' experiences to conceptual knowledge and helping students understand different processes of knowledge that are valued in the classroom. Though Dooley indicated success in increasing the value students felt in the classroom, all of the interviews were conducted with adults and it is never directly stated that students felt more valued.

While Dooley (2012) only interviewed adults, Dávila (2012) used ethnographic observation and interviews to study eight Vietnamese refugees resettled in the United States about the challenges they faced in a large, high-poverty, urban high school in the southeast. Viewed through the lens of Bourdieu's (1977) theory of field, Dávila (2012) found that being educated meant being able to navigate the system effectively, which left 
refugee students "uncertain about their ability to succeed in school or beyond" (p. 144). In addition, refugee students thought learning English equated with academic success. However, because they were typically well-behaved, they did not have ample opportunities to engage with the teacher or other students, which limited their Englishlanguage acquisition. Dávila also found that students were anxious about one-size-fits-all expectations and requirements they were expected to complete. Refugee students were marginalized in their mainstream classes, but there were other places in school (e.g., ESL classes, Junior Reserves Officer Training Corps, a soccer team) in which they felt valued. Like Dooley (2012), Dávila emphasized helping students feel valued in the classroom. She recommended several curricular changes to better connect refugee students to school so that they feel valued and are able to succeed. Among Dávila's recommendations was increased intercultural interaction, which has also been promoted by other researchers.

Kirova (2012) also advocated intercultural interaction in educating refugee students and helping them feel valued in the classroom. She used a variety of qualitative methods such as focus groups, individual interviews, observation, field notes, and document analysis to study the efficacy of a bilingual program involving four different languages (i.e., Somali, Arabic, Kurdish, English) in a kindergarten to grade 9 Canadian public school situated in a low income, ethnically, linguistically, and racially diverse area. From 2007 to 2010,52 students received bilingual instruction in the presence of the students from other cultural backgrounds. Kirova stated, "The fusion of cultures that occurred in this shared space was seen as an opportunity for multiple, even contradictory, cultural identities to coexist and develop" (p. 39). Though the school served students 
from kindergarten through ninth grade, Kirova emphasized early childhood progress, limiting its applicability to students in middle school.

In a study of 83 adolescent refugees from the former Republic of Yugoslavia resettled in Australia, Kovacev and Shute (2004) examined how interactions with peers from outside their ethnic background impacted their adjustment to their host country. The authors quantitatively measured adjustment through questionnaires about global selfworth and peer social acceptance. They found that adolescents with strong relationships with peers from their own ethnic background and those from the host country adjusted better to host country.

Though not directly advocating intercultural interaction, Banki (2012) indicated that intercultural interaction was a key part of helping refugee students feel valued and improving education for all students. Banki focused on the positive skills refugees from Burma and Bhutan bring to the classroom and how their interactions with the rest of their classes affect the educational experiences for all. She identified three general areas: contextual, practical, and social. The interactions among students including those from Burma and Bhutan can help contextualize world events for all students. Because refugees typically immediately apply the trainings they receive (such as how to fill out resettlement paperwork), they can help all students better understand to employ newly acquired skills. Refugees from Burma and Bhutan can also help all students understand how to navigate difficult social relationships because of their experiences in refugee camps. Banki concluded that the benefits she shared are not comprehensive and need 
further study to be most effectively implemented, but could effect change for both refugees and non-refugees alike.

Like Banki (2012), Ferfolja and Vickers (2010) suggested that improving the educational environment for refugee students could have benefits for others. Ferfolja and Vickers found benefits for preservice teachers. They examined the efficacy of an Australian program in which preservice teachers tutor refugee students in grades seven through 11. They interviewed the coordinating teachers about their perceptions of the success of the program. In addition, they conducted before- and after-questionnaires exploring literacy levels of the refugee students involved. Ferfolja and Vickers concluded that one-on-one tutoring led to success for refugee students and transformed the perceptions of teaching for the preservice teachers. In theory, those pre-service teachers might be able to more effectively reach other marginalized students in the future, improving education for all.

Clearly, refugee students can be successful if supported by feeling valued in the class (Dooley, 2012) and engaging in intercultural interactions (Banki, 2012; Dávila, 2012; Kirova, 2012; Kovacev \& Shute, 2004). Both feeling valued and engaging in intercultural interactions help them navigate unfamiliar norms and, presumably, gain cultural capital. Interestingly, Ferfolja and Vickers (2010) suggested that improving education for refugees may have benefits for non-refugees as well.

Most studies about refugee students do not report the genders of the participants. In my study, each of the participants was female. Next, I discuss the research about female refugee students. 
Female refugee students. Because my study focused on three Burmese refugee females, I searched for information regarding adolescent female refugee students. I was able to find very few studies specifically addressing the experiences of female refugee students. The participants in most of these studies were refugee students from Africa. In one study, the researcher interviewed Bosnian refugees.

In a study of seven female Somali refugee students in an urban high school in the United States, Oikonomidoy (2007) used observation and focus groups to find the participants banded together and helped each other to respond to religious discrimination and learn English to navigate the school system. They proudly displayed their identities as Muslim by wearing headscarves, but they did not wear other traditional clothing, indicating a balance between carving out ethnic identities separate from the mainstream culture in the United States and fitting in with their mainstream peers. Oikonomidoy also found the students helped each other learn English to navigate school collectively in the United States.

In another report on the same study, Oikonimodoy (2009) reported female Somali refugee high school students partially assimilated to the school culture to fit in, but maintained identities outside of school tied to their cultures of origin. They reported wanting to use the education they receive in the United States to help their ethnic community and ultimately return to Somalia.

In her dissertation study, Harris (2010) found that female Sudanese refugee students in high school actively oppose their marginalization by redefining themselves in positive terms. They consistently felt misrepresented with negative images, which led to 
poor treatment at school. To counteract their misrepresentation, the girls focused more on their future than their past. Harris advocated providing space for these students to share their stories.

In the same study reported in the identity formation section Mosselson (2006) interviewed 15 Bosnian refugees between the ages of 15 and 24 who lived in New York. In addition to developing the roots \& routes framework for refugee identity formation, Mosselson proposed a model of why female refugee students might do well in school she called "Masks of Achievement" (p. 25). In this model, female refugee students are academically successful partially, "to hide depression, post-traumatic stress disorder (PTSD), loneliness, and so on" (p. 25). In addition, academic success serves as a way for these students to connect with other students and teachers. In the study, academic success was crucial in the participants' identity formation and adjustment to life in the United States.

In sum, female refugee students, similar to the description of refugees by Ogbu and Simons (1998), held on to pieces of their places of origin while also looking toward the future and working to become successful in their new countries. Overall, refugee students want to be academically successful (Mosselson, 2006). In working to do so, they benefit from supports provided by schools, such as teachers helping them feel valued (Dooley, 2012), and encouraging intercultural interaction (Banki, 2012; Dávila, 2012; Kirova, 2012; Kovacev \& Shute, 2004).

One flaw in some of the research on refugees is that they are grouped together without regard for the individual needs of specific populations (McBrien, 2005). It is 
important that researchers explore the differing needs of refugee populations so that educators can tailor their support most effectively. Burmese refugees, because of their recently high numbers of entry into the United States (Martin \& Yankay, 2012; UNHCR, 2015), are one of the groups that has been especially important to study in recent years. Though they are a diverse group consisting of many different ethnicities, they are classified by the UNHCR as a single refugee group. In an effort to understand the experiences of this refugee groups, I describe the research on Burmese refugees.

\section{Burmese Refugees}

Though Burmese refugees constitute a fairly small number of the students at the school the participants attended, they are one of the largest incoming refugee groups in the United States in recent years (Martin \& Yankay, 2012; UNHCR, 2015). A relatively small amount of research has been conducted on Burmese refugees, especially those in middle school. The scant research gives some insight into the backgrounds and experiences of this group of refugees.

As stated earlier, the Burmese are people from Burma, also known as Myanmar, which had been under oppressive military rule for about 50 years (Ranard \& Barron, 2007, p. 1) until a move toward democracy beginning in 2011 (Nilsen, 2013). Of the many ethnic groups classified by the UNHCR as Burmese (2005), the largest is the Burmans (Ranard \& Barron, 2007), but others include Karen, Karenni, Rohingya, Shan, and Chin (Fuertes, 2010; Ranard \& Barron, 2007).

Much of the literature concerns people from the Karen ethnic group and how they experience various facets of being away from their home countries. Karen is often used 
as an umbrella term for ethnic groups speaking similar language, including Skaw, Pwo, Kayah (Karenni or Red Karen), and White Karen (Oh, 2012; Ranard \& Barron, 2007). They were described as an ethic group as far back as the mid-19 $9^{\text {th }}$ century (Cross, 1854). Even then, Cross recognized that the term Karen referred to, "perhaps three or four distinct tribes" (p. 291). In the literature about the Karen, themes of resiliency and strength are common.

Fuertes (2010) interviewed and conducted workshops with 15 Karen refugees in a camp in Thailand. The participants described themselves as "birds inside a cage" (p. 20), in that they were trapped in the camp and unable to fly, or be self-reliant as they had once been. Due to their lack of opportunities in camp, some of the people wanted to seek asylum elsewhere.

Oh and van der Stowe (2008) also examined the experiences of 182 Karen refugees in six predominantly Karen camps on the Thai-Burmese border. They followed up by interviewing 28 pregnant and/or married women across the six camps about their educational experiences. Oh and van der Stowe found that education in the camps for Karen children may have been better than in Burma; however, it was not as robust as for non-refugee students outside the camps in Thailand. In addition, teaching methods and language use in the camps were not inclusive and access to schooling for students with special needs was challenging. Because the educational opportunities in camps, though better for Karen refugees than those in Burma, were severely lacking in Thailand, one wonders how Karen refugees fare after being granted asylum elsewhere. 
In two studies, researchers examined how Karen refugees have dealt with resettlement. Bird, Brough, and Cox (2012) argued that Karen refugees in Brisbane, Australia have united and formed their identities because of centuries of socio-historical events, including persecution, proselytization, colonization, and nationalization. As a result, the Karen in Brisbane have successfully resettled through forming self-reliant groups. Gilhooly and Lee (2014) examined how three Karen refugee brothers used technology to find their places in their resettled homes and maintain connections with their culture. They used internet access to maintain connections with friends, relatives, and strangers who had resettled elsewhere. Through these connections, they built ethnic solidarity, especially around political events. They were also able to creatively express themselves through online media. Though they did not have direct access to a Karen community, they were able to access it online, which, according to Gilhooly and Lee, aided in their adjustment to their new home.

Another ethnic group that has received attention from researchers is the Rohingya. The Rohingya are from Rakhine, or Arakan, state in the western part of Burma. Their refugee camps are typically in Bangladesh (Kiragu, Li Rosi, \& Morris, 2011; Ragland, 1994: Rahman, 2010). Rahman (2010) referred to the Rohingya as "among the most unfortunate ethnic minority groups in the world" (p. 233). Letchamanan (2013) called them a people without a state due to their lack of official recognition in Burma. Because the Rohingya are Muslim and many speak Bengali, they are an ethnic, linguistic, and religious minority in Burma and Bangladesh and have been badly mistreated in both countries (Kiragu et al., 2011; Rahman, 2010). 
Other groups from Burma include the Karenni, Chin, and Shan. Ranard and Barron (2007) described these three groups in detail. The Karenni come from the smallest state in Burma and are a seen as similar to the Karen. They are traditionally animist, but many are Christian today. As refugees, the Karenni have similar experiences as the Karen, but with fewer supports. For example, in my school, the Karenni refugees have had no one to translate for them at conferences except family members. The Chin constitute a variety of different related ethnic groups with different languages. They come from a region bordering Rakhine state and Bangladesh, where the Rohingya originate. Most Chin today are Christian. According to Ranard and Barron, most Chin who fled Burma reside in Malaysia, where they receive no specific support or protection. The Shan come from northeastern Burma and are traditionally Buddhist. Many Shan have been forced to flee to Thailand, but are not recognized as refugees by the Thai government.

Though refugees in camps come from very different ethnic backgrounds, they often have similar perceptions of life in the camps. In a series of interviews with Karen and Chin refugees in Australia, Rosbrook and Schweitzer (2010) found that both conceptualized home similarly. Home was perceived as the psychological space of safety and retreat, socio-emotional relatedness to family, and as a geographical-emotional landscape.

Similar to the literature about the Karen focusing on their experiences away from their home countries, much of the sparse literature regarding Burmese refugee students concerns their experiences in their countries of resettlement. According to Valenta 
(2010), however, refugees' experiences in the countries of resettlement are strongly affected by their biographies, including their experiences in refugee camps. Oh (2012) examined the experiences of Burmese refugee students in seven predominantly Karen refugee camps in Thailand. Because the camps were supposed to be temporary and travel was restricted, children from them were not allowed in Thai schools. People in the camps reacted by forming their own schools, which came to be supported by NonGovernment Agencies (NGOs) and minimally affected by the Thai government. Within the seven camps Oh examined, there were 70 schools with over 1,600 teachers and 34,000 students. The dominant structure of the schools was that of Christian Karen, and the dominant language is Skaw Karen. According to Oh, "Education management is dominated by Christian Skaw Karen-speaking elites" (p. 75). Though most people in the camps were Karen, there were many students who are not Christian Skaw Karenspeaking who were disadvantaged by their dominance. Included in the disadvantaged group were Muslim children who attend Burmese language schools. No secondary schools for children who attend Burmese language schools existed, meaning that far more Christians attended secondary school than non-Christians. Among most residents of the camps, education was highly valued, but many groups were disadvantaged by the educational domination of Christian Skaw Karen-speaking residents. Students in my classroom may have been advantaged or disadvantaged in their refugee camps, which is important for their navigating their new school experiences.

Letchamanan (2013) examined the educational experiences of Rohingya students in refugee camps in Malaysia. She concluded that schools for Rohingya students were 
inadequate. The facilities were crowded and noisy due to multiple classrooms within one classroom area. Materials were not tailored to the students, and teachers were illequipped to meet the needs of disadvantaged students.

Purkey (2006) studied the educational experiences of Burmese refugees in Thailand, but did not specify the particular ethnic groups in the camps. Like Oh (2012), Oh and van der Stowe (2008), and Letchamanan (2013), Purkey (2006) found the schools in refugee camps severely lacking in materials, training, and support. She recommended that these deficiencies be address to improve the lives of Burmese refugees.

The aforementioned studies focused on educational opportunities in refugee camps. Very few researchers have examined the experiences of Burmese refugee students once they are granted asylum. One such study was conducted by Isik-Ercan (2012), who interviewed 25 Burmese refugee families about the experiences their students had in Midwestern elementary schools. Overall, the families trusted the schools, but did not feel connected to them. One reason for the lack of connection was differences between the norms of the Burmese families and U.S. schools. Isik-Ercan made several recommendations, including increased advocacy for bilingualism and parent participation, community-based education and academic support, and increased cultural responsiveness within schools. These recommendations echo those from other refugee and multicultural education researchers.

Though Burmese refugees encompass several different ethnic groups, they are classified together according to the UNHCR (2005), and have some similar traits. They tend to come from camps lacking sufficient educational opportunities (Letchamanan, 
2013; Oh, 2012; Oh \& van der Stowe, 2008; Purkey, 2006). Once they leave their refugee camps, they may struggle and feel disconnected from schools (Isik-Ercan, 2012), but they can be successful by creating solidarity amongst their own groups (Bird et al., 2012; Gilhooly \& Lee, 2014). Though not yet studied for Burmese refugees, one way to help ease the disconnect between home and school may be to increase opportunities for intercultural interactions in school.

\section{Summary of Literature Review}

I used Bourdieu's (1973, 1977, 1990; Bourdieu \& Wacquant, 1992) theory of field and theory of social and cultural reproduction to examine the experiences of Burmese refugee students in a multicultural middle school classroom. In short, Bourdieu stated that in certain fields, such as a classroom, specific dispositions are more successful than others. These dispositions get codified as habitus. The more closely one's behaviors match the accepted habitus, the more successful a person becomes. People gain capital when their behaviors closely match the accepted habitus.

To learn successful habitus in multicultural middle school classrooms and gain cultural capital, Burmese refugee students could benefit from intercultural interactions. Research indicates refugees enter the United States with a variety of challenges when it comes to accessing the cultural capital necessary for success (Dávila, 2012; McBrien, 2005; Taylor \& Sidhu, 2012).

Adolescence, especially during middle school, is a time during which children explore their places in the world, particularly their identities (Erikson, 1950). During this time, they tend to orient themselves toward their peers and distance themselves from 
adults (Larson \& Richards, 1991; Steinberg \& Silverberg, 1986), though the shift in orientation toward peers is not unilateral (Berndt, 1979; Smetana, 1988). Unfortunately, most of the studies indicating the shift toward peer orientation used relatively homogeneous participants, excluding ethnic minorities. Research on ethnic identity formation has helped to fill that gap.

Based on the work of Erikson $(1950,1968)$ and Marcia (1980), researchers in the field of ethnic identity formation has shown that ethnic minorities form their identities through stages, much like Erikson (1950) hypothesized for people from the dominant ethnic group. According to Phinney (1988), though, the process for ethnic minorities is slightly different than for those from the majority culture. In general, ethnic minorities go through early stages in which they engage in little exploration or commitment. In a later stage, they explore their identities, but do not commit. In the highest stage, ethnic minorities explore possible identities and commit (Marcia, 1966, 1980; Phinney, 1988).

Though Phinney (1989) showed that ethnic minorities tend to go through these stages similarly, there has been no work on refugee populations such as the Burmese. In addition, I could not find any studies that specifically took into account how students move through these stages in a multicultural setting where they are encouraged to interact with students from a variety of cultural backgrounds.

Students in multicultural classrooms have opportunities to work with each other to access the benefits of the dominant culture. Multicultural education is not a program that can be easily adopted (Gay, 2004). Instead, it is a nuanced philosophy that must be integrated throughout a system (Banks, 1993; Banks \& Banks, 1995; Gay, 2004). 
Though multicultural education needs to be adopted system wide (Daoud, 2003; Sleeter \& Grant, 1987), it is best implemented in the classroom (Lee, 2003).

During adolescence, when students increasingly focus on their peers (Larson \& Richards, 1991; Steinberg \& Silverberg, 1986), being in a multicultural setting grants students the opportunity to interact with peers from a variety of different cultural backgrounds. According to Allport (1954), whose work inspired the field of intergroup contact, interactions between people from different cultural backgrounds can help to reduce prejudice as long as they have equal status, common goals, mutual cooperation, and the contact is supported by authorities. Pettigrew and Tropp (2006) claimed that prejudice reduction still occurred even when all of Allport's (1954) conditions were not met. Many have studied aspects of intergroup contact from specific mediators (e.g., Ata et al., 2009; Binder et al., 2009; Pettigrew et al., 2007) to outcomes other than reduced prejudice (e.g. Leung \& Chiu, 2010; Phinney et al., 2007; Tadmor, et al., 2012). Other researchers have studied intercultural communication, which is focused on linguistic contact between groups (Gudykunst, 2005; Piller, 2011). Based on the work of these researchers (Cheah et al., 2011; Uryu, et al., 2014; Verschueren, 2008; Zimmerman, 1995), it is clear that when minority groups interact with other groups, they become more comfortable in their environments and begin to learn the norms of the dominant society, helping them work toward gaining cultural capital.

Refugee students enter the country especially marginalized and often having experienced significant trauma (McBrien, 2005). According to Ogbu and Simons (1998), refugees have traits of both voluntary and involuntary immigrants. Like voluntary 
immigrants, they chose to leave their country of origin because of fear for their safety (UNHCR, 2005), but they did not necessarily chose to enter the United States, similar to involuntary immigrants. Because they face such major barriers (McBrien, 2005), refugee students often struggle in school. However, McBrien offered several recommendations for schools to help increase the academic achievement of refugee students: (a) help them learn English while retaining their native language, (b) provide social support including connections between home and school, (c) help teachers provide multicultural education for every student and understand the needs of refugee students.

Portes and Zhou (1993) made clear that any recommendations to help refugee students must be tailored to individual refugee populations, as each group has their own specific needs. Community-based and school-wide programs that support students by providing wrap-around services seem to have a positive effect (Block et al., 2014; McBrien \& Ford, 2012; Pinson \& Arnot; 2007; Taylor \& Sidhu, 2012). In the classroom, it is important that teachers connect students' lives to the curriculum (Dooley, 2012). Often refugee students experience marginalization because of differences between their lives and the expectations in the school Dávila (2012). According to Banki (2012) and Kirova (2012), intercultural interactions can help students feel more comfortable and learn the norms of the dominant society. Ferfolja and Vickers (2010) indicated that helping refugee students also has benefits for students from other cultural backgrounds. Though Burmese refugees constitute a relatively small number of students at my school, they are one of the largest groups of refugees admitted to the United States in recent years (Martin \& Yankay, 2012). Unfortunately, a lack of research exists regarding 
the experiences of Burmese students in the United States. Though there are several different ethnic groups that make up Burmese refugees (Fuertes, 2010; Ranard \& Barron, 2007), the Karen have been the focus of the majority of research efforts (Bird et al., 2012; Cross, 1854; Gilhooly \& Lee, 2014; Oh, 2012). Collectively, Burmese refugee students tend to have experienced poor educational environments in their refugee camps (Letchamanan, 2013; Oh, 2012; Purkey, 2006) and feel disconnected from school in the United States (Isik-Ercan, 2012).

Female refugee students are underrepresented in the literature. The little existing research (Harris, 2010; Oikonomidoy, 2007, 2009) indicates female refugee students simultaneously hold on to their past experiences and look toward the future to be successful.

My study focuses on how three female Burmese refugee students experienced a multicultural middle school in the United States, especially their interactions with students from other cultural backgrounds. To explore the experiences of these Burmese refugee students, I selected a qualitative, transcendental phenomenological approach and collected data using interviews, observations, and stimulated recall. In the next section, I describe the literature supporting the approach and data collection methods used for the study.

\section{Review of the Methodological Literature}

To study how Burmese refugee students in my school experienced a multicultural middle school and how they experienced interactions with students from other cultural backgrounds, I used three data collection methods: (a) interview, (b) observation, and 
(c) stimulated recall. All three are qualitative methods, which Creswell (2008) advocated as the most appropriate approach when seeking information from few participants and trying to understand their experiences in depth.

Under the umbrella of qualitative research, Creswell (2013) delineated five approaches: narrative, phenomenology, grounded theory, ethnography, and case study. Though there is overlap among the approaches, they differ in every phase of the research process. Because of the nature of my study, I used a phenomenological approach, which fits well within a constructivist paradigm. Constructivists, according to Guba and Lincoln (2005) view reality as subjective and believe individuals each have their own version of reality. Creswell (2013) stated, "We see the constructivist worldview manifest in phenomenological studies, in which individuals describe their experiences" (p. 25).

According to Creswell (2013), "A phenomenological study describes the common meaning for several individuals of their lived experiences of a concept or a phenomenon" (p. 76). Typically, phenomenological researchers study a single concept or idea with a small group of participants who have experienced that phenomenon. The goal of the researcher is to find the common experiences among the participants within the given phenomenon. Maxwell (2013) laid out two types of phenomenology: hermeneutical and transcendental. In hermeneutical phenomenology, the researcher makes an interpretation of the lived experiences of the participants in the phenomenon. In transcendental phenomenology, the researcher focuses on describing the experiences of the participants and attempts to set aside his or her experiences to provide, "a fresh perspective toward the phenomenon under examination" (p. 80). 
Moustakas (1994) described transcendental phenomenology in great detail. He defined it as, "a scientific study of the appearance of things, of phenomena just as we see them and as they appear to us in consciousness" (p. 49). In other words, transcendental phenomenology is the study of how people experience their perceived environments. According to Moustakas, people experience phenomena through noema and noesis. Noema refers to the thing a person experiences, and noesis refers to how one makes meaning of his or her experience. For a researcher to interpret the experiences of the participants, he or she must make sense of both the noema and noesis and somehow merge the two. In describing the phenomenon, the researcher must make a full textural description of the experiences of the participants. Part of making a textural description is to describe the underlying structures that capture how the participant experiences the world. All along, the goal for the researcher is to achieve epoche, which Moustakas described as the state in which, "we set aside our prejudgments, biases, and preconceived ideas about things" (p. 85). Creswell (2013) made clear that this goal is rarely achieved, but many researchers address epoche by "describing their own experiences with the phenomenon and bracketing out their views before proceeding with the experiences of others" (p. 80). In a sense, the researchers clearly state their biases to the reader so that the reader can understand their interpretations in light of the stated biases.

After bracketing out their experiences, phenomenological researchers write three descriptions of the experiences of the participants: one of the direct experiences, one of their experiences within the context of the phenomenon, and one combining the two into, "the essence of the experience" (Creswell, 2013, p. 80). The combination of the two 
descriptions is the researchers' interpretations of the participants' noema and noesis (Moustakas, 1994).

To conduct the qualitative, transcendental phenomenological study of the experiences of Burmese refugee students in a multicultural middle school classroom, I used interviews, observation, and stimulated recall. In the following sections, I detail the research supporting the use of each method.

\section{Interview}

To study how Burmese refugee students experience multicultural classrooms, I first interviewed them about their experiences. Seidman (2013) stated that interview research seeks to understand "the lived experience of other people and the meaning they make of that experience" (p. 9). It places a high value on the inherent worth of the participants. To understand peoples' experiences, according to Seidman, interviews are necessary.

Vogt, Gardner, and Haeffele (2012) distinguished between exploratory or descriptive and explanatory or confirmatory interviews. Exploratory interviews seek out answers to open-ended questions whereas explanatory interviews test hypotheses or identify causes of specific effects. Rubin and Rubin (2005) echoed this distinction in discussing the designs of interviews. They labeled interviews in which the goal is to explore the experiences of people as cultural and those in which the goal is to get to an explanation of an event as topical. Cultural interviews are typically less structured than topical. Also, in cultural interviews, the factual accuracy of what the interviewee says is 
less important than whether or not the interviewee accurately portrays his or her beliefs; in topical interviews, the ultimate goal is to achieve a factual explanation.

Rubin and Rubin (2005) further categorized interviews as eliciting understanding or meaning or describing events or processes (with a category for those that fall inbetween). They also classified interviews as either narrowly or broadly focused (again, with a third category for those in-between). Using the goals of interviews and scope of focus as axes to a table, they identified nine varieties of interviews: moving from narrowly to broadly focused interviews eliciting understanding are, concept clarification, theory elaboration, and ethnographic interpretation; interviews in between focusing on understanding and events or processes are exit interviews, oral histories and organizational culture research, and life histories; interviews focused on events or processes are investigative interviewing, action research and evaluation research, and elaborated case studies.

When the research questions are exploratory, as mine are, Vogt et al. (2012) recommended in-depth interviews with only a few participants. According to Creswell (2013), in-depth interviews are a common approach for phenomenological studies such as mine. Seidman (2013) called his interview style, "in-depth, phenomenologically based interviewing" (p. 14). In this style, Seidman identified four themes: (a) focusing on the temporary, transitory nature of human experience and the meaning people make of those experiences, (b) seeking out the participants' points of view, (c) helping participants to recreate their lived experiences, and (d) emphasizing the importance of making meaning of experiences. Seidman encouraged researchers to conduct three interviews; the first 
focusing on the life history of the participant, the second focusing on the details of particular experiences, and the third focusing on making meaning of the experiences.

Though he stressed the importance of sticking to the three interview format, Seidman also conceded that interviewing needs to be flexible, because each person and each context is different. The purpose of interviewing in a phenomenological study is to access the experiences of the participants. There are many different ways to do so.

Denzin (1989) delineated three types of interviews according to how the researcher structures them prior to the interview act: schedule standardized, nonscheduled standardized, and non-standardized. Schedule standardized interviews are highly structured; the wording and order of the questions are repeated for each participant. Nonscheduled standardized interviews have a set of information the researcher looks for, but does not structure the wording or order of the questions so that he or she can be more responsive to the interviewees. Non-standardized interviews have no preset questions or required information; they are akin to conversations in which one person is trying to get information from another, but there is no attempt to standardize the information the researcher seeks.

Denzin (1989) evaluated each type of interview according to six criteria: (a) conveying meaning, (b) securing respondents' interest, (c) ensuring the interviewer's clarity, (d) making intentions precise, (e) relating each question to overall intent, and (f) handling the problem of fabrication. He concluded that conveying meaning, securing respondents' interest, and fabrication were problematic with the schedule standardized interview. All of the potential problems that could arise in Denzin's criteria for 
evaluation would be addressed by having well-trained interviewers conduct the other two types of interviews.

A significant difference between the participants and me was our fluency in English. All of the participants were in their school's program for English Language Learners. Fortunately, I drew on several studies as guides for interviewing students who speak other languages.

In interviews with 252 nursing staff, Marshall and While (1994) found that about $20 \%$ spoke English as a second language. For slightly over 5\% $(n=14)$ of the participants, the differences in language ability between the researchers and respondents made analysis difficult. They suggested using semi-structured interviews so that some words could be changed without changing the meaning of the questions, ensuring mutual understanding. Throughout the questioning, they stressed to not lead the interviewees toward particular answers and to let them fully answer questions rather than assuming what they were going to say and finishing answers for them. In addition, they paid attention to their non-verbal messages to clearly show interest and attention toward the participants. To check for understanding, Marshall and While used validating statements after each response. Throughout the analysis stage, Marshall and While emphasized the context in which the interviews occurred to accurately interpret the data.

Some researchers have employed interview methods specifically with Burmese refugees. Watkins, Razee, and Richters (2012) interviewed 59 refugees from Burma (51 were women, all were Karen) regarding their experiences with suffering and distress because of forced emigration and their psychosocial needs due to those stresses. They 
conducted both group and individual interviews, at least some of which were assisted by an interpreter, though they were conducted in English. Watkins et al. went out of their way to ensure participants felt comfortable. All interviews were conducted in settings familiar to the participants and every participant was given a food voucher in gratitude for participating. Most of the participants knew each other because of the small size of the Karen community. According to Watkins et al., the Karen women freely shared their challenges in adjusting to a new society. Participants reported difficulties learning English because of family barriers, unfamiliarity with resources, and differences in cultural norms. They reported confidence in their findings, indicating that they found no reasons why the participants' language ability would have affected the trustworthiness of the findings.

Rosbrook and Schweitzer (2010) also used interviews in studying Burmese refugees. The researchers used semi-structured interviews with nine Karen and Chin adults regarding their stories of migration. Though they did not specifically report on the language used, interpreters were present. It is unclear if the interviews were conducted entirely in English, or in the native languages of the participants. To ensure comfort, they conducted interviews in places familiar to the participants and participants were given a gift voucher to acknowledge their contributions.

In my study, I allowed the participants to choose when and where we met within the school. I began each interview by asking them how their day went to help ensure comfort. I used semi-structured interviews split into three parts to ensure mutual understanding and allow for flexibility. Though I did not provide any incentives for 
participating as some of the above studies did, none of the participants were reluctant to participate and I have no reason to doubt the trustworthiness of their responses.

Even if all participants were to understand each question and provide consistent answers, using only interviews would not be sufficient. Both Creswell (2013) and Maxwell (2013) advocated using multiple methods of data collection in qualitative transcendental phenomenological studies. In addition, Creswell and Maxwell agreed that multiple methods of inquiry increase the likelihood of valid data. According to Vogt et al. (2012), observation is often used in conjunction with other methods. In my study, observation supplements the interviews and provides data for stimulated recall sessions.

\section{Observation}

After interviewing students, I observed the participants in classrooms interacting with students from other cultural backgrounds. According to Vogt et al. (2012), observation is an appropriate research design when the goal of the research is to "glean diverse aspects of lived experience from interaction with others" (p. 68). Bailey (1994) stated it slightly differently in saying that observation works best, "when one wants to study in detail the behavior that occurs in some particular setting or institution" (p. 242). Because I was especially interested in Burmese refugee students' experiences of their interactions with students from other cultures in a multicultural setting, observation was an appropriate choice.

Bailey (1994) identified three major advantages of observational research. First, the researcher can study nonverbal behaviors of students that are otherwise difficult to discern. Second, the research takes place in the natural environment of the participants, 
making it much less artificial than experimental designs. Third, observational research can take place over a long period of time, allowing the researcher to identify trends and patterns of behavior.

Bailey (1994) also identified five disadvantages of observational research. First, the researcher has no control over the environment. However, this could also be seen as a positive in that the research happens in a natural environment. Second, the data cannot be easily quantified. Because I am not seeking to quantify my data, the lack of such will not be a disadvantage. Third, observational studies tend to have small sample sizes, which make them relatively difficult to generalize. My study was not intended to be immediately generalizable, though, so this was not a concern. Fourth, gaining entry to the population a researcher wishes to study can be difficult. I conducted research in the school where I taught, so access was not a problem. Last, people are not typically amenable to being observed regarding sensitive issues because of the lack of anonymity. Though my study may have unearthed some sensitive issues from students, it was not my intention to pry into their personal lives while conducting observations. I only observed their interactions with other students in a classroom, so there were not many opportunities for them to be in sensitive situations. Despite the warnings by Bailey, observation was an appropriate approach to examine how Burmese refugee students interact with students from other cultural backgrounds in my multicultural middle school.

Vogt et al. (2012) specified two types of observational research: naturalistic and participatory. In practice, these represent ends of a continuum rather than distinct types of observation. In participatory research, the researcher actively participates in the field 
he or she studies. In naturalistic research, the researcher passively observes his or her area of study. Bailey (1994) provided the same two types of observational research, but he called them nonparticipant and participant. Nonparticipant observation is akin to naturalistic. Participant observation is akin to participatory.

According to Bailey (1994) and Vogt et al. (2012), each of the two types can be done covertly or overtly. In a covert, naturalistic approach, the researcher does not identify himself and tries to passively observe without impacting those being observed. In an overt, naturalistic approach, the researcher's role is made clear, but he or she remains in the background so as to not influence the participants as much as possible. In the covert, participant approach, the researcher participates in the topic of study and does not reveal himself to be a researcher. In an overt, participatory approach, the researcher makes clear his or her role while still participating in the activity being studied.

Denzin (1989) delineated four slightly different roles of an observational researcher: (a) the complete participant, (b) the participant as observer, (c) the observer as participant, and (d) the complete observer. The complete participant blends in to the group he or she is studying, similar to the covert, participatory approach. For a participant as observer approach, the researcher makes his or her role known while still fully participating in the community, similar to the overt, participatory approach. In the observer as participant approach, the contact between researcher and participants is brief and highly structured. The complete observer approach is most similar to the naturalistic approach in which the researcher does not interact with the participants. Often, this type of observation, according to Denzin, is done through video recording or one-way mirrors. 
Denzin (1989) argued that whatever the approach in observational research, the intent is to "record the ongoing experiences of those observed, through their symbolic world" (p. 157). To do so, the researcher must seek to understand the perspectives of those he or she observes instead of imposing an artificial sense of objectivism. Throughout the process of observation, the researcher must go back and forth between making empirical observations and developing theoretical explanations for the observations. According to Denzin, the process of observing and explaining the observations has several essential elements including continual development and revision of hypotheses, thick and thin descriptions of the observed phenomenon, and understanding the experiences of the participants. I videotaped each observation and later showed the video to the participants and ask them about the events in the observation sessions. This is a method called stimulated recall, which I describe next.

\section{Stimulated Recall}

Stimulated recall was pioneered by Bloom (1953) in his investigation of college students' opinions of lectures and discussion classes. Bloom stated:

The basic idea underlying the method of stimulated recall is that a subject may be enabled to relive an original situation with vividness and accuracy if he is presented with a large number of the cues or stimuli which occurred during the original situation. (p. 161)

In other words, people would be better able to report details of a situation if they were provided cues from the situation. Bloom recorded during five lecture and 29 discussion classes. Within two days, he played the recordings back to students, asking them to 
comment on certain critical points. Students were also allowed to comment on other points they believed to be especially important. Bloom sorted students' comments and made comparisons between lecture and discussion based on them. He found that lecture was better for students' developing knowledge about a topic, whereas discussion was better at developing problem-solving skills.

According to Gass and Mackey (2000), stimulated recall is a flexible research tool that does not require a strict adherence to particular steps. The purpose is to allow participants to reflect upon their experiences during an activity with a support, such as video or audio recording, to help their recall of the activity.

Calderhead (1981) made clear that stimulated recall was not one set of techniques. Instead there are a variety of ways to conduct it. "Typically, it involves the use of audiotapes or videotapes of skilled behaviour, which are used to aid a participant's recall of his thought processes at the time of that behaviour" (p. 212). The cues should help the participant accurately report his or her original thoughts during the behavior. Calderhead stressed that there are several factors, such as anxiety, lack of confidence, distraction, inability to verbalize tacit knowledge, and post hoc justifications of behavior, that could limit the accuracy of participants' reporting. Many of these problems can be checked through internal validity and the degree of match between observed behaviors and reported accounts. Because I gathered data through interviews and observations as well as stimulated recall, I was able to check participants' responses in the stimulated recall sessions against data from the other two methods, increasing the trustworthiness of the data. 
Gaier (1954) specifically examined how to check the validity of participants' reported thoughts through inferences. In his study, 16 college students were divided into five groups. Each group participated in a stimulated recall session of a particular class one, two, four, eight, or 16 days after the recording took place. Students' memories of specific events were highly accurate for the first two days after the recording took place (95\% and 94\%). One could infer, then, that students' memories of their thoughts during those events would be highly accurate as well. Students also reported a high degree of confidence on their memories for the first two days after the recording. Further, students reported thoughts accurate to parts of the process as an idea developed. If they were misrepresenting their thoughts along the way, one would expect participants' reports to be inappropriate for certain parts of the process. Gaier concluded that, though there is no way to immediately ensure the accuracy of participants' reports, stimulated recall could result in more accurate recall of events than without the aid of a memory cue. In my study, I was not necessarily interested in accurate recall of events. I was more interested in the perceptions of experiences in intercultural interactions among Burmese refugee students.

Though the previous studies focused on accuracy of recall, many others have focused on individual's interpretations of events. Lyle (2003) reviewed the strengths and weaknesses of stimulated recall in a study on non-deliberative decision making among sports coaches. Lyle concluded that, though there are limitations, stimulated recall can provide valuable insight into participants' experiences of a situation. 
In a study I described earlier, Uryu et al. (2014) used stimulated recall in their study of a conversation between four educated adults to analyze the sociohistorical reconstructions by the participants of the conversation after it was filmed. They made clear that the stimulated recall sessions did not result in definitive reports of what happened, but rather interpretations based on the participants' social, historical, economic, and contextual experiences. The goal was to capture the participants' interpretations of the conversation, which stimulated recall allowed the researchers to do.

Kagan, Krathwohl, and Miler (1963) developed a specific form of stimulated recall called Interpersonal Process Recall (IPR) in which researchers were able to gain significant insight into participants' processing of earlier events. In this process, Kagan et al. filmed an initial interview and immediately moved participants to another room, with a different interviewer, to conduct the IPR. The new interviewer asked the participant to process the interview in whatever manner he or she saw fit. The initial interviewer was in a different room watching the taped interview as well. Whenever either the participant or original interviewer stopped the tape, it would stop for the other. Kagan et al. found that the change in setting allowed participants, "to reveal at length and in depth much of the subtle or semi-conscious meaning in the interview" (p. 239). Though this exact approach was not be possible in my study (I videotaped an observation rather than an interview, and I was the interviewer for the stimulated recall session), the idea that participants can extensively process and provide insight toward something in which they were previously involved supported my aim in asking students to process 
their experiences in the classroom and provide greater insight than could be captured in interviews and observation alone.

Polio, Gass, and Chapin (2006) investigated how experienced and preservice teachers differed in the feedback they gave to non-native English speakers. The two groups of teachers, on the surface, gave similar feedback to students. When the researchers used stimulated recall to examine the teachers' perceptions of the encounters, they found many differences. Experienced teachers emphasized their roles as teachers, whereas the preservice teachers focused on their personal feelings in the encounters. Experienced teachers focused on students learning language through production, whereas preservice teachers focused on the struggles students were having in production. Last, experienced teachers focused on how well students were comprehending English and what specific problems they were experiencing, whereas the preservice teachers focused on the strategies and procedures they thought might work. Polio et al. stated that stimulated recall allowed them to see, "data that cannot be gleaned from the interaction alone" (p. 258). As a limitation, they pointed out that having the participant stop the tape during the stimulated recall session may have resulted in some important sequences being overlooked. In addition, the open-ended nature of stimulated recall left open the possibility of participants getting off track quite easily. Polio et al., despite the limitations, concluded that stimulated recall allowed them to confirm their inclinations after the interactions, and expand upon them to deepen their findings.

In a study using stimulated recall, Salazar (2012) specifically examined the accounts learners had of corrective feedback experiences. She studied eight Spanish 
students in an English university. Students wrote an assignment, which a teacher corrected with them. Those interactions were filmed and transcribed. The day after the corrective feedback sessions, students participated in a stimulated recall session. Salazar concluded that the combination of corrective feedback and stimulated recall allowed the students to see that they were being corrected and to look for an answer. In addition, the corrections that students noticed in the stimulated recall sessions resulted in later correct usage of those particular grammatical items. Salazar added that stimulated recall seemed to add an element of reflection to students reporting of the initial interaction, meaning that their reports may have not been entirely accurate. However, I did not seek factual reports of events in my study; instead, I sought the participants' experiences of their interactions. The element of reflection Salazar noted was an asset of stimulated recall.

In another study involving reflection by the participants, O'Brien (1993) used stimulated recall to try and understand the thinking that goes on for a marine science researcher and high school biology student. For each participant, O'Brien suggested using multiple videos over several weeks. In addition, he recommended having a neutral third party interviewer conduct the stimulated recall session. Within the session, the participant should self-report as much as possible, rather than the researcher eliciting responses. O'Brien reported success in gaining insight into both participants' thinking through the use of stimulated recall, indicating that it can be used with a wide variety of participants. Many of the studies using stimulated recall used participants older than the participants in my study. O'Brien showed that the method can work with younger participants as well. 
DeWitt and Osborne (2010) supported the idea that young people can successfully conduct stimulated recall sessions in a study with students slightly younger than the Burmese students in my school. They used both video and still photographs with students from ages nine to 11 to elicit information about their experiences with science exhibits at their school. The sessions were done in pairs or groups of three and students were asked what happened in each clip or photo of the science exhibits and how successful the exhibits were. The researchers found that the use of photos or video did not significantly affect students' reports of their experiences; neither photos nor video had significantly better results. DeWitt and Osborne left open the opportunity for students to speak openly about their experiences, allowing them to find the important parts of the experiences. The stimulated recall sessions were conducted approximately one week after the exhibits for the students aged 10 and 11, but 10 to 12 weeks later for the students aged nine and 10. Gaier (1954) stressed the importance of conducting the stimulated recall sessions quickly after the event. However, DeWitt and Osborne (2010) reported clear responses from all participants. Their focus, like of Uryu et al. (2014), was on the experiences of the participants, so perhaps time is not of the essence when factual data is not the goal. I conducted stimulated recall sessions as quickly after observations as possible; but I had to wait several days in most cases. According to DeWitt and Osborne (2010) it should not have negatively affected the quality of the data.

Morgan (2007) also used stimulated recall with students younger than the participants in this study. In total, 12 teachers and 90 students aged three through seven discussed their experiences in learning mathematics, science, and Welsh. Teachers 
filmed their classrooms, and participated in stimulated recall sessions to select video clips to show to the students. Students then participated in stimulated recall session with the investigator within two days after the filmed classroom activity. Morgan found that students typically could recall the activity, but had a difficult time remembering what they were supposed to learn. Also, students focused on the affective aspects of their classroom experiences. When students had more control over choosing which parts of their classroom activities to discuss in stimulated recall sessions, they could remember more of what happened and what they were supposed to be learning. Morgan suggested that for young children, stimulated recall sessions should be conducted shortly after the filmed activities, students should work in pairs during the session, and students should be allowed to select their own parts of the activity to discuss. Morgan indicated that young students can effectively discuss their classroom experiences when given the opportunity during stimulated recall sessions.

O’Brien (1993), DeWitt and Osborne (2010), and Morgan (2007) established that stimulated recall can work with young children. Peterson, Swing, Braverman, and Buss (1982) used stimulated recall with students more similar in age to those in my study (grades five and six). The researchers studied 72 students to examine how their cognitive processes during teaching and learning affected their academic achievement. Students were randomly assigned to one of six classes in which the teachers gave the same lesson using direct instruction. Each lesson was filmed. Immediately following the lessons, the researchers and graduate students conducted stimulated recall sessions with the students. The students watched five segments of the lesson selected by the researchers. 
Researchers conducted the sessions in groups of six, after which they interviewed students individually. Students also took cognitive ability tests based on material from the lesson. Peterson et al. found that, based on student reports, students with higher abilities tended to pay more attention to the lesson, employed a larger variety of cognitive strategies, and acknowledge what the teacher did to help them understand. Peterson et al.'s study showed that young adolescent students can engage effectively in stimulated recall sessions.

Stimulated recall can help students reflect upon their experiences (Polio,Gass, \& Chapin, 2006; 2006; Salazar, 2012). In addition, it seems to work well with both young and adolescent students (O'Brien, 1993; Peterson et al., 1982). In a transcendental phenomenological study, the goal of the researcher is to make sense of the participants' noema (the things they experience) and noesis (their interpretations of their experiences) (Moustakas, 1994). Stimulated recall allows a researcher to explore participants' noesis by having them reflect upon their experiences.

\section{Summary of Methodological Review}

In the methodological review, I articulated my rationale for using qualitative study to explore the experiences of Burmese refugee students in a multicultural middle school. I selected a qualitative approach because it is appropriate when the researcher seeks information from a few participants and tries to understand their experiences in depth (Creswell, 2008). More specifically, I used a transcendental phenomenological approach (Creswell, 2013; Moustakas, 1994), in which I described the experiences of the participants while setting aside my own biases and experiences. 
Transcendental phenomenological researchers typically employ multiple methods, including interview and observation (Creswell, 2013). In my study, I used three data collection methods: interviews, observations, and stimulated recall. Interviews were most appropriate for understanding the experiences of the participants (Seidman, 2013). Observations helped me to record the experiences of the participants in a natural setting (Denzin, 1989). After the observation sessions, which were video recorded (a common practice according to Denzin [1989]), I used the videos to conduct stimulated recall sessions with the participants, which allowed them to re-experience and reflect upon a particular situation through the stimulus of the video (Bloom, 1953).

The first step in my process was to conduct interviews, which were exploratory in nature (Vogt, Gardner, \& Haeffele, 2012). Creswell (2013), Seidman (2013), and Vogt et al. (2012) recommended in-depth interviews with a few participants. I use open-ended questions in which I searched for similar information but did not have a strict question format, which Denzin (1989) called nonscheduled standardized interviewing and Barriball and While (1994) called semi-structured interviewing. According to Barriball and While, this approach builds in validity because it elicits the perceptions of each participant while somewhat controlling for misunderstandings of each question.

Then, I observed students during intercultural interactions in their classes as an overt, naturalistic observer (Bailey, 1994; Vogt et al., 2012). During the observation sessions, I video recorded the students engaged in intercultural interactions.

Last, I used the video recorded observations to conduct stimulated recall sessions with participants. In stimulated recall, participants watch a video of themselves engaged 
in an activity and comment on it. According to Calderhead (1981) and Gass and Mackey (2000), there are no set steps for stimulated recall. Instead, it is a method intended for participants to reflect upon their experiences. Among others, Lyle (2003) made clear that stimulated recall is especially good at providing insight into participants' experiences of a situation.

The three data collection methods combined to provide a vivid picture of the experiences of Burmese refugee students in a multicultural middle school. In the next chapter, I describe the participants in my study, detail the procedures I used to examine their experiences, discuss my role as a researcher, explain how I collected and analyzed the data, outline limitations of the study, and describe how I established validity. 


\section{Chapter 3: Methods}

In Chapter 1, I described many of the challenges refugee students face in middle school, highlighting intercultural interaction as a way students could start to overcome their challenges. In my middle school, Burmese refugee students tend to not interact with many students who are from different cultural backgrounds. Their lack of intercultural interactions may be keeping them from learning the specific habitus to be successful in middle school and gain cultural capital. I used three research questions to study this problem:

1. What were the experiences of Burmese refugees in multicultural middle school classrooms?

2. How did Burmese refugee students experience interactions with students from other cultural backgrounds?

3. How did the interactions Burmese refugee students have with students from other cultural backgrounds affect their school experiences, including learning and identity formation?

In Chapter 2, I detailed the research literature having to do with refugee students in middle school classrooms. I explored the experiences of Burmese refugee students in multicultural middle school classrooms through the lens of Bourdieu's theory of field and theory of social and cultural reproduction (Bourdieu, 1973, 1977, 1990; Bourdieu \& Wacquant, 1992), in which people are successful because their dispositions match the successful habitus within a field. Their success gives them cultural capital, which reinforces successful habitus that match their dispositions. Middle school is an especially 
important time in which students can gain cultural capital because their social lives hold great importance for them. In middle school, adolescents work through a stage of identify formation (Erikson, 1950, 1968) in which they tend to shift orientations from their parents to their peers (Larson \& Richards, 1991; Steinberg \& Silverberg, 1986). For ethnic minorities, their identity formation includes a process of finding their ethnic identities (Marcia, 1966, 1980; Phinney, 1988).

Because my study was in a multicultural context, I also reviewed the literature regarding multicultural education. Multicultural education is an idea that must be integrated into schools rather than imposed upon (Banks, 1993; Banks \& Banks, 1995; Gay, 2004). Though it is best to adopt system-wide (Daoud, 2003; Sleeter \& Grant, 1987), the primary point of implementation for multicultural education is in the classroom (Lee, 2003).

In such multicultural contexts, students have ample opportunities for intercultural interaction, which help in a variety of ways (Alfassi, 2009; den Brok et al., 2006; Gersten \& Baker, 2000; Gurin et al., 2011; Janzen, 2008; McBrien, 2005; Piccolo et al., 2008; Pugh et al., 2012; Taylor \& Sidhu, 2012; Stoughton \& Sivertson, 2005). Intercultural interactions can reduce prejudice (Allport, 1954; Pettigrew \& Tropp, 2006), lead to more increased comfort and familiarity with the norms of the hegemonic culture (Cheah et al., 2011; Uryu et al., 2014; Verschueren, 2008; Zimmerman, 1995).

Refugee students, in particular, stand to benefit from intercultural interaction due to their struggles (McBrien, 2005) in being resettled. Though often discussed together, refugees are distinct from other immigrants (Cheah et al., 2011; Ogbu \& Simons, 1998; 
Taylor \& Sidhu, 2012; UNHCR, 2005; Valenta, 2010). Researchers have examined supports that help refugee students, finding that holistic services seem to help (Block et al., 2014; McBrien \& Ford, 2012; Pinson \& Arnot; 2007; Taylor \& Sidhu, 2012). Like multicultural education, a key point of implementation of supports for refugee students is the classroom (Banki, 2012; Dávila, 2012; Dooley, 2012; Kirova, 2012). Female refugees in particular seem to bridge their past experiences by looking toward the future to be successful in school (Harris, 2010; Oikonomidoy, 2007, 2009).

One of the largest refugee groups to enter the United States recently is from Burma (Martin \& Yankay, 2012; UNHCR, 2015). Burmese refugees have tended to come from inadequate educational settings (Letchamanan, 2013; Oh, 2012; Purkey, 2006) and feel disconnected to school in the United States (Iski-Ercan, 2012).

I also explained the methods I used to investigate my research questions in Chapter 2. Specifically, I used a qualitative, transcendental phenomenological approach (Creswell, 2008, 2013; Moustakas, 1994). I conducted semi-structured interviews (Barriball \& While, 1994), which are an effective method for exploring the subjective experiences of participants in a study, especially to ensure mutual understanding of those who speak languages other than mine (Marshall \& While 1994), observed them engaged in intercultural interactions in the classroom using an overt, naturalistic approach (Bailey, 1994; Vogt et al., 2012), then conducted stimulated recall sessions (Calderhead, 1981; Gass \& Mackey, 2000) with the participants to allow them to share their understandings of their experiences (DeWitt \& Osborne, 2010; Salazar, 2012). 
In this chapter, I describe in more detail the methods I used to study how Burmese refugee students experienced multicultural classrooms in my middle school, how they experienced interactions with students from other cultural backgrounds, and how their interactions with other students shaped their school experience.

\section{Research Methods}

Because I explored the experiences of a small number of Burmese refugee students in middle school, qualitative research methods were most appropriate. According to Creswell (2008), qualitative methods are most appropriate when the researcher is seeking to learn about the views of individuals or obtain detailed information about a few people. Krathwohl (2009) differentiated between quantitative and qualitative studies in four ways:

- Qualitative studies explore, describe, or explain phenomena, whereas quantitative studies explain, corroborate, predict, or generally explore phenomena.

- Qualitative studies are inductive whereas quantitative studies are deductive.

- Data for qualitative studies is typically in the form of words, whereas in quantitative studies, data is in the form of numbers and measures.

- Examining a phenomenon holistically is a trait of qualitative studies; quantitative studies typically break the phenomenon apart to study its parts. The components of my study all fit well into Krathwohl's description of qualitative research. I inductively explored the holistic experiences of Burmese refugee students in a multicultural middle school using their own words. 
Creswell (2013) echoed Krathwohl's (2009) assertion that qualitative research designs are best for exploring a topic in depth. Creswell (2013) stated, "Qualitative researchers use an emerging qualitative approach to inquiry" and collect data "in a natural setting sensitive to the people and places under study" (p. 44). I studied one topic in depth, which Creswell stressed as one of the main characteristics of a good qualitative study. In addition, each of my research questions was open-ended and required a variety of data sources, both hallmarks of good qualitative studies. Creswell also emphasized multiple sources of data collection; I used interviews, observations, and stimulated recall to gather my data.

In seeking information from Burmese refugee students about their experiences in middle school, I encouraged them to speak their truths and I accepted their perspectives as inherently accurate. This is indicative of a paradigm Creswell (2009) called social constructivist and Guba and Lincoln (2005) called constructivist. Previously, Lincoln and Guba (1985) had named the paradigm naturalistic. Creswell (2013) stated that this same paradigm (or what he called a worldview) was often known as interpretivism. For consistency, I use the term constructivist.

The constructivist paradigm centers around the multiplicity of views participants have. To constructivists, reality is subjective; individuals construct reality while trying to understand the world they inhabit (Guba \& Lincoln, 2005). Studies rooted in a naturalistic paradigm seek to understand the experiences of the participants (Creswell, 2013). Researchers interpret the experiences of the participants while positioning themselves based on their own experiences. According to Creswell (2013), "the 
researcher's intent... is to make sense of (or interpret) the meanings others have about the world" (p. 25). My major goal in this research project was to understand the experiences Burmese refugee students have in a multicultural middle school. Because I emphasized the individual experiences of the participants, this approach fits well within the constructivist paradigm.

Under the umbrella of constructivism, there are many possible approaches to a qualitative study. I took a transcendental phenomenological approach, which is a specific form of one of the five qualitative approaches Creswell (2013) delineated: (a) narrative, (b) phenomenology, (c) grounded theory, (d) ethnography, and (e) case study. In transcendental phenomenology, researchers study the lived experiences of a few people and bracket out their experiences and biases to most effectively report the experiences of the participants (Moustakas, 1994).

According to Creswell (2013), researchers in a phenomenological study often gather data through in-depth interviews, observations, journals, and other procedures to understand the experiences of the participants. I used interviews, observations, and stimulated recall to gather my data. Each method allowed me to access the experiences of Burmese refugee students in multicultural classrooms. Participants could openly share their experiences in interviews. I could see directly how the participants interact with other students during observations. Participants could reflect upon their intercultural interactions [which Moustakas (1994) stated was an important part of a transcendental phenomenological study] and further share their experiences during stimulated recall sessions. 
In gathering my data, I followed a general guide, but remained flexible. Maxwell (2013) suggested qualitative researchers enter into a research project with flexibility built in to their research questions. He argued that settling on specific, inflexible research questions too early in a qualitative study could result in, "a Type III error-answering the wrong question" (p. 73). Instead, Maxwell advocated having preliminary research questions that grow more focused throughout the process of gathering and analyzing data. Throughout the process, the methods need to be a good fit for the research questions and information the researcher wants to gather. The methods can be highly structured or not. My approach was somewhere in between. For each research question, I used several methods to gather data. I remained flexible, though, on the amount of time needed for those methods as well as which methods I will rely on more heavily than others. As the study proceeded, I found that I needed more time to complete the observations than I had anticipated. Even though I needed more time in observation, I needed less time in stimulated recall than I anticipated. Overall, I spent approximately the same amount of time with each participant in each phase of the study, but did not keep a strict accounting of how long I spent with each during each part of the study.

For my first research question (What were the experiences of Burmese refugees in multicultural middle school classrooms?), I gathered data using in-depth interviews, and supplemented with observation and stimulated recall. For my second research question (How did Burmese refugee students experience interactions with students from other cultural backgrounds?), I primarily used stimulated recall, supplemented with observation and interviews. For my third research question (How did the interactions 
Burmese refugee students have with students from other cultural backgrounds affect their school experiences, including learning and identity formation?), I used a combination of stimulated recall, interviews, and observation (see Table 2).

Table 2

Primary and Secondary Methods for Addressing Each Research Question

Research Method

Research Question

Interview Observation Stimulated Recall

What were the experiences of Burmese refugee students in Primary Secondary $\quad$ Secondary multicultural middle school classrooms?

How did Burmese refugee students experience interactions with students from other Secondary Secondary Primary cultural backgrounds?

How did the interactions

Burmese refugee students have with students from other cultural backgrounds affect their school Primary Primary Primary experiences, including learning and identity formation? 


\section{Participants}

Three Burmese refugee students were the participants in this study. I interviewed, observed, and conducted stimulated recall sessions with these three Burmese refugee students in seventh and eighth grade who returned both their permission forms.

\section{Sampling}

Krathwohl (2009) said that any time researchers choose participants to represent a larger population they are engaged in sampling. Though I did not necessarily try to access data that could be generalized, I chose participants who might display tendencies indicative of a larger population. Krathwohl distinguished between two types of sampling: probability and nonprobability. Probability sampling involves random sampling, whereas nonprobability sampling does not. Because I did not use random sampling, the selection of my participants was an example of Krathwohl's nonprobability sampling.

More specifically, the selection of the participants in my study was an example of convenience sampling (Creswell, 2013). In convenience sampling, the researcher chooses participants based on who he has access to. Maxwell (2013) reported that many authors have said to avoid convenience sampling. However, Maxwell also argued the term convenience sampling is associated with quantitative research and inappropriate for qualitative research. Instead, he advocated the use of the term purposeful selection.

Creswell (2013) used the term purposeful sampling as an umbrella term to capture several different types of sampling procedures. To him, purposeful sampling referred to the process by which a researcher chooses, "individuals and sites for study because they 
can purposefully inform an understanding of the research problem and central phenomenon in the study" (p. 156). Though my actual participants were chosen out of convenience, I was purposeful in choosing representatives of a relatively small groupBurmese refugee students in a multicultural middle school. As such, convenience sampling, or purposeful sampling, is the only viable option I had.

Throughout the study conducted in the spring of the 2014-2015 school year, I was employed as a social studies teacher in the building where the research occurred. So as to not conflate my dual roles as teacher and researcher, which Seidman (2013) specifically said to avoid when conducting interviews, I chose participants based on who I was able to observe while I was not teaching during the school day (my preparation periods). I identified four possible Burmese refugees who had math, science, or social studies during my preparation periods. Three of the potential participants returned their assent and consent forms: Ohma, Khalidah, and Shamshidah (pseudonyms). Two of the students were in seventh grade (Ohma and Shamshidah); one was in eighth grade (Khalidah). Two of the students identified as Rohingya (Khalidah and Shamshidah); one identified as Burmese (Ohma). All three were female and identified as Muslim. I was the social studies teacher for two of the participants (Ohma and Shamshidah) during the study, and I had been the social studies teacher for Khalidah the previous school year (2013-2014).

\section{Ohma}

Ohma identified as Burmese and was in seventh grade. She was in English language development (ELD) level four. In my experience, students in ELD level four tend to be nearly fluent speakers who do not quite have a full grasp of academic English. 
In a school such as mine, where almost all students qualify for free or reduced lunch, students who are in ELD level four seem to perform within the range of most mainstream students. She was born in a refugee camp in Thailand, where she lived until moving to Texas, then Oregon. Ohma was relatively social, but with a small group of friends. She was the least connected to her family, but did not report having a wide circle of friends. I observed her in her science class, which was the last class of the day.

Ohma was a quiet student who did well in school. She was a hard worker who helped others. She responded strongly to humor in the classroom. Ohma disliked when other students misbehaved, but admitted that she also might misbehave if put in groups with her friends who were off task. She described herself as a "villager," indicating she viewed herself as somehow different from others.

Ohma was involved in the Schools Uniting Neighborhoods program at her school. At the time of the study, she was on the soccer team, which was run through Schools Uniting Neighborhoods. In previous terms she had been involved in several other classes and programs. One of the groups she had previously been a part of was a class specifically for Burmese refugee students. In the class, adults from Burma worked with students to assist them with study skills and homework. Unfortunately, there was no direct line of communication between that particular after-school program and teachers at the school. 


\section{Khalidah}

Khalidah identified as Rohingya and was in eighth grade. She was in ELD level five, also called monitored. In this level, students tend to be relatively fluent in English, needing just a little support to be able to fully grasp academic language. She was born in Bangladesh, where she lived until she moved to Texas before settling in Oregon. During some of this time, Khalidah lived with Ohma. In school, I observed Khalidah in her social studies class, which was also her last class of the day.

Khalidah was the most outgoing of the participants; she reported having more friends than the other participants while still being somewhat connected to her family. She enjoyed having fun in class and was very focused on relationships with others. I saw her work seamlessly with more students than the other participants. She oriented herself to her family, who she enjoyed spending time with. She strongly identified as Muslim. She enjoyed Islamic school, even though she disliked the techniques the teacher used. She liked school in the U.S. equally to school in Bangladesh, enjoying the singing and activities in Bangladesh, but she had more friends in the United States.

During the study, Khalidah was not involved in after-school activities, but had previously been very involved in the Schools Uniting Neighborhoods program. Like Ohma, she was previously involved in a group specifically for Burmese refugee students.

\section{Shamshidah}

Shamshidah also identified as Rohingya and was in seventh grade. She was born in Thailand and later moved to Malaysia, where she lived until she moved to the United States. Shamshidah was in ELD level three. In my experience, students in ELD level 
three have struggled to effectively communicate their ideas, especially in writing. However, they typically have been able to comprehend the material presented in a class, especially when the teacher is aware of their language abilities. In addition, they have been able to effectively communicate what they are thinking, while using a somewhat limited vocabulary.

Shamshidah was very hard working and typically outperformed other ELD level three students that I have taught. In school, she was far less social than the other two participants; I rarely saw her socializing with others, and she strongly preferred to work by herself. She reported that she spent most of her time outside of school with her family. I observed Shamshidah in her math class, which was at the end of the day.

Shamshidah was very shy. In her interviews, she was so quiet the audiorecorder hardly picked up her voice. She was in English Language Development level three, still learning conversational English. As a result, she frequently paused while she planned her words. She identified herself as shy, quiet, nervous, and feeling isolated. She strongly identified with her family and Islam. She wanted to do well in school so she could have a good career. Shamshidah, unlike Participants 1 and 2, was not involved in after-school activities.

Maxwell (2013) stressed the importance of building relationships with the participants in a study. Because all three participants had me as a teacher (two during the study and one prior to the study), I had a relationship with them. In addition I had previously conducted a study in which I interviewed the older sister of Khalidah, and had met her prior to being her teacher during the 2013-2014 school year. Long before the 
study, Ohma had lived with Khalidah and was familiar with me via the interview I had done with the sister of Khalidah.

According to Maxwell, the relationships must be managed continuously; simple access is not enough when the researcher is the instrument of the research. In addition, Maxwell argued the researcher must build specific kinds of rapport with the participants. In discussing how to choose participants to interview, Seidman (2013) cautioned against teachers interviewing their own students. He reasoned that students cannot be fully open with their teacher because of the power teachers wield. His example, however, was about exploring a teaching program or curriculum. I investigated Burmese refugee students' experiences in school. If I built appropriate rapport and continually paid attention to the relationships I formed with students, as advocated by Maxwell (2013), I should have been able to access the information I was seeking to answer my research questions. As a caution, I chose to study students outside of my own classroom and made clear to them that my role as researcher was separate from my role as a teacher. The relationships I built prior to and during this study were complex because of the dual role I played as both teacher and researcher. I worked very hard with the participants to keep my roles separate; I never discussed the study while I was in the role of their teacher, and I always tried to make clear to them what role I was occupying while I talked with them.

Maxwell (2013) emphasized trying to understand the perspectives and perceptions of the participants in a qualitative study, especially those from different cultural backgrounds than the researcher. Maxwell provided an example of students interviewing others without anticipating problems. The interviews were uncomfortable and 
unproductive because the participants experienced the interviews very differently than the students. Even though seeking to understand the experiences of Burmese refugee students is inherent in the design of my study, I tried to remain aware of any differing experiences throughout the research process. I attempted to anticipate how they might experience the research process and adjusted my approach so I could strengthen my relationship with them as well as gather data that addresses my research questions.

In this section, I discussed the participants in my study. In the next section, I detail the procedures I used to examine their experiences in multicultural middle school classrooms.

\section{Procedures}

Qualitative studies use multiple sources of data (Creswell, 2013; Maxwell, 2013). According to Maxwell (2013), using multiple methods strengthens the validity of a study through triangulation and allows for a complex understanding of the various aspects of the phenomenon being studied.

I used a combination of interviews, observation, and stimulated recall. For the interviews and observations, I used protocols (see Appendices A \& B) to keep myself focused on gathering appropriate data, but I also remained flexible. Specifically speaking of interviews, Denzin (1989) and Barriball and While (1994) indicated using semistructured designs allowed for the researcher to stay responsive to the participants while still maintaining focus on gathering useful data. Allowing for flexibility was important in observational research as well, as I watched for intercultural interactions involving Burmese refugee students, but I wanted to minimally influence the interactions 
themselves. I remained flexible in the stimulated recall sessions by encouraging the participants to discuss whatever they found interesting rather than me dictating which parts were most important.

Maxwell (2013) stressed that multiple methods should be used when they can complement each other. If different methods result in similar data and have similar issues with validity, there is no point to using them. In the interview portion of my study, I examined the overall perceptions of Burmese refugee students in my school. In the observation portion, I looked at how Burmese refugee students interact with students from other cultural backgrounds. In the stimulated recall portion, I studied Burmese refugee students' reactions to their interactions to support or compare to the data I gathered through the other two methods.

\section{Interviews}

My first step in exploring the experiences of Burmese refugee students in a multicultural middle school was to interview them. According to Vogt et al. (2012), interviews are most effective "when you are seeking knowledge that is best obtained from members of your target population because it is subjective or internal to the people interviewed" (p. 36). Rubin and Rubin (2005) suggested using interviews when a researcher was seeking information requiring people to describe their experiences. I chose interview as a method to obtain subjective, internal, explanations of the participants' experiences. I sought to find out how female Burmese refugee students experienced schools in the United States, how they interacted with students from other cultures, and how they perceived those interactions shaping their experiences. 
I asked open-ended questions to elicit the perceived experiences of the Burmese refugee students in multicultural classrooms (see Appendix A). According to the framework presented by Vogt et al. (2012), the interview portion of my research was exploratory. Narrowing more, Rubin and Rubin's (2005) presented a framework in which my study would be classified as ethnographic investigation or theory elaboration. I followed a semi-structured guide of questions to address the topics I wanted to explore. I remained flexible, however, in conducting the interviews so I could adjust as necessary according to the answers provided by the participants. According to Denzin's (1989) framework, my interviews were nonscheduled standardized, otherwise known as semi-structured (Barriball \& While, 1994; Seidman, 2013). One of the reasons I needed to stay flexible was that all of the participants were English Language Learners (Marshall \& While, 1994). Rosbrook and Schweitzer (2010) and Watkins et al. (2012) made clear that interviews could be productive with participants who were learning English if comfort was ensured and appropriate steps were taken to make sure they understood the questions and responded appropriately. I was careful to follow the recommendations of Marshall and While (1994) in using semi-structured interviews; I did not use leading questions, paid attention to non-verbal messages, used validating statements, and recorded the context of the interviews to report in the results section of this paper.

I divided the interview protocol into three parts (see Appendix A), as recommended by Seidman (2013), so as to keep the participants as comfortable as possible, allow them to focus on one general topic at a time, give them appropriate time to answer each set of questions, and ensure time for clarification if necessary. Part one of 
the interview protocol focused on the participants' lives outside of school and their experiences prior to entering the United States. Part two focused on the participants' experiences in school in the United States. Part three focused on the participants' experiences in working with other students in their classes. By conducting the interviews in three parts, I was able to find mutually agreeable times to interview the students. Because I conducted the interviews before school, during lunch, and after school, each section had to be short enough to not interrupt the participants' other scheduled activities or classes. Each section lasted approximately 20 minutes, so I was able to complete each section during small windows of time. Within each section, there were few enough questions that the participants could effectively elaborate without going over constrained time. The few questions also gave me time to clarify any answers I did not understand to ensure their meanings were accurately communicated.

I audio recorded each interview and took notes to aid in my understanding. I transcribed each interview and gave a copy to each participant for member checking. Member checking gave each participant an opportunity to read their own words and verify what they meant to say. I transcribed each interview prior to conducting the next section with each participant so that I could clarify anything from the previous section and so that the data could inform the next section.

As informative as interviews can be, especially when appropriate steps are taken to ensure mutual understanding, Creswell (2013) and Maxwell (2013) stated that using multiple methods strengthens the quality of a study by allowing the researcher to check 
his or her conclusions against data from another method. To supplement the interview data, I used both observation and stimulated recall.

\section{Observations}

After interviews, I observed the participants in their classrooms, paying special attention to their interactions with students from other cultural backgrounds. I primarily used an overt, naturalistic approach (Bailey, 1994; Vogt et al., 2012) in which I was a passive observer in the classroom, and made my role as a researcher clear to the participants and other students in the class. My observations supplemented the data I gathered through interviews. In the observations, I sought to find discrepancies or corroboration in what participants reported in interviews and how they behaved in a classroom. Vogt et al. (2012) stressed that observational designs are rarely used in isolation, and that interviews are a good fit as a source of corroboration. Bailey (1994) also stated that observation is typically used in conjunction with other methods of collecting data.

My approach was most similar to Bailey's (1994) and Vogt et al.'s (2012) overt, naturalistic approach or Denzin's (1989) complete observer approach, though it had elements of the observer as participant approach. It was overt because I made my role as a researcher clear to the participants and other students in the classroom. My role was naturalistic because, even though I was a teacher in the school, I was a passive observer during observation sessions.

Prior to observation sessions, I talked with the teachers to ensure there would be ample opportunities for the participants to engage in intercultural interaction during the 
regular course of classroom activities. With each participant, I conducted two or three informal observation sessions without filming, but watched how the participants interacted with other students and, more importantly, made myself familiar to the class so that they were used to my presence. After the informal sessions, I conducted three to four formal observation sessions with each participant, during which I filmed intercultural interactions for use in the stimulated recall sessions. These participants were accustomed to being filmed in their classroom because in this school, teachers routinely filmed their classes for their own professional growth. As much as possible, I filmed the participants working with students from a variety of different cultural backgrounds. I took field notes during the observation sessions so I could fully and accurately report the context in which the interactions occurred. I used a rough guide (Appendix B) to structure my observations and field notes.

The observation portions of my research were informed by the interviews and led to further reflection by the participants in the stimulated recall portion. As Maxwell (2013) stated, using multiple methods strengthens the quality of the research. Further, observational research is often supplemented by other approaches (Vogt et al., 2012). To explore the experiences Burmese refugee students have in their interactions with students from other cultural backgrounds, I followed my observations with stimulated recall sessions.

\section{Stimulated Recall}

I triangulated the data gathered from interviews and observations by conducting stimulated recall sessions with the participants, which allowed me to explore students' 
perceptions of specific experiences in a middle school classroom (the videotaped observations) deeply. The sessions also allowed the participants to reflect upon their experiences, which Moustakas (1994) called noesis, and stressed as an important part of a transcendental phenomenological study.

I used stimulated recall as a combination of observation and interview in which participants engaged in particular activities (interacting with students from other cultural backgrounds) that I observed and filmed. Shortly after the filmed activity, each participant then viewed the recorded activity while I was present. I used the video to prompt the participants to explain their thinking during the intercultural interactions (Bloom, 1953; Gass \& Mackey, 2000). Though I used a semi-structured protocol for both interviewing and observing students, the stimulated recall sessions were unscripted to allow participants to share their thinking freely. I let the participants comment on parts they thought were important, pausing the video when they began commenting. Many times, though, I had to pause the video at the end of an interaction and ask the participants about their interpretations of the interaction.

Bloom (1953) pioneered stimulated recall in a study on college students' opinions regarding lecture and discussion classes. While I used a similar approach, there were some important differences between how Bloom (1953) used stimulated recall and how I used it. First and foremost, the populations differ; Bloom studied college students and I studied students in middle school. Second, Bloom asked students to comment on points he thought were important (though he did leave room for them to spontaneously 
comment); I primarily let students comment on what they found important and only focused on other times after they had watched a section without comment.

There were some other differences between what researchers have stated about stimulated recall and how I implemented it. Before discussing those differences, it is important to note that, as Calderhead (1981) and Gass and Mackey (2000) stated, stimulated recall is not a specific set of techniques with a roadmap for its use. Instead, it is a guide in which researchers provide memory cues to ask participants about their experiences in a particular event.

One difference between how I conducted stimulated recall and the recommendations of some researchers was the time elapsed between the filmed activity and the stimulated recall session. Bloom (1953) and Gaier (1954) both stressed the importance of conducting the stimulated recall session within two days of the event about which the participants will process. However, other researchers (e.g. DeWitt \& Osborne, 2010) had success conducing the sessions more than one week after the observed event. In my study, more than one week elapsed between the observations and stimulated recall sessions for almost every case. Researchers have also debated the type of data collected in stimulated recall sessions. Researchers such as Bloom (1953), Calderhead (1981), and Polio et al. (2006) noted issues with the accuracy of participants' memories when asking them to process events through a process such as stimulated recall. Others (e.g. Kagan et al., 1963; Uryu et al., 2014) pointed out; however, the goal of some stimulated recall sessions is to capture the participants' reactions to their earlier experiences, not 
necessarily to ask them to accurately report what happened. I sought the participants' interpretations of their experiences in intercultural interaction.

In my research, I asked participants to reflect upon the filmed observations and report their reactions on important parts of the conversation. I sought their interpretations of their experiences. To best capture what parts of the interactions students thought were important, I asked them to stop the videotape when they thought something important happened, rather than me deciding on the important parts of the interaction. Polio et al. (2006) warned that this approach may leave some particularly important sections from the perspective of the researcher ignored. I tried to temper this by pointing out parts of the video I thought were important only after the students had finished watching a section without comment.

I showed the filmed observations to the participants using my computer. As we watched and commented, I used a screen capture function to audio record our comments synced with what was happening on my computer screen. This process resulted in a new video of each observation session with our comments embedded into the video.

Conducting stimulated recall sessions after interviews and observations allowed me to explore the participants' noesis, or interpretation of their experiences (Moustakas, 1994). It also allowed me reassess previously gathered data (Krathwohl, 2009) to aid in establishing validity (Creswell, 2013; Maxwell, 2013). Next, I describe in detail the instruments that aided me in gathering data, my role as the researcher, how I analyzed the data, and how I established validity. 


\section{Instruments and Measures}

Due to the qualitative nature of my study, I tried to be responsive to my participants throughout the process. However, I relied on some guides to ensure the data would address my research questions. I used semi-structured protocols for the interview (Appendix A) and observation (Appendix B) portions of my study. For the stimulated recall portion, I asked the participants to comment on anything they thought was important, letting them guide the session as much as possible. I only directly asked them about their experiences after they had the opportunity to watch and comment on an entire section.

For each interview, I used a semi-structured interview protocol (see Appendix A) as a guide. As recommended by Seidman (2013), I conducted three interviews with each participant; each interview was informed by the data from the previous interview and had a slightly different focus. The first section focused on their home lives and their experiences in their countries of origin. The second section focused on their experiences in school in the United States. The third section focused on their experiences interacting with students from other cultural backgrounds.

The interview protocols were semi-structured (Barriball \& While, 1994; Seidman, 2013) to maintain consistency between each participant, while allowing for flexibility. This was especially important because the participants were all non-native English speakers in their school's program for English language learners (Marshall \& While, 1994). Vogt et al. (2012) recommended having several different protocols available to be prepared for participants who are comfortable with very open-ended questions as well as 
participants who require more structure to elicit data. My approach was to have several open-ended questions as well as several more focused questions so that I could elicit the most useful data from each participant.

I audio recorded each interview while also taking notes to aid in understanding. I transcribed each interview prior to the next one so I could clarify any misunderstandings and use the data to inform the next section. As I completed the transcriptions, I gave a copy to each participant for member checking.

Like the interviews, I used an observation protocol (see Appendix B) to focus my data-gathering. Vogt et al. (2012) stated that observation can be improvisational, but can be guided by the research questions. The observation protocol focused on observing the interactions between Burmese refugee students and students from other cultural backgrounds. I took into account the context in which the interactions occurred, the content of the interactions, and verbal and non-verbal reactions by the participants.

I filmed each observation session for use in stimulated recall sessions. Prior to filming, I spent time in each participant's classroom to familiarize myself with the classroom layout and atmosphere as well as to let the students in the class get used to my presence. I used my mobile phone to film so I could be as unobtrusive as possible. I also filmed more than just the observation sessions, so the participants and other students in the class would not know when I was actually recording. Any filming that was not used for observation and stimulated recall was immediately deleted.

For the stimulated recall sessions, I used video of the observation sessions, which had observation protocols. My primary approach in using stimulated recall was to ask 
students to identify important parts of the videotaped sessions. If students were unable to identify important parts, I asked them to talk about parts I thought were important. I did this by watching one section of filmed observation at a time. If the participant began to comment, I would pause the video and let them comment. During these sessions, I used a screen capture function that audio recorded what we were saying and synced our comments with our actions on my computer screen, such as pausing and rewinding. After each stimulated recall session, I had a video of our comments with what we did on the screen during the session.

Throughout the data-gathering process, I had to remain cognizant of my dual role as teacher and researcher, even though I was not actively the students' teacher during data gathering. In the next section, I describe how I addressed this issue and other ways I established validity.

\section{Establishing Validity}

According to Maxwell (2013), methods do not ensure validity; only evidence can make validity threats implausible. Researchers make arguments based on their evidence and other academics infer from the evidence that the conclusions are valid. Creswell (2013) and Maxwell (2013) named many strategies to increase the chances of gaining valid evidence. In my study, I incorporated several of their suggestions. First, I had long-term engagement with my participants, as I was their teacher prior to starting the study. Also, I have been working with the Burmese refugee community for the last three years, so many of the families were familiar with me. Throughout the study, I spent a great deal of time with the students, getting to know them well. In addition, I used 
triangulation, or multiple sources of data-- interviews, observation, and stimulated recall - to corroborate the evidence I gathered from each. Because a qualitative inquiry is iterative (Krathwohl, 2009), I continually reassessed previously gathered data in light of more recent evidence.

To avoid problems with validity in interviewing, Denzin (1989) recommended the interviewer clearly state his or her role in the process and present him or herself consistently. In addition, the relationship between the interviewer and interviewee should be clear and mutually understood. Seidman (2013) said the researcher should conduct several interviews spread over a period of time, which has two benefits for validity. First, statements by the participants can be checked against previous statements to ensure internal validity. Also, using several interview sessions controls for the participants having bad days. Seidman also stressed that the interviewer should not talk more than necessary to let the participants' words stand alone. I followed all of these recommendations in this study.

Despite the various ways researchers can try to ensure validity, there is always the chance that participants will not share the information the researcher is seeking. According to Barriball and While (1994), it is especially difficult to establish credibility, which requires reliability and validity, in semi-structured interviews. They stated that semi-structured interviews "are well suited for the exploration of the perceptions and opinions of respondents regarding complex and sometimes sensitive issues and enable probing for more information and clarification of answers" (p. 330). In other words, semi-structured interviews keep enough standardization for researchers to compare 
different respondents, but allow for flexibility so that researchers can clarify and explore more deeply with each respondent. In interviews, it is important to watch out for answers that reflect expected social responses rather than the truths of each respondent. Barriball and While pointed out that significant differences between the researcher and respondent can result in an increase in answers reflecting expected responses rather than personal beliefs.

I used semi-structured interviews (Barriball \& While, 1994) to ensure understanding among participants who are not native English speakers, and to avoid problems common in more structured interview approaches (Denzin, 1989). I remained flexible in conducting interviews, which ensured mutual understanding so I could gather the data necessary to answer my research questions. If I had used a highly-structured interview protocol, I would have run the risk of participants having different understandings of the questions, and would, therefore, have gathered inconsistent data.

Denzin (1989) brought up the issues of external and internal validity in observational research in asking if the observations can be generalized (external validity) and if the observations represent real differences (internal validity). According to Cronbach (1975), observational research can be generalizable after many detailed descriptions of particular situations are taken together. Denzin (1989) argued that observational research could be generalizable if the cases studies are representative of the larger population. For the purposes of my study, I did not seek to generalize outside of the participants in the study, so external validity is not an issue. 
To ensure internal validity, Denzin (1989) argued that the researcher must consider historical factors, subject maturation, subject bias, subject mortality, reactive effects of the observer, changes in the observer, and peculiar aspects of the situations where the observations occur. I focused on the current experiences of the participants in my study, but I took into account historical factors through their interviews and stimulated recall sessions. Because I was studying the experiences of Burmese refugee students in middle school, their maturation and bias were part of the data I gathered and, therefore, were not a threat to validity. To address reactive effects and changes in myself as the researcher, I was sure to check any of my hypotheses from observation against data I gathered in interviews and stimulated recall. Finally, I addressed peculiar aspects of the situations where observations occur by fully describing the situations and allowing for participants to describe their experiences in those situations in interviews and stimulated recall sessions.

Another way I checked the validity of the data was through member checking of the interview transcripts. Member checking gave the participants a chance to read transcripts of their own words so they could verify or clarify anything they meant to say. A final strategy described by both Creswell (2013) and Maxwell (2013) was providing a rich description of the experiences of Burmese refugee students in multicultural middle school classrooms. To Creswell (2013), this meant describing in detail, including contexts of different situations and the actual words of the participants, the phenomenon I describe. I provide rich descriptions in Chapter 4. 
Together, long-term engagement, familiarity with the community, triangulation of the data, member checking, and rich descriptions do not necessarily ensure validity. The evidence I gathered from using these strategies, however, increases the likelihood that the data are valid.

\section{Role of the Researcher}

This study was difficult from a positionality standpoint because I had been the teacher of each of the participants. At the time of the study, I was the social studies teacher for two of the three participants. I was the social studies teacher for the other participant the year before the study began. Seidman (2013) explicitly warned against teachers interviewing their own students due to the power differential.

To mitigate any undue influence, I was careful to not coerce students into participating or unintentionally influence their responses. Following the advice of Denzin (1989) to make the researcher's role clear and present himself consistently, I made clear that my roles as teacher and researcher were separate; I explicitly stated how students' grades and treatment in class were not affected by their participation or nonparticipation in my study, and their comments during the course of the study would in no way affect my treatment of them as students. The primary way I addressed my dual role was to conduct the research outside of my own classroom. Though I taught two of the participants during the 2014-2015 school year when the study took place, I gathered all data outside my role as a teacher. I conducted interview and stimulated recall sessions at the participants' convenience during their lunch or after school. I conducted observation 
sessions in other teachers' classrooms during my preparation periods when I was not teaching.

In addition to trying to separate my roles as teacher and researcher explicitly, I addressed my positionality in several other ways. I gathered data using a variety of methods (i.e., interview, observation, stimulated recall). Using multiple methods increased data reliability (Krathwohl, 2009) and validity (Creswell, 2013; Maxwell, 2013). I had a long-term investment with these students during which I built rapport (Creswell, 2013; Maxwell, 2013). I have been teaching and working closely with Burmese refugee students for three years, and I have built relationships with many students and their families. I have worked to help them understand that while I have been interested in their experiences in the United States, I was also their teacher and treated them fairly in class - by not giving them special treatment in any way, nor by punishing them for not participating. I remained fully aware of the dual relationship in which I inhabited during the study and was as open as possible with the students and families to explain my roles.

According to Vogt et al. (2012), each research design comes with a variety of ethical issues, specifically in the areas of consent, harm, and privacy. In all parts of my study I had to be careful with the children's consent as they were vulnerable and from marginalized populations, be aware of my positionality as a teacher to avoid coercion, and be sure to report only relevant information to protect everyone's privacy. I had to be especially aware of my participants' positions as both students and refugees. Especially when I interviewed them, I had to be careful to not trigger any painful memories or give 
them the impression that they were being forced to participate in any way. This was particularly difficult as I was not aware of the specifics of my students' upbringings. I

did my best to pay careful attention to their answers in the interview portion of the study. Because I used a semi-structured interview design, I was able to be responsive to any potential incidents of students' resistance. This did not happen frequently, but each participant had some moments in which they were either unwilling or unable to answer questions. In those cases, I tried to steer the interview toward a more effective topic.

Because I was a full time teacher while researching the experiences of Burmese refugee students in their classrooms, time considerations were especially important. I was unable to conduct interviews or stimulated recall sessions during most of the school day. I could only conduct observations during my preparation period when I was not teaching. I ended up scheduling interview and stimulated recall sessions during lunch and after school at the convenience of the participants.

\section{Data Collection and Analysis}

I collected data during the spring of the 2014-2015 school year, using the aforementioned methods. I conducted interviews over the span of four weeks. I audiotaped and took notes during each interview. After I transcribed the full interviews, I gave the transcript to the participants to member check. I conducted observations over the span of three weeks after finishing the interviews. I took field notes and videotaped each formal observation. Then, I used the video from the observations for the stimulated recall sessions, which I conducted over the span of two weeks. During the stimulated recall sessions, I played the video of the observations on my computer for the participant 
to view and used a screen capture function to audio record our comments synced with the video playback on my computer screen. This allowed me to easily access the data I collected from all three methods through interview transcripts with interview notes, video recorded observations with field notes, and audio recordings of stimulated recall sessions synced with video screen shots of what we watched on the computer during the session. I finished collecting data approximately one week prior to the end of the school year.

For analysis, I followed a general process as described by Maxwell (2013). I began by reading the interviews and assigning tentative codes. Creswell (2013) called this step finding, "significant statements" (p. 82). I assigned codes within each interview and across the interviews with all three participants. Maxwell (2013) called the process of identifying codes based on the words of the participants, "descriptive" (p. 108). The intent of the codes was to accurately capture the experiences of the participants. I selected codes in light of Bourdieu's theory of field (1977) and theory of cultural and social reproduction (1973). I also paid attention to how a transcendental phenomenological study is analyzed according to Moustakas (1994), in which the researcher attempts to explain the essence of the experiences of the participants by bridging their noema, or experience of a phenomenon, and noesis, or interpretation of their experiences.

Throughout the study, I continually reassessed previously-gathered data in light of new information to generate preliminary ideas of possible themes. When I finished gathering the data, I checked the tentative themes I identified from the interviews against the data gathered from the observations and the comments participants made during 
stimulated recall sessions to help shape the final themes. Once I identified themes based on the interviews, I again checked them against the data I gathered through observation and stimulated recall.

In the end, I identified nine themes (i.e., dichotomy of experiences, importance of Islam, importance of family, importance of friends, importance of being nice, struggle in class, importance of learning, importance of speaking English, and importance of being on task), which I regrouped into three categories (i.e., focus on understanding what they were learning, the importance of staying on task, and the importance of being kind to others) described in Chapter 4 (see Table 3). While identifying the themes, I was careful to link them to my three research questions:

1. What were the experiences of Burmese refugees in multicultural middle school classrooms?

2. How did Burmese refugee students experience interactions with students from other cultural backgrounds?

3. How did the interactions Burmese refugee students have with students from other cultural backgrounds affect their school experiences, including learning and identity formation?

The data gathered from interviews, supplemented by data from stimulated recall, primarily informed the first research question. I addressed the second research question using primarily stimulated recall, supplemented by observation and interview data. For the third research question, I used data gathered from all three methods. 
In light of Bourdieu's theory of field (1977) and theory of cultural and social reproduction (1973), the first research question addressed the dispositions of the participants and how they interpret the necessary habitus to be successful in the field. The second research question addressed the habitus the participants thought was necessary to be successful and how the students worked to internalize that habitus. It also addressed whether the participants thought they were working toward gaining the cultural capital necessary to be successful. The third research question addressed the participants' perceptions of how the classroom environment and opportunities for intercultural interactions affected their work toward gaining cultural capital.

In light of the process for a transcendental phenomenology (Moustakas, 1994), the first and second research questions mostly addressed the participants' noema, or experiences of the phenomenon. The third research question more directly addressed the participants' noesis, or interpretations of their experiences. Of course, the data I gathered from all three methods for the three research questions overlapped.

In Chapter 4, I use the collected and analyzed data to describe the experiences of Burmese refugee students in rich detail, as advocated by Creswell (2013) and Maxwell (2013).

\section{Conclusion}

To study the experiences and intercultural interactions of Burmese refugee students in a multicultural middle school, I used a qualitative (Creswell, 2008; Krathwohl, 2009), transcendental phenomenological approach (Moustakas, 1994). First, I interviewed the students to understand their perspectives on school in the United States, 
which primarily addressed my first research question. Then, I observed them interacting with students from different cultural backgrounds. Those observations were videotaped. After the observations, I conducted stimulated recall sessions with the participants to gain insights into their experiences during intercultural interactions.

I used purposeful sampling (Maxwell, 2013) to select three female Burmese refugee students enrolled in seventh and eighth grade to participate in my study at the school where I teach. Two of the students identified as Rohingya; one identified as Burmese. All three identified as Muslim. All three participants ranged from ELL level three to five (monitored). I clearly explained to the participants my role as a researcher. I knew them well as their teacher, but their participation did not affect their grades or treatment in class. To avoid potential power differentials, I conducted the research outside my classroom. I was also sensitive to their unique needs as refugees.

I analyzed the data by coding the interviews in light of Bourdieu's $(1973,1977)$ theories. I also kept in mind the process of transcendental phenomenological analysis (Moustakas, 1994) in which the researcher seeks participants' experiences and interpretations of those experiences. Because my study was iterative, I continually reassessed previously-gathered data in light of more recent data.

Using these three methods, and bracketing out my experiences, I developed a picture of the experiences the participants have in the multicultural middle school where I teach. Because so little is understood about Burmese refugee students' experiences in classrooms, my study will help other researchers understand one further piece of how different refugee groups fare in their new countries. In the next chapter, I describe my 
analysis of the data and what I discovered about how female Burmese refugee students experience multicultural middle school classrooms. 


\section{Chapter 4: Results and Analysis}

In the previous three chapters, I explained how Burmese refugee students who attend the multicultural middle school where I teach do not interact with a wide variety of students from other cultures despite the benefits that intercultural interactions may have for marginalized students, detailed the research related to the problem of practice, and outlined the methods I employed to answer my research questions. The purpose of this study was to examine the experiences of three female Burmese refugee students in their multicultural middle school, especially focusing on their intercultural interactions, to better understand why they were not interacting with students from a variety of backgrounds.

In Chapter 1, I argued that refugee students in multicultural classrooms have many opportunities to interact with a range of students, which could help them overcome their challenges (McBrien, 2005) and gain access to the benefits of the hegemonic culture (Phinney et al., 2007; Ryan \& Patrick, 2001; Wang \& Eccles, 2012; Wenzel et al., 2012). To try to understand why Burmese refugee students were not interacting with a wide range of students, I proposed a study framed around three research questions:

1. What were the experiences of Burmese refugees in multicultural middle school classrooms?

2. How did Burmese refugee students experience interactions with students from other cultural backgrounds? 
3. How did the interactions Burmese refugee students have with students from other cultural backgrounds affect their school experiences, including learning and identity formation?

In Chapter 2, I explained that I approached this study through the lens of Bourdieu's theory of field and theory of social and cultural reproduction (Bourdieu, 1973, 1977, 1990; Bourdieu \& Wacquant, 1992), in which people bring their dispositions into a field, which develops successful habitus. Those whose dispositions most closely match the habitus of the field gain more capital and, thus, help to shape the habitus. People whose dispositions do not match the habitus in a field have less capital and are marginalized.

Also in Chapter 2, I reviewed the relevant literature stating that middle school is a time when young adolescents shift their focus from their parents to their peers while forming their identities (Erikson, 1950, 1968; Larson \& Richards, 1991; Smetana et al., 2006). Minority students especially benefit from interacting with peers in multicultural contexts in forming their ethnic identities (French et al., 2006; Marcia, 1966; Phinney, 1989). The school where I conducted the study was what Vertovec (2007) called superdiverse. In such a multicultural context, it is incumbent upon educators to provide, “equitable learning opportunities" (Lee, 2003, p. 466) for students to maximize their learning. Multicultural researchers argue that schools need to foreground students' backgrounds so that they feel empowered (Banks, 1993; Bennett, 2001; Daoud, 2003; Gay, 2004; Malsbary, 2014; Martinez, 2010; Paris, 2009; Sleeter \& Grant, 1987). In multicultural contexts, students have ample opportunities to interact with students from a 
variety of different cultural backgrounds. Intercultural interaction can benefit students in many ways, including reducing prejudice, forming one's ethnic identity, learning the norms of the dominant society, and navigating the school system (Allport, 1954; Gurin et al., 2011; Pettigrew \& Tropp, 2006, 2008; Ryan \& Patrick, 2001; Wentzel et al., 2012; Yoon, 2012). The benefits that come from intercultural interaction can especially help refugee students, who have particular challenges that make adjustment to their new homes difficult (McBrien, 2005; Valenta, 2010). A variety of supports exist, however, that can help refugee students with their transitions, including community-based, schoolwide, and classroom-based programs (Banki, 2012; Block et al., 2014; Dávila, 2012; Dooley, 2012; Ferfolja \& Vickers, 2010; Kirova, 2012; McBrien \& Ford, 2012; Pugh et al., 2012; Taylor \& Sidhu, 2012). Female refugee students, in particular, seem to bridge their experiences before resettlement with looking toward the future to become successful (Harris, 2010; Oikonomidoy, 2007, 2009). My study focused on refugees from Burma, also known as Myanmar, who constitute one of the largest incoming refugee groups in recent years (Martin \& Yankay, 2012; UNHCR, 2015). Despite their relatively large numbers, their experiences in schools in the United States have been lightly studied (IsikErcan, 2012). My study fills a gap to illuminate the experiences of three Burmese refugee students in my multicultural middle school.

In addition, I outlined the literature behind my methodological approach to the study. I used a qualitative (Creswell, 2013), transcendental phenomenological (Creswell, 2013; Moustakas, 1994) approach in which I interviewed the participants, observed them 
in a classroom, and conducted stimulated recall sessions using video from the observations.

In Chapter 3, I detailed how I conducted the study. I used a qualitative design to explore one topic in depth (Creswell, 2013; Krathwohl, 2009). My design came from a constructivist paradigm (Guba \& Lincoln, 2005), in which reality is subjective and researchers seek the participants' understanding of the world. More specifically, I took a transcendental phenomenological approach (Moustakas, 1994), in which researchers seek to fully understand and report the experiences of a few participants.

I chose my participants based on convenience, or purposeful, sampling (Creswell, 2013; Maxwell, 2013). The participants were all students who were in classes during my preparation period (when I was not teaching) in which they interacted with students from other cultural backgrounds. All three were females in seventh or eighth grade who identified as Muslim and were in ELD levels three, four, or five. Two participants identified as Rohingya, while one identified as Burmese.

To answer my three research questions (e.g. What were the experiences of Burmese refugees in multicultural middle school classrooms?, How did Burmese refugee students experience interactions with students from other cultural backgrounds?, and How did the interactions Burmese refugee students have with students from other cultural backgrounds affect their school experiences, including learning and identity formation?), I gathered data from interviews, observation, and stimulated recall.

I conducted semi-structured interviews (Barriball \& While, 1994; Seidman, 2013) to gain an initial understanding of the experiences and perspectives of the participants. 
After the interview portion, I conducted observation sessions (Bailey, 1994; Vogt et al., 2012) to watch the participants interact with students from other cultural backgrounds. I video recorded each observation for use in the stimulated recall sessions. After the observations, I showed the participants video of themselves and asked them to comment upon parts they thought were important. During the stimulated recall sessions (Bloom, 1953), I took video screen shots of the observation videos with audio recordings of the participants' comments overlaying the video.

I analyzed the data by coding the interviews in light of Bourdieu's $(1973,1977)$ theories. During analysis, I also paid attention to the participants' experiences and interpretations of those experiences as called for by a transcendental phenomenological analysis (Moustakas, 1994). As I gathered new data, I continually reassessed previously gathered data to arrive at my final themes. In this chapter, I detail how I analyzed the data and report the results.

\section{Analysis of Data}

To analyze the data, I generally followed a coding process described by Maxwell (2013) and a transcendental phenomenological analysis process described by Moustakas (1994) and Creswell (2013). First, I read the interview transcripts to identify tentative themes both within each interview and across the three interviews (see Appendix C). I used the tentative themes to guide my observations, while also comparing my observations to the interview data to check that I had not missed any possible themes. As a subsequent validity check, participants commented on their filmed interactions with other students. At this stage, I again checked my tentative codes against the new data. 
When I finished gathering data, I finalized the descriptive codes (see Appendix C) based on the interviews that were supported by data from observations and stimulated recall. Then, I collapsed the nine initial themes (i.e., dichotomy of experiences, importance of Islam, importance of family, importance of friends, importance of being nice, struggle in class, importance of learning, importance of speaking English, and importance of being on task) into three larger categories (i.e., focus on understanding what they were learning, the importance of staying on task, and the importance of being kind to others) that provide a picture of the experiences female Burmese refugee students have in multicultural middle school classrooms (see Table 3).

\section{Presentation of Results}

In this section, I identify the themes, explain how the participants discussed the themes, and use those themes to address each research question. For each research question, I describe the participants' experiences (noema) and their interpretations of those experiences (noesis), as called for in a transcendental phenomenological study (Moustakas, 1994).

In the interviews, I identified nine initial themes consistent across the three participants. One theme, the theme of dichotomy of experiences, ran throughout the study, as participants described various phenomena in an either/or manner. The two most obvious examples came in how participants described their experiences both prior to entering the United States and after they arrived, and in describing students as either good or bad. In addressing the research questions, I did not bracket this theme out because it seemed to be an underlying philosophy of the participants and manner in which they 
discussed phenomena, rather than something they outwardly talked about like the other themes I identified.

I combined the other eight initial themes (i.e., importance of Islam, importance of family, importance of friends, importance of being nice, struggle in class, importance of learning, importance of speaking English, and importance of being on task) into three large categories: (a) focus on understanding what they were learning, (b) the importance of staying on task, and (c) the importance of being kind to others (see Table 3). I included the importance of Islam into the category of staying on task because the participants discussed their experiences with their religion that emphasized following the directions of an adult.

Table 3

Initial Themes Aligned to Categories

Initial Themes

Dichotomy of experiences

Struggle in class Importance of learning Importance of speaking English

\section{Categories}

All three categories

Focus on understanding what participants were learning
Importance of Islam

Importance of being on task
Importance of staying on task

$$
\text { Importance of being kind to others }
$$

Importance of family Importance of friends Importance of being nice 
The three categories, focusing on understanding, staying on task, and being kind to others, combined to create a model for the students of what a good student should be.

For the first research question (What were the experiences of Burmese refugees in multicultural middle school classrooms?), which I primarily addressed via interviews, the participants discussed focusing on understanding, staying on task, and being kind to others. For the second research question (How did Burmese refugee students experience interactions with students from other cultural backgrounds?), which I primarily addressed via observation and stimulated recall, the participants appeared to work equally well with students across ethnic backgrounds. They reported in stimulated recall sessions they acted like their ideas of good students, and would do so regardless of their partner's ethnic background. For the third research question (How did the interactions Burmese refugee students have with students from other cultural backgrounds affect their school experiences, including learning and identity formation?), which I addressed via a combination of all three methods, students showed they fulfilled their ideas of being good students by focusing on understanding, staying on task, and being kind to others. They seemed to take some of those ideas from their memories of the countries from which they moved (Thailand, Bangladesh, and Malaysia).

Next, I detail the results, framing them around the three themes I identified (focus on understanding, staying on task, and being kind to others). For each theme, I describe the participants' neoma, or experiences (Moustakas, 1994), using direct quotes by the participants as much as possible. I chose to represent their patterns of speaking as closely as I can, which means leaving in any grammatical errors or misuses of words. I discuss 
BURMESE REFUGEE STUDENT INTERACTION

the experiences of each participant separately within each of the themes. Then, I connect my conclusions to the research questions.

\section{Focusing on Understanding}

The first major theme I identified with the participants was a focus on understanding the material they were supposed to learn. For all three participants, it was important to understand what they were doing, not simply do the work to get it finished. Understanding, though, was a struggle for all three, especially when it came to learning English. All three participants seemed aware that they were able to reach understanding of the material even while they struggled to grasp certain things. Learning English was a key part of the process of understanding for all three participants.

Ohma. Ohma did not say very much during interviews about how important she thought it was to seek understanding, but she expressed two powerful statements that spoke to how crucial it was for her to understand what she was learning. In both quotes, she made clear the connection between struggling and understanding. In the first, she said:

Sometimes, like, I don't really get how they [the teachers] say it, so, like, I have to ask the teacher and then, like he will say, like, really simple, and then I'll like, read the sentence again it was, like, so difficult, so like, I listen to the teacher instead of, like the sentence on the paper.

In other words, when she did not understand what she was reading, even after the teacher tried to explain it to her, she would keep trying by listening to the teacher's explanation 
again. She later acknowledged the process of understanding what she was reading was becoming easier as she was acquiring more and more English.

In a second quote, Ohma framed the importance of understanding around helping other students understand, which, in turn, gave her a better grasp of the concept. When talking about working with a partner chosen by the teacher, she stated, "I try my best to, like, make them understand stuff, and like, I understand stuff. So, like, if... but, like... if that doesn't happen, I try to ask the teacher."

To Ohma, working with other students was beneficial even when it was someone helping her. She stated, "If I don't know something, then they'll help me, like, figure it out." She trusted that, even when she did not understand a concept, someone (either the teacher or another student) would help her and she would eventually grasp it.

What Ohma said during interviews was borne out in observations. Ohma was in a loud science class with many transitions. During one observation, the teacher gave a long (approximately ten minute) introduction to the lesson, in which students were asked to identify objects hidden in sealed film containers. Once the activity began, students worked with a partner shaking the containers, writing down their guesses, discussing their guesses with partners, asking the teacher for help, and trading in their containers for new ones. Because of the activity, the class felt very energetic. There were many opportunities to refuse to participate or simply copy from other students. Ohma, however, sought out understanding rather than resorting to copying from other students. Several times, she collaborated with her partner, a female student who identified as Turkish and Muslim, to arrive at hypotheses. On one occasion, Ohma asked the teacher 
for help when neither she nor her partner had a guess about what the container held. The teacher responded that she should test her hypothesis that the container was empty.

Ohma proceeded to shake an empty container and seemed satisfied that the test container was empty.

In a stimulated recall session, Ohma reported asking her teacher for help because she was confused about not being able to hear any sound. In her words, she, "had to ask." When I asked her why she asked her teacher instead of her partner, she answered that she had already asked her partner, who also did not know. She resolved her confusion by shaking a container she knew was empty and comparing it to the test container.

In another part of the activity, while her assigned partner was getting another container, Ohma tossed a canister to another student (a Caucasian American female) and asked her for help with identifying the contents. The other student did not know and tossed the container back to her. Ohma continued shaking it and testing various materials in the hopes of finding out what was in the canisters.

Even when working with a friend and given an opportunity to copy something to just finish an assignment for a grade, Ohma preferred to work toward understanding. In one observation, Ohma asked a friend of hers from Nepal for help on an assignment about the periodic table. The friend came to her, explained what she was supposed to do, and Ohma began working on the assignment. When telling me about this interaction, she said, “I just don't copy. Then, I really don't know what I'm doing and then I get 
confused easily." Clearly, understanding what she did was more important to her than getting a good grade on the assignment.

How well she and others spoke English was something Ohma referenced often, perhaps because she was learning the language herself. Sometimes, she referenced how others' English language ability impacted her understanding of what she was supposed to learn. In discussing working with other students, Ohma said a student's cultural background only matters to her when it comes to how well he or she speaks English. The ethnic backgrounds of students otherwise did not matter to her. When she discussed what it would be like to work with another Burmese student, she said it would be difficult because that student would not understand enough English to do the assignment on her own, so she would ask Ohma too many questions for her to get her own work finished. Even though she said in her interviews that helping others could increase her understanding of the material, when it came to students who did not speak English well, she thought they impeded her learning. Interestingly, she identified only Burmese students as those who did not speak English well. As such, Ohma preferred to work with students from other cultural backgrounds so that she could focus on her own understanding of the material.

In sum, Ohma strongly sought out understanding the material, even when she struggled to understand or had the opportunity to copy information to complete an assignment and, presumably, earn a good grade without much intellectual work. In fact, she directly acknowledged how struggling leads toward understanding and how copying impedes understanding. 
Khalidah. Like Ohma, Khalidah acknowledged how struggle leads toward understanding, especially when it came to learning English. Though Khalidah had acquired a more complete understanding of English than Ohma, she still spoke of her experiences acquiring the language.

Khalidah said that reading was the most difficult thing to learn when she moved to the United States, which hindered her understanding in school. When I asked her what was so difficult, she answered, "They [the letters] were difficult. I didn't see those kind of letters before." This makes sense, as her native language was Bengali, a language with very different looking letters than English.

Her struggles in learning English seemed to lead Khalidah to a clear understanding of how to ensure she learned as much as possible. She reported wanting to work with other students so that she could learn more when she said, "The more I work with people, the more I get in my head. And that helps me think more. And, ya, that way I get my tasks done faster." She later restated the same idea by saying, When people talk or things... it comes... It kind of gets me to think more. I don't know why how that works, but I just think more when peoples like working it together, instead of, like, individually. Because when you need help, the teacher's with another person, you know?

It was interesting in this quote that she showed a preference for asking a teacher for help, but understood that teachers were often busy, so asking a peer was necessary. 
Even though Khalidah valued working with others, she also took pride in her individual accomplishments. She was especially proud of her progress in reading. Speaking about her reading support class (Read 180), she shared,

Last year, in seventh grade, I won a, um, student of the month for, like, finishing a whole... mostly the whole thing. Like, six more levels until I finish the whole, um, Read 180 thing. That website. Ya. Like six more, and I was going to be finished.

Khalidah generally did not ask for help, taking the lead with partners during most observation sessions. However, during one session in which the class was supposed to fill out a worksheet explaining various causes and effects of the Civil War, she and her partner called the teacher over to help them. Both students had most of the work already finished, but needed guidance to finish. Once the teacher guided them, they worked well together to finish the worksheet.

I did not see Khalidah ask for help in any other observation session. None of the work appeared to be particularly difficult, which Khalidah confirmed during stimulated recall session. Part of the reason she consistently took the lead with partners was she believed she had a better grasp of the material and was usually farther along on assignments than her partners.

Overall, Khalidah knew she had previously struggled to learn English, and, thus, worked very hard to understand the material in her social studies class. As such, she was typically more focused on her work than her peers and was in a position of helping them when she was partnered with others. 
Shamshidah. Both Ohma and Khalidah spoke clearly about how their struggles in school, especially in acquiring English, led to greater understanding of the material. Shamshidah, partially because of her language level, struggled with academics more than the other participants. Speaking of difficulties in school generally, Shamshidah said, "Some studies hard. Um, we have a lot of tests. We get to write a lot. That's all." She also said of her classes, "Because they're hard. Some confusing too." Shamshidah stated that reading was difficult for her, but was not able to give any more details. When I asked her why she thought reading was so difficult, she said, "But I just doesn't like it." I attribute her inability to clarify to her English language acquisition level, which was lower than the other participants.

Despite her struggles, she still wanted to learn and do well academically, as evidenced by her earlier quote, "Because, because it's how I can be... it's how I can take my careers. So it opens my knowledges." She also said of her favorite classes, "You get to study a lot. You get to write a lot." I understood this, based on her body language, as a positive statement; she thought studying and writing were important and took pride in doing those things. Later, she stated, "I like to study a lot." When I asked her why she enjoyed working in class, she answered, "So I can think well."

Though she did not clearly explain how struggle leads to understanding, Shamshidah clearly understood that struggle was not an impediment to understanding, but a step in the process. She wanted to do well, knew she could do well, and knew that things were difficult for her at the present time. 
Acting out her acknowledgement of her struggles in school, Shamshidah frequently asked her partner for help during all of the observation sessions. I observed her in a seventh grade math class during the last period of the day. During each observation, the teacher asked students to complete a series of answers, then discuss the either the answer or process they used with a partner. Partners were seated at tables together, so Shamshidah's partner was the same Caucasian American female throughout the observations.

Almost every student in the class participated, though to varying degrees. For a class in which students were supposed to be discussing answers, it tended to be very quiet. In every occasion, Shamshidah could not finish the math problems during the time allotted, and her partner guided her toward the answers. During two of the observation sessions, she asked her partner for help prior to starting the problems. Her focus seemed to be on understanding the correct process before trying to discuss her answer with her partner.

During a different observation session, the teacher asked students to solve multiplication problems with decimals, then explain their process to their partner. Before starting the work, Shamshidah turned to ask her partner a question. After solving the first problem, she actively engaged her partner and fully explained her process. The partner helped her find two mistakes, which she quickly fixed.

Shamshidah spoke about seeking understanding during stimulate recall. In one session, she said even though the directions were to share her answer, she thought it was important to try to finish the problem first. She wanted to share her answer, but she had 
to first understand what she was doing. While watching a different video clip, Shamshidah shared that sometimes it takes her longer to finish problems, so she prefers to wait to share with her partner until she finishes the problem. This was my working assumption during the observations, and Shamshidah confirmed she would not share until she knew exactly how to do the problem. Understanding what she was doing was more important to her than simply completing the task.

Later in stimulated recall, she watched a video of her asking a question to her partner about the directions. In the video clip, however, it was difficult to hear exactly what was said. Shamshidah explained that she asked her partner to clarify directions, which her partner did. In the clip, Shamshidah began writing as soon as her partner answered her question. When I asked if her asking for help was a normal occurrence, she said it was in math, but students in other classes would sometimes ask her for help. Supporting her assertion, I observed Shamshidah ask her partner for clarification before starting any work on two separate occasions before I began filming.

In sum, Shamshidah struggled with understanding the material more than the other participants, but she worked very hard to do so. When she did not understand, she was willing to ask her partner for help.

Summary. Despite struggles in learning English, Ohma, Khalidah, and Shamshidah worked very hard to understand the material they were supposed to learn. They were consistent in their statements and actions, showing their commitment to seeking understanding. They seemed to understand very well that the purpose of school 
was to learn, and finishing assignments was simply a way to ensure learning. As such, they focused on the learning rather than just going through the motions of finishing work.

For each of the participants, a large part of ensuring they understood the material was making sure they were following directions and staying on task. The second major theme I identified was that the participants put a high importance on staying on task in their classes.

\section{Staying on Task}

One way the participants ensured that they were learning the material they were supposed to learn was to focus on staying on task in the classroom. Though staying on task most likely helped them understand what they were supposed to learn, they all spoke of the concept independent of seeking understanding in their classes. Staying on task was a way to do well in school, but according to the participants, it seemed to have value in and of itself.

All three participants were Muslim and had attended Islamic schools at some point. When they discussed their religion, they framed their comments in terms of being compliant and following directions. For this reason, I included their emphasis on religion as a part of the staying on task category.

Staying on task was especially important because the participants believed many other students did not try to do well in school, which annoyed them. They seemed to group students into those who did well and those who did not. Similarly, they dichotomized their experiences in their countries of origin with their experiences in the United States. Despite the differences between their experiences, they seemed to use the 
values they learned in their countries of origin to inform their habits in schools in the United States. Some of the values they used to inform their habitus came from their experiences of being compliant in Muslim schools.

Ohma. Ohma clearly reported the connection between staying on task and doing well in school when she said, "When we talk too much, we don't, like, finish our work. And, like, if we don't finish our work, we will have bad grades and ya." Getting good grades was obviously important to Ohma, and being compliant in class was a major factor for her.

When other students were off task, she reported growing annoyed by them and wanting to let the teacher know, but not wanting to disrupt class in doing so. Ohma seemed to group students who did not do well together as those who copied off others. She stated about working with off task students, "If they just want to copy off, I will... I will, like get mad, but like, I will be staying still and, like, saying nothing. So, like, I don't really express the madness." When I asked her how she might let the teacher know when she was annoyed about copying, she said, "I will, like, say another state that doesn't recommend that copying part. Like, I will say something else. I will say something else that bothers me more than the copying part." Though her exact meaning is somewhat unclear, I believe she meant that she would not tell on another student directly, but she would tell the teacher something else that might push the teacher to watch the copying student more closely. She would not let herself get distracted by the off-task students so that she could stay on task. 
Ohma seemed to see students as either good or bad. Similarly, she saw her Muslim school experiences in Thailand as starkly different than those in the United States. Of the room configurations, she said, "It's not like these tables, it's like on the floor tables." When she talked about a teacher in Thailand, she said, "He sit in the front, like right in the front of the class. He don't really use textbook, like yours. He just have this, like, little book about the Koran, and there's, like, so many different kind of books." When describing the classroom in general, she stated, Um, like, it's really big. It's like old, but, like, not like this. We don't have a whiteboard. It's just like wood, and, like... and, like the bathroom is, like, right next to, like, outside of the school. And... there's, um, there's, uh, there's, uh... like a food court outside of the school.

She further stated:

We don't use technology, like right here. And we don't have, like many posters. No crayons, no coloring. And... we don't use... like we don't have, like... um, we actually have outfits, uh, uniforms. And we have to wear scarves, and, like, the teacher, like, has this big stick. If, like we don't get it, he will, like, just hit us on the hand, like [demonstrated hitting on the hand] slowly. But, like, some kids get hurt, but not much.

The punishment for not doing well was one of two instances when Ohma mentioned ways the teacher kept the students on task. When I asked Ohma if she found the differences surprising when she arrived in the United States, she replied: 
Um, yes, because, like, we used to use, like, these technology, and like, there, we just had to sit down, read our stuff, and, like, work as hard as you can. If you don't get it, you have to do these, like, difficult sit and down things.

She went on to act out and describe squats with her arms held out in front of her. These quotes were the clearest Ohma was about having to be compliant in schools in Thailand. To be successful in her previous school, she had to do what the teacher asked and work hard. That was the same attitude she brought to her classroom experiences in the United States.

In observations, Ohma displayed her value of staying on task. She worked with a variety of students from other cultural backgrounds during observations; she always worked constructively and I never saw her off task. Twice, I saw her work in partners; one time she worked with a student who identified as Turkish and Muslim, another time she worked with a friend of hers who was Nepali. In both interactions, she worked diligently.

In the interaction with the student who identified as Turkish, Ohma actively worked to discover the contents of various film canisters during a science lesson. The two spoke approximately the same amount, though Ohma was clearly more animated and had a slightly annoyed tone the whole time due to not knowing the contents of each canister. Belying her apparent annoyance, though, was a constant smirk on her face indicating some acting due to my presence. Despite her apparent annoyance, she never got distracted while investigating the contents of the film canisters. Once she and her 
partner finished the first part of the activity, they dutifully wrote what they found on an organizer provided by the teacher.

Later in the same activity, both Ohma and her partner got up to exchange their canister for another one. There was some discussion back and forth, and Ohma went back to her seat. During stimulated recall, Ohma said that the directions were for one partner to stay at the seat at all times. She and her partner informally decided to alternate instead of having one student get the canisters every time. They were fewer than 10 feet away from the canisters, so it is unlikely the teacher would have noticed if they both went up. It was important enough to Ohma and her partner to follow directions, though, that they spent approximately five seconds going back and forth about who should stay and who should go before one of them retrieved the canister.

In the other partner interaction, Ohma asked her friend (a student from Nepal) for help on an assignment about the periodic table. I had seen Ohma be quite silly and play around with this particular student on many occasions outside of class. At the time she came over in the classroom, the teacher was on the opposite side of the class. I expected that they may have used the opportunity to talk about non-school related things or get off task in another way, but both students stayed on task. Neither partner got distracted during this interaction. In addition, Ohma did not copy any answers. Her friend explained what she was supposed to do without giving any answers. Once Ohma was satisfied with knowing what she should do, her friend moved on to another activity. The entire interaction took less than one minute. 
Even though Ohma worked well with some students from other cultural backgrounds, she was cautious in working with those she did not think were good students. Despite her caution, she still stayed on task during an activity in which she was asked to work with a group. As she explained in her interviews, there were a number of students who she did not trust to work well. In the group work session, she and four other students tried to locate barriers hidden under a box by rolling a ball under it and mapping its path. Her group was made up of Ohma and four girls: one identified as Croatian, two as American, and the fourth as African American. It is interesting to note that during a stimulated recall session, she misidentified one of the girls who identified as American. Ohma and the girl who identified as Croatian took the lead and effectively left the other three out of the activity. Though it would have been easy for Ohma to play with the marble, she stayed focused on the task at hand the entire work session.

When I asked Ohma during stimulated recall if the video showed that she valued staying on task, she replied that she did. When I asked if there were people who she might get off task with, she said that a student who did not do their work might get her off task. She also said that working with friends helped keep her on task, even if she laughed at their jokes. Interestingly, she said one of the reasons she highly valued staying on task was that if she starts to get off task, she has a hard time getting back to her work. She compensated for this by focusing on her work. In all of the observation sessions, I did not see her get off task once.

During a stimulated recall session, while watching the activity in which she attempted to discover the contents of closed film containers, she said she was very 
frustrated; she wanted to know what was in them, but was unable to look inside because the teacher had told them not to. In reality, the teacher was not working with her and her partner, and it would have been possible, even easy, to peek without getting caught. She, however, never disobeyed the teacher's directions.

Ohma reported about another instance in which she asked a student for help who was not her assigned partner; when the other student did not know the contents of the film canister, they tested multiple materials before settling on an answer. Again, it would have been easy to simply peek inside the canister, but the teacher had told them not to, so they did not.

In a stimulated recall session where Ohma was discussing another activity, she pointed out, during a section in which she looked bored, that she had already finished a diagram of a box with hidden pegs while the other three students in her group still worked on it. Instead of doing something else, she waited patiently until the other students finished.

In sum, Ohma highly valued staying on task, even when she had opportunities to not do so. In addition, she was able to stay on task even when working with students who she did not trust to work well. She felt especially strong about students who copied, but she was still willing to work with them if the teacher asked her to do so. Some of her belief that students should follow directions seemed to come from her experiences of school in Thailand.

Khalidah. When discussing staying on task, Khalidah focused much more on other students' behaviors than did Ohma. Despite not discussing the inherent individual 
benefits in staying on task, Khalidah took a similar approach as Ohma when annoyed by others. She said of working with students who were not on task, "I get mad, and then I don't like working anymore. So I just sit there quiet while we do the work." She felt fairly powerless in letting the teacher know that other students were off task. She reported her perception of how teachers respond by saying, "They usually say, um, 'just try. Next time I'll try to put you up with another person.' That's what they usually say.' Even though she said she didn't like working when placed with a person by the teacher, when I asked her with whom she preferred to work, she stated, "I'm okay working with anyone." This suggests that she placed high importance on following the teacher's directions even if she did not want to do the exact thing the teacher was asking her to do.

Like Ohma, Khalidah took ownership of her country of origin, referring to Bangladesh as, "my country." She described her Muslim school in Bangladesh by saying:

It's fun. The schools are, like, really different. The schools... there's like only, I think, I don't know, the... Kindergarten, you don’t really study. You play with, like, dolls and things. First grade, I think is [inaudible]. We don't like, really study, but they teach us songs and things, like in our country though. In our own language.

She further described differences between school in Bangladesh and the United States by saying, "Um... the food. It's like, really different. And, I think the time. I think it's like... the time is like, like, five hours maybe. They just give milk and cookies for lunch." I clarified with her that she meant they attended school about five hours per day 
and the only meal they received was milk and cookies at the end of the school day. Khalidah went on to say:

In our country, they usually, the teacher, she works with us, too, like the whole class period. But in here, it's like, they tell you to do your own things and you go to, like different classes. And you change. But in our country, we only have, like, one class. One teacher.

Khalidah could have been describing differences between elementary and middle school, rather than differences between schools in different countries. She seemed to, though, believe there were significant differences between school in Bangladesh and the United States. As one example, she stated, "We worked as a whole class, actually," emphasizing her earlier statement that in the United States, they worked in smaller groups than in Bangladesh. Another time, she said, "The teacher. He's the one that's mostly talking." Though she did not speak to any specific behavioral expectations in Bangladesh, Khalidah's statements that the entire class worked together and were led by the teacher indicate an expectation of conformity and following the rules. Once, she made it clear that they were supposed to follow the rules. She said that if they didn't, the teacher would punish them in much the same way described by Ohma. When describing what would happen if they did not follow the rules, she said, 'Um, if you don't do something they tell you to do, they like hit you with a stick." When I asked her more about her statement about hitting, she made clear they did not hit hard and it did not hurt. She did not make clear what particular behaviors might earn a student getting hit. 
Even though there were times when Khalidah did not want to work with certain other students, it was obvious Khalidah enjoyed school in the United States, and valued staying on task. When she compared school in Bangladesh to school in the United States, one of the main differences she pointed out was that in Bangladesh, they did not get time to work either independently or in groups. She said, "The teacher, she works with us, to, like, the whole class period. But in here, it's like, they tell you to do your own things and you go to, like different classes." Working without direct guidance from the teacher requires students to stay on task to learn, which Khalidah previously stated was important to her. During observation sessions, Khalidah seemed to act out her belief that staying on task was important.

Khalidah worked with four different students while I observed her. All of the interactions were with one student at a time. Three were female and one was male. The male identified as Hispanic, as did one of the females. One of the females identified as Caucasian, the other as Nepali. Khalidah worked equally well with all of the partners, ensuring they finished the various tasks.

Khalidah, aside from bouncing an eraser one time after finishing her assigned task, was on task during every observation session. In one observation, she worked with two separate partners, one at a time. The two partners were both from Nepal and relatively quiet. Khalidah had previously identified each of these females as friends of hers. Their directions were to identify main events from chapter four of the novel Nightjohn by Gary Paulson. Khalidah and her first partner worked well together, though Khalidah did the majority of the talking. When working with her second partner, neither 
Khalidah nor her partner talked, but both accurately completed the section of the worksheet assigned to them.

In another observation session, Khalidah worked with three different partners to identify important events leading up to the United States Civil War. With each of the three partners, Khalidah either led the discussions or was ahead of her partner on the assignment. As such, she often explained the answers to the others. First, she partnered with a Caucasian female who was fairly outgoing and hard working. They collaborated well together, easily finishing their section within the allotted time. With her second partner, a Hispanic male who did most of the talking, but had a lot of work to complete, Khalidah continued to stay on task even while her partner wrote his answer. Her partner became distracted toward the end of the brief (almost three minute long) work time and began talking with other students, but Khalidah worked ahead on the assignment instead of letting herself get distracted. The third partner was a relatively quiet Hispanic female who generally stayed on task quite well. With very little talking, the two partners finished their task in less than two minutes. When they finished, Khalidah bounced an eraser off the floor, marking the only moment I observed her off task. The work time ended within one minute after she bounced the eraser.

Like Ohma, Khalidah talked about not trusting other students to do their work. Her mistrust of other students to work could be a reason she took the lead to make sure she got her work done. Even with two friends (the Nepali girl and the Hispanic girl), Khalidah did not allow herself to get off task until she finished the work. She had many opportunities to get off task, but chose to focus on doing the assigned activity instead. 
Khalidah shared during a stimulated recall session that she listened carefully to directions so she always knew what to do. When I asked her what she was supposed to be doing during one video clip, she was able to tell me the directions for every part of the activity even though the actual activity had happened almost four weeks prior. Clearly, she thought that following the teacher's directions was extremely important.

Khalidah also shared that even when working with her friends, she would finish her work before getting off task, though she also shared that she would sometimes get bored and walk around. When I asked her more about this, she made clear that she only got off task after finishing her work. The directions to finish work were more important to her than enjoying time with her friends.

Overall, Khalidah emphasized staying on task no matter the situation or with whom she was working. Staying on task allowed her to do well in class. Even though she valued her friendships, she seemed to more highly value staying attentive in class and doing what the teacher asked her to.

Shamshidah. Perhaps more clearly that Ohma or Khalidah, Shamshidah valued being compliant and staying on task. When I asked her what made her day go well, she answered, "In class I just... do what else the teacher says and just listening and do all work." Later, when I asked her if she would like more or fewer choices in school, she replied, "either one." Though she did not say much more directly speaking to staying on task, many of her comments had an implicit message that she would do whatever the teacher asked of her. 
Shamshidah did not say quite as much as Participants 1 and 2 about school in her country of origin (Malaysia). She did, however, describe clear differences between school in Malaysia and the United States. Like Ohma and Khalida, she attended a Muslim school. One difference was in the clothing; "Um, at school, we had to wear like... like uniform." Two other differences were in food and transportation; "Um, the... the food, we had to pay to eat. Um... we had to pay for the driver to get to school. We have to walk a mile to get to the bus."

Like Khalidah, she was surprised she had several different teachers in the United States. When I asked her what the differences were between school in Malaysia and the United States, she said of school in the United States, "We have to study religions. And... I'm good with English. The teacher, uh, like, for one hour for one person, and then hour for one person. Each day, like four or five teachers." She meant she was surprised that she had to move from class to class taught by different teachers.

Other differences that surprised Shamshidah included: “That we don't have to wear uniforms. The school is big. Um, we have to study a lot. We don't have to pay for the... bus... bus driver to get to school. We get free food.” Clearly, Shamshidah believed there were many differences between her school experiences in Malaysia and her experiences in the United States.

Like Khalidah, Shamshidah never spoke to any particular behavioral expectations, but her statements indicated an expectation of conformity and following the rules. When talking about her surprises, she indicated she was surprised she had so much freedom in schools in the United States. 
When it came to observations, Shamshidah worked hard to stay on task. At times, she worked too slowly to do exactly what the teacher was saying, but she never intentionally disobeyed the teacher. I only observed Shamshidah work with one other student, who was Caucasian American. Shamshidah barely talked during these interactions. Twice, she asked the student for clarification prior to the assigned activity. When she shared with her partner, she did so very quietly, pointing to he work rather than talking about it much. Her behavior was consistent with her interview statements. She only shared what was required of her and preferred to work alone. Compounding the situation was that the teacher reported Shamshidah's partner to be a very strong student. The fear Shamshidah had that others might be smarter than her was probably borne out in that class. Her partner likely intimidated her.

In one observation, she was supposed to calculate the area of six shapes and compare her answers with a neighbor. The teacher gave students about three minutes to complete each part. Shamshidah finished two of the problems, then asked her partner for help. Her partner quickly helped, then Shamshidah silently moved on to working on one more problem, until the teacher moved on to the next part of the lesson.

In another observation, students were asked to set up stacked equations adding or subtracting positive and negative decimals. After setting up the equations, the teacher asked students to check their equations with their partner prior to solving the problem. While many students began to work on their problems, Shamshidah asked two questions to her partner, then watched the teacher set up and solve a final example. Only then did she begin working. She spent the majority of the time allotted setting up three equations. 
Once she set up the equations, she spent approximately 30 seconds checking with her partner. It was unclear whether her equations were accurate, but it was clear that Shamshidah persevered slowly through setting up the equations and eventually checked with her partner as instructed. Multiple times during this sequence, the teacher reminded students to share their answers, but Shamshidah appeared to not follow directions by not sharing. However, she later shared once she finished the problems. She was just slow to do so.

In a stimulated recall session, while watching a video a clip in which the directions were to share her answer with a neighbor, Shamshidah said she really wanted to share her answer, but had to finish the problem first. At the beginning of the video clip, instead of sharing her answer, she was finishing the question. She worked quickly to finish the problem and shared her answer with her partner. Although she worked more slowly than other students in the class, she still tried to follow the directions of the teacher. After sharing one answer, she continued working on more problems so she could share others later. Her focus was on wanting to do what the teacher said, but she had a difficult time keeping up with the teacher's time constraints. Her difficulty keeping up with instruction could be at least partially explained by her ELD level. She was still learning English, and likely had some difficulty understanding exactly what she was supposed to do.

When I asked Shamshidah during a stimulated recall session how she would work with a student who speaks the same language as her, she said they would talk more. I followed up her answer by asking her if they would talk about anything other than math 
(the subject in which I observed her). She said they would still only talk about math. To her, the content was the most important part of the class, and she would not spend time distracting herself with other topics.

The only issue Shamshidah had with staying on task was not sharing with her partner when the teacher asked during an activity in which the students were setting up stacked equations. In stimulated recall, she reported wanting to finish the problems prior to sharing. She eventually shared, but not exactly when her teacher asked her to.

Overall, Shamshidah was very conscious of following the teacher's directions, even when she had difficulty with the work, and, thus, had a hard time doing so. Though she was surprised at some of the differences between school in Malaysia and the United States, she held on to the expectation that students should do whatever the teacher said.

Summary. One way the participants tried to ensure their understanding was to follow teacher directions. This value seemed to come from their experiences in their countries of origin, where compliance was emphasized. Even when it was difficult to follow directions, the participants nearly always stayed on task. While it was a way to ensure understanding, staying on task was important to all of the participants in and of itself.

For each participant, the major themes of seeking understanding and staying on task were both focused on their individual behaviors in their classrooms. As young adolescents, though, peer relationships were still important to the participants. They did not emphasize their relationships with others, but they believed it was important to be kind to their peers. 


\section{Being Kind to Others}

Coupled with seeking understanding and staying on task in their classes, the participants emphasized being kind to others. They generally preferred working by themselves, but knew working with others was an expected part of classes in the United States. While friendships were important to each participant, they were secondary to doing well in school. As such, they put high value on being kind to others as a way to ensure they did well.

Ohma. Ohma was very clear about her values when she said, "I like it when people, um, like, teachers, friends, and all the people are nice. And, like, funny, and, like, kind." Throughout her interviews, it was clear she valued having nice people around her and being nice to others.

Humor was an important part of her view of being nice. She gave an example of how teachers should act in describing her math teacher; "Whenever he tried to, like, say like, stuff, he like, yell it out loud and then it'll like make me laugh because I like get scared and then it'll like spoil the thing." By "spoil the thing," she meant the teacher would surprise her by being loud. She gave another example in saying a different teacher, "will say like different things than the class," meaning he would joke with the class. That same teacher would make her laugh when he would, "go to the food part and then make us hungry." Ohma highly valued the humor these teachers brought to their classrooms and she reported the classes they taught to be two of her favorites.

In addition to enjoying humor in the classroom, Ohma enjoyed spending time with her friends in school. When I asked her what she most liked about school, she 
answered, "Seeing my friends, and, like, having classes with my friends." Her focus on working hard and staying on task at school was integrated relatively well with her desire to spend time with her friends. She enjoyed working with her friends in school for school-related and friendship reasons. When I asked her why she worked well with her friends, she said:

Because they know me better than other people. They know that I don't like copying. I like, like, asking. And then, like, they are, like, smart, and, like, funny, so, like, I can stay with them. And, like, whenever I do something stupid, like, they will do something stupid and then we both will laugh.

Clearly, humor, friendliness, and kindness were key parts of her experiences with other people in school, whether with adults or other students.

To her, being kind included not getting other students in trouble, even if they were off task. When I asked her why she would not directly tell the teacher that other students were off task, she answered, “Because I don't want, like, other people, like, to get, like, hurt and, like, I don't want them to be sad at all." Her emphasis on not getting other students hurt carried through her interviews and observations, even with students she did not want to be around.

Despite not wanting to get other students in trouble, Ohma had perhaps the strongest views of other students among the participants. When I asked her about how students usually behave, she answered:

Sometimes, students don't work. They just copy off people and then they'll like, say to the teacher that they worked. And then like all that stuff. And then, we get 
BURMESE REFUGEE STUDENT INTERACTION

blamed when we don't have the stuff in our paper when we're working. The teacher will blame us, like, 'why aren't you writing the stuff that your partner has?' and like all that stuff.

The fact that other students copied clearly bothered Ohma, but she did not like to share her frustrations with the teacher. She had specific ideas of how to improve students' work. When I asked her how teachers should structure class, she shared:

They should sometimes, like, put the two, like, two copying person with two smart person. Like, so, like, it will get even. And, like, if... like, they would get even, and, like, they don't have to, like, copy too much, and like, we could teach them something, a little. And then they know something a little, and then, like, it'll get even.

I understood her statement to mean that students should help each other to not copy, and that it would be easier if they were placed in groups rather than partners.

For students who work hard, though, Ohma thought working in partners worked well. She gave one example:

Once, in my ELD 4 class... So, like, [Chinese student], this $8^{\text {th }}$ grader... I was having trouble doing with my, president essay. Ya, president essay. And then she started helping me. Like how she, um, she wrote it. So I, like, look at the first, second, then and, like, all those stuff, and then I started writing it.

When I followed up, she reported that the other student showed her the structure of the essay, which helped Ohma organize her writing. 
In observations, Ohma displayed the kindness toward others she emphasized in her interviews. She worked with many different students over the course of several observations. Never did I see her get outwardly frustrated, even when she was one of the students taking the lead on an activity and other students were not doing as much. The only time I saw Ohma frustrated was when she could not figure out what was in a film canister. Even though she was frustrated, she was still very friendly with her partner and another student she asked for help.

In stimulated recall, Ohma did not directly discuss the value of being nice to others. However, she said she did not like one partner in her group while rolling marbles under a box with pegs underneath because that student copied from others. Despite not liking her, Ohma said she was still nice to that girl because they were partners and had to work together to finish the assignment. She reported getting along well with everyone else I saw her partnered with.

Later, Ohma said it would be difficult to work with other Burmese students because she would have to help them too much, and, thus, would not get her own work done. When I asked her if she valued helping other Burmese students more than doing her own work, she replied, "I guess." I interpret this as an example of kindness, in that she would sacrifice one of her own values to help others.

Despite her strong views of other students who might copy, Ohma worked hard to be kind to everyone because kindness and humor were so important to her. She went so far as to not tell on other students who were cheating and act kindly toward a student she 
admitted not liking because she was supposed to finish the assignment. Clearly, being kind to others was something she valued highly.

Khalidah. While Ohma discussed the importance of being kind among adults and students, Khalidah did not share very much about how she viewed teachers, but emphasized her friendships with other students. She only had one thing to say about teachers, specific to her social studies teacher. She said of her teacher, "She doesn't get me sleepy." As I understood this comment, it was not a critique of her other teachers, but a complement specifically toward one teacher she believed especially cared about her.

It was clear she put more value on having friends in class than anything the teacher did. When I asked her what she liked about her favorite classes, she answered, "Um, fun people there." She also said, "I have friends in every one of my classes." While some students might have emphasized these traits as a way to get off task, Khalidah clearly was able to value having friends in class while also seeking understanding and staying on task.

One possible reason Khalidah valued friends in school so much was that she did not see her friends outside of school very often. When I asked her if she sees her friends outside of school, she replied, "No, not really." School was an important place for her to make friends, because aside from Ohma, she reported that she met all of her friends at school. When I asked her specifically how she met her friends, she replied, "[Ohma], I used to live with her in um, one of our old apartments. And the other ones I met at school." She seemed to still want to make more friends during class. I asked her what 
she liked about working with other people and she answered, "Um... I don't know, making friends. Um... getting to know each other."

Khalidah did not report as strong of negative opinions of other students as Ohma, but still indicated some level of mistrust that other students would actually work when describing what might happen when a teacher chooses partners. She said:

He doesn't really know if we have something going on with each other. Like, maybe we had a fight or something, or an argument, and he just puts us together, and, like, we're not really learning, so I think it's better to, like, have a person choose their own partner.

In my experience as a teacher, this is not an uncommon sentiment among students who want to talk with their friends rather than work. With Khalidah, however, her actions as were consistent with a student who actually worked better when choosing her own partners.

She confirmed her interest in actually learning when explaining why working in small groups was preferable. She said:

If it's, like a bigger group, some of them, they might not like you. They work by themselves. And, like, if it's, like a smaller group, then you could like, interact with each other. You could say things, like, share your details and things. Taken together, Khalidah seemed to have a preference for working with other students over working by herself, but she also indicated a preference for working with smaller groups rather than large groups. Either way, she indicated trying to get along 
with other students was important to her, especially in finishing her work and understanding the material.

During observations, Khalidah worked well with several different students. In one observation, she worked with a Nepali female friend during class. They were tasked with identifying the main events from a novel, which they were able to do together. Khalidah did most of the talking, but they worked well together. During a different observation, Khalidah worked with a Latina female friend to identify events leading up to the Civil War. They worked very well together to finish the assignment in less than the allotted time. Though they got slightly off task at the end by bouncing an eraser on the floor, it was not disruptive to the rest of class.

In other observations, Khalidah was very helpful to her partners. In one instance, when working with a Hispanic male who had very little of his assignment completed, she tried to coach him through it. She kept being nice to him even when he was distracted and off task.

One interesting note is that despite her statements indicating she preferred to work with other students, Khalidah did most of her actual work alone during observations. After she finished her work, she would then check it with other students or help them finish their own work. It was as if she wanted to work with other students only to check the accuracy of her work, not to help her generate ideas.

Khalidah also did not say very much about being nice to other students during stimulated recall sessions. However, she said every female she worked with was her friend, implying she valued being nice to others. Later, she said that the students she 
worked with during the observations were only her friends in school, but another student in the class (who was Iranian) was a friend of hers outside of school.

Khalidah said when another student does not talk very much, she does most of the talking. The way she discussed the amount of talking that happens in interactions, she seemed to imply she thought there was a certain amount of talking or interaction that should happen in an interaction. If the other person initiated the interaction, they would do most of the talking. If the other person did not initiate the talking, Khalidah would.

Like Ohma, Khalidah said during a stimulated recall session that working with students from other cultural backgrounds was easier than working with students from her own cultural background. After watching a clip of her working with a Caucasian girl, she said working with students from any cultural background would not have made a difference in how she worked. I asked if she would work equally well with Burmese or Rohingya students. She replied that she would be more likely to get off task and talk about non-school related things if partnered with a student from her own cultural background.

While discussing an interaction with a Hispanic girl, Khalidah shared an interesting story about what usually happens in class. She said the Hispanic girl she was working with at the time usually finished her work first, then helped an Iranian girl, who helped a Nepali girl, who then helped Khalidah. I did not observe a situation like what she described, but I only saw her work with one person at a time.

In sum, Khalidah was very peer-oriented, getting along well with every student with whom she was partnered. She enjoyed working with friends, but was willing to 
work positively with anyone. Even when the students were distracted, she acted nicely toward and had a generally positive opinion of them.

Shamshidah. Though she did not speak at length, Shamshidah strongly emphasized the importance of acting kind toward others. She described smart students as, "Kind, respectful, responsible." When I asked her what she thought of her friends, she said, "They're friendly sometimes. They're nice to people." Several other times, she mentioned a desirable trait as nice or kind.

Though she reported having friends at school, she did not share much about them. When I asked her how many friends she had in her classes, she said, "Um, maybe four or five, six." When I asked her how she made friends, she named only one friend and said, "She's kind. She's nice." When I asked her how they met, she replied, "I saw her at school and I say, 'hi'. And then we became friends. So we hang out together, talk." I asked her if she talked to her friends most in class or at other times during the day. Shamshidah replied, "At lunch." It is possible that because she was still learning English, she did not have the vocabulary to tell me about more of her friends. Whether or not she had more friends, it was clear that being nice was an important trait of her friends.

Not all of her interactions were with kind students. Like Ohma, Shamshidah encountered situations in which other students were not doing what they were supposed to be doing. And like Ohma, Shamshidah would not directly tell the teacher when other students were off task. When I asked her why, she said, “Because I don't want to.” After I pressed her a little more for a more specific reason, she clarified, "It would make more problem." I asked her to clarify that statement and she indicated that other students might 
get mad at her, though she could not think of an example of when a student actually got mad at her.

Despite her emphasis on being nice to others, Shamshidah reported not liking to work with other people at all. When I asked her if she preferred working by herself or with other people, she answered, "I just like to work myself, so it's more comfortable." When I followed up to ask her why she preferred to work by herself, she answered, "I feel nervous." I asked her why and she answered, "When the person is... doesn't friends with me. They're people who I don't know." I continued to follow up, and Shamshidah stated, "Some people might be smarter than me." She then offered two examples of when students did something that made her feel not as smart as them. For the first example, she said, "Like we have in three groups. We've got three people. Um, like, one person just talks with them, doesn't talk with me." For the other example, she said: In science class, me and there's two girls. So we are in groups. We are doing some projects. So, we have me, she, and she's a girl so she don't talk with me. She just talk with the people. Like to her friend, just her friend. So she just talk with her, you know. Like when we were doing some projects together. She don't talk to me, at all.

It was interesting to me that both examples of when Shamshidah felt less smart than others involved other students not talking to her. However, she also reported feeling most comfortable working alone. She was not able to directly reconcile this conflict. However, when I asked her who helped her when she needed help, she replied, "They 
always help me." I inquired who she referred to when she said, "They," and she indirectly indicated she meant any student seated near her in the following exchange:

Investigator: How do you ask for that [help from other students]?

Shamshidah: 'Can you explain for me?'

Investigator: And what's their response, usually?

Shamshidah: They say, 'yes,' and they just explain for me.

Overall, Shamshidah seemed to want to work alone because of her shyness, but also appreciate the help available when she worked with other students. Her sentiment was similar to that of the other participants.

Observing Shamshidah was interesting. She was only ever partnered with one other student, a Caucasian American female who was very good in math, but very quiet and tended to keep to herself. Shamshidah frequently asked her questions and was always very polite in doing so. I never saw an interaction I would describe as explicitly kind, but the two were always congenial despite them both seeming aloof.

Shamshidah also did not say anything directly during stimulated recall sessions about being kind to others. However, when I asked how her interactions might look different with different partners, she strongly indicated she would still be friendly with other students by saying she would treat them all the same way.

Shamshidah claimed the cultural backgrounds of other students did not affect how she worked with them, but for different reasons than the other participants. During a stimulated recall session, she directly said she did not like to talk very much. She preferred to keep her interactions short and focused on the topic at hand. Even during 
interviews and stimulated recall with me, she rarely elaborated; her answers were almost always one sentence or fewer.

The only time Shamshidah said interactions might look different, depending on the cultural backgrounds of students, was if her partner spoke the same language as her. She could not, however, explain how the interactions would look different. She said they would still only talk about the class they were in. I inferred from her answer that, perhaps, she and her partner would be able to explain their thinking more easily, but would still stay on task.

When we discussed working with her partner, who also did not talk very much, Shamshidah shared that she would be afraid if she had to work with a student who liked to talk a lot. Because she was shy around others who talk a lot, she preferred to work with others who were quiet.

Even though Shamshidah preferred to work alone, she was still invariably nice to her partner when they were supposed to interact. Combined with her emphasis on kindness from her interviews, I conclude she viewed being nice as roughly on the same level as being compliant when it came to success in schools in the United States.

Summary. Being kind was a strong value of all three participants. They valued others being nice to them, and they were nice to others, even when they did not have to be. They seemed to understand that being nice to other students would help them do well in school. In addition, they did not want to cause problems between other students, so even when students were doing something they should not, the participants would not tell the teacher. 


\section{Connect to Research Questions}

According to Moustakis (1994), one goal of a transcendental phenomenological study is to describe the participants' noema (experience), which is what I did when describing the themes. Another goal of transcendental phenomenological research is to describe the participants' noesis (interpretation of experiences). Using the themes to answer the research questions accomplishes this goal. Below, I use the information in reporting the themes to address each research question, attempting to explain the participants' interpretations of their experiences in their multicultural middle school.

Experiences of Burmese students in multicultural middle school classrooms. In response to Research Question 1 (What were the experiences of Burmese refugees in multicultural middle school classrooms?), I found that participants saw school as a place to learn. While they each had friends, and friendships were important to them, once they arrived in class, the purpose was to learn and stay on task.

Ohma. Ohma seemed to experience school as somewhat of an outsider. Though she had friends and enjoyed laughing and having fun in school, she spoke of her experiences with a dichotomous view with her being different from others. Some of her experiences of being different from others may have come from her life at home. She spoke of her family as "villagers" who, "eat on the floor" wash their close with their hands instead of going to the laundry. She was not very family oriented; when I asked if she spent time with her family, she replied,

Not really. I just like... if they tell me to do something, I do it. But, like, when 
they tell me to do nothing, I try to like be myself... by myself, or do my homework, or go to my... go to the other side and play.

As the oldest child in her family, she seemed to want to get away from the younger children. Though she clearly cared for her family, she seemed to view them as more primitive than her, and she was trying to reorient toward her friends.

As much as she was reorienting away from her family and toward her friends, she did not talk about school as if she fit in with her peers. In school, she believed many other students did not work hard, whereas she did. Though she viewed herself as different from most other students, she also did not want to ostracize herself by pointing out to a teacher what others may have done. Telling on other students, according to Ohma, would create more problems for her. Instead, she preferred to focus on the task at hand and try to learn as much as possible.

Khalidah. Khalidah was more social than either Ohma or Shamshidah, but still viewed school as a place to learn and work hard. Her social nature seemed to extend to her home, as she reported being close to her family and neighbors. When I asked her about her home life, she said, "We're nice. We hang out with each other a lot mostly. And then, like, we have neighbors. We usually play outside with them." Describing what she does with family, friends, and neighbors, she said:

We go to Lincoln Park on like... on the evening when my dad doesn't have work or anything. My baby sister and. we go to like rivers. Sunriver, Blue Lake, and... Last Monday I think, or was it Friday? I don't know. We went on this picnic, it's from mosque and they congratulate us. There's like different type of country feel, 
like, mostly from Burma though. And there's like... they give prizes for, like, the people who read good. Ya. It was fun. I was hanging out with Ohma and my sister and [ a former student who is Burmese] who I didn't mention before.

She seemed to enjoy spending time with her family and friends outside of school, so I did not get the sense she was using school as a way to escape. That being said, her younger brother died tragically in a drowning accident about one and half years before the study. Khalidah (and her entire family) was understandably devastated. One has to wonder if she was seeking out attention, acceptance, and distraction to deal with any possible feelings of loss.

Whether or not Khalidah was covering up painful feelings, she had a clear idea of how to ensure she learned what she was supposed to and was successful in school. At times, she valued working with other students, whereas other times, she worked very well on her own. As stated earlier, she completed her work during observation sessions more quickly than her partners. She then helped them finish their work. Though she never stated it directly, it seemed as if she knew that helping her partners with their work would help her learn the material better.

More so than the other participants, Khalidah was very proud of her academic progress. She openly bragged about earning an award in her Read 180 class, and she even mentioned getting a prize for reading well in the picnic referenced above. She had strong memories of struggling with reading and took great pride in how well she was doing at the time of the study. 
Shamshidah. Shamshidah was the most serious about school of the three participants. At the same time, she was the least academically successful. Though she was a very hard working, conscientious student, she struggled to keep up with the pace in her math class. Likely, her struggles stemmed from where she was in acquiring English. In her interviews, it was clear she had difficulty expressing some of her thoughts without long pauses and thinking deeply about exactly what she wanted to say.

Whereas the other participants may have used school to see friends outside of their family, Shamshidah did not seem to put much emphasis on friends at school. She seemed very close to her family. When I asked her what she did outside of school, she replied, "I just play with my sisters. And we watch TV, and we eat together. That's all." When I specifically asked about her friends, she said, 'Um, my friend is [her friend's name]. And she's kind, she's nice. And... about my family?" She proceeded to say, “Um, I have six family, six people. And I have four sisters, three sisters. They are... I'm the oldest. They are the youngest and, we live together. We live in apartments." Even when I asked her to speak about her friends, she turned the conversation toward her family.

Echoing her reluctance to speak about other people not in her family, she was clearly nervous around other students. She said several times that she was shy and did not like to work with other students. In observation sessions, she was reluctant to talk to her partners, only doing so when the teacher asked her or when she needed help. Part of her reasoning for not wanting to work with other students was her belief that she was not as smart as other students. When I directly asked her why she did not want to talk to or 
work with other students, even when they were nice, she said, "Some people might be smarter than me."

Despite her reluctance to work with others and lack of confidence in her intelligence, Shamshidah had a clear vision of how she should behave and what she needed to do to be successful. She placed a high emphasis on staying on task, and did not let anything distract her from focusing on learning so she could be the best she could be.

Experiences of Burmese students in intercultural interactions. In response to Research Question 2 (How did Burmese refugee students experience interactions with students from other cultural backgrounds?), I found interactions with other students were very focused on the task at hand. Because they were in class to learn, they did not spend much time socializing during class. Even so, they worked well in every pairing and grouping I observed, indicating a general belief that working well with others could lead to success in the classroom.

Ohma. Ohma approached interactions with other students cautiously. She had a mistrust that other students were actually working, but was still willing to work through the activities because of her dual foci on learning and staying on task. She gravitated toward working with those she believed were good students, but put her full effort into completing tasks even when partnered with students who she thought were not doing their best.

In the interviews, Ohma clearly stated her view that some students were not at school to learn. Instead, they just copied work from other students so they could get a good grade. She said, “Sometimes, students don't work. They just copy off people, then 
they'll like, say to the teacher that they worked." Ohma did not want to work with those students, but still would when she had to. In one interview, she went so far as to say she wanted to help them. When I asked her how teachers should group students together, she said:

Um, they should, like. They should sometimes, like, put the... two, like, two copying person with two smart person, like, so, like it will get even. And, like, if... like they would get even, and, like, they don't have to, like copy too much, and like, we could teach them something, a little... and then they know something a little, and then, like, it'll get, even.

In class, she displayed her willingness to work with any student, regardless of her feelings about them. In one activity, she worked with a student she identified as one who copies. Though Ohma did not interact with her very much, she was consistently nice to her and made sure the group finished their work and understood what they were supposed to learn.

Another time in class, she asked a friend from Nepal for help. As stated earlier, I suspected they might get off task because I had seen them be silly in the hallway and the teacher was on the other side of the room. Instead, Ohma used this interaction to ensure she not only finished her work, but understood what she was supposed to learn.

Khalidah. Khalidah clearly stated her belief that working with other students would help her learn more. She enjoyed working with other people for reasons not necessarily related to school. When I asked her why she enjoyed working with other 
people, she responded, "Um, I don't know. Making friends. Um, getting to know each other." She enjoyed working with other students in small groups. She reasoned:

Because, like, if it's, like, a bigger group, some of them, they might not like you. They work by themselves. And, like, if it's like a smaller group, then you could like, interact with each other. You could say things, like, share your details and things.

She was clear that working with other people helped her learn as well as make friends. As stated earlier, she said, "The more I work with people, the more I get in my head. And that helps me think more." She also said, "When people talk or things, it comes. It kind of gets me to think more. I don't know why how that works, but I just think more when peoples like working it together instead of, like, individually."

Her desire to work with other students paid off in her learning. During observations, she was consistently ahead of other students on her work and helped them finish. She said this was not always the case; sometimes other students helped her, but she was always the one who was more on task than other students in class. She said that two other students in particular who were cousins would shut her out during work time. Though she did not like how they treated her, she tried to stay focused in class and finish her work.

Aside from the cousins who would refuse to work with her, Khalidah seemed to enjoy working with any other student. In an interview, she said as much in stating, "I'm okay working with anyone.” Even with her annoyance at the students who would not work with her, Khalidah did not get mad at them. She said they got mad at her instead. 
When she was having conflicts with other students, Khalidah preferred to choose with whom she worked. When teachers choose partners, she said:

Sometimes he chooses our partner and, like, he doesn't really know if we have something going on with each other. Like, maybe we had a fight or something, or an argument, and he just puts us together and, like, we're not really learning so I think it's better to, like, have a person choose their own partner. Interestingly, her desire to choose her own partner was rooted in her value of seeking understanding of what she was learning. When I clarified why she wanted to work with her friends, she said she would learn more by working with them rather than other people.

Shamshidah. Unlike Ohma and Khalidah, Shamshidah did not enjoy working with others. Interactions with other students seemed to make her anxious. She much preferred to work alone. However, she obviously benefitted from working with others.

Shamshidah said she had friends at school, but almost never worked with them in class. She only talked with them during lunch, even though she had friends in some of her classes. One reason she did not socialize with her friends in class was she was so she could focus on her work and learn as much as possible. Another reason was he belief that other students were smarter than her.

Shamshidah indicated several times she believed she was not as smart as other students. Despite her belief that she was not smart, she worked very hard to do well in school and work with other students when necessary. In fact, she acknowledged that other students could help her learn better when she said, "They always help me." In 
observations, Shamshidah often asked for help from her partner. Even though she believed working alone was better than working with other students, she certainly benefitted from working with peers.

\section{Effects of intercultural interactions on Burmese students' experiences in}

multicultural middle school classrooms. In response to the Research Question 3 (How did the interactions Burmese refugee students have with students from other cultural backgrounds affect their school experiences, including learning and identity formation?), I found that all three participants seemed to use the experiences they had in their home countries to inform how they should behave in the United States. In all cases, they spoke of the importance of following directions and being compliant. In the United States, their perceptions helped them work well with other students to focus on understanding what they were learning and be successful in school. In school in the United States, their existing habitus of focusing on understanding and trying to be successful helped them to work effectively with students from various different cultural backgrounds. Working successfully with a variety of students reified their identities as good students.

Ohma. Ohma did not speak at length about her experiences in her country of origin. Listening to what little she said, though, her experiences in Burma seemed to have a strong impact on her. Unlike the other participants, she actually lived in Burma for some time. Speaking of her upbringing, she said, "My parents came from Thailand. They met at Thailand and they born me at Thailand. But then I moved to Burma when I was two, and I grew up there." Though she did not remember much about her time there, she remembered having to follow directions. If she did not, she would have been 
punished harshly. She did not seem to fear harsh punishment in the United States, but she certainly valued following teachers' directions.

In following teachers' directions, Ohma held herself up to be one of the good students at school. She did not copy, she stayed on task, and she tried her best in school. Other students did not work very hard. Even though they did not do what Ohma thought they should, she was willing to help them. In the group activity where they were rolling marbles to find the location of pegs under a box, she even worked well with a student she identified as one who copied off others.

It certainly seemed as though Ohma viewed herself as a good student amongst many students who did not do what they were supposed to. In some ways, her belief that some students were good and others were bad solidified her stance as a good student. She followed teachers' directions, she focused on learning, she was willing to work with anyone, and, consequently, she did well in school.

Khalidah. Like Ohma, Khalidah did not speak at length about her experiences in her country of origin. What she said, though, was similar to what Ohma said. Being compliant was especially valued, and not following directions was punished rather harshly. She said of what would happen if students did not follow directions: "Um, if you don't do something they tell you to do, they like hit you with a stick." Unlike Ohma, and despite the harsh consequences, Khalidah spoke fondly of her school in Bangladesh. She attended an Islamic school, where classes were very teacher-centered and students were not allowed to work independently. She described the experience when she said, "The teacher, she works with us to, like, the whole class period." She clarified by saying, 
"We worked as a class actually." Her experience, then, in the United States has been quite different than what she was used to in Bangladesh. As for her preference, Khalidah was equivocal:

I think I'm stuck in the middle. I want to be in my old school because, like, we worked, like the whole class and, like, do what we, like sing and things. But here it's more like friends and things, so... I'm stuck actually. I can't choose. Whatever her preference, Khalidah saw herself as a student who did what she was supposed to do and worked well with others.

As stated earlier, Khalidah had a clear vision that working with other students helped her learn more. In working with others, she saw herself as a leader. While seeing herself as a leader, she spoke of being willing to accept help from others. In every observation session, she had finished more work than her partners, but she said in interviews and stimulated recall sessions that she often asked for help from classmates. Clearly, Khalidah viewed working with other students as a way to make herself a better student.

When it came to how she saw herself as a student, Khalidah was very proud of her accomplishments. She was keenly aware of her struggles acquiring English, and celebrated how well she has done in school. Khalidah did not view other students as negatively as Ohma, but she seemed to pride herself on being a better student than most of her peers.

Shamshidah. Of the three participants, Shamshidah was the most serious about her role as a student. She saw school as a place to learn; socializing was not a priority. 
Though she had friends in some of her classes, she did not speak about school as a place where friendships were important. Though she struggled to understand what she was learning, doing well in school was extremely important to her.

When Shamshidah spoke of school in Malaysia, she focused on how much she had to follow directions and how it was a challenge to get there; she had to wear a uniform and pay for a taxi to get her to school. Shamshidah did not share many details about school in Malaysia, but she indicated it was very regimented.

By contrast, she had a lot of freedom in school in the United States, which presented a challenge in adjusting to what she could and could not do. Many features of school in the United States surprised her. While in school in the United States, she seemed to follow directions as if it were regimented like in Malaysia. She put a high priority on following directions and focusing on her learning. She would work with other students when asked by a teacher, but preferred to study on her own. She did not like to read or write, but still thought it was important to learn more.

Shamshidah did not see herself as a particularly good student, but she was confident that she eventually would be if she kept following directions and working hard. Sometimes, working with other students made her feel not as smart as them, which she was very nervous about. However, she was willing to accept help when she needed it. I saw her ask for help several times during observations. She was also willing to help other students when necessary, which she seemed to feel empowered by, but it did not happen very often. 
Though Shamshidah reported liking school very much, and she saw herself as a good student when it came to following directions, she was nervous about her academic ability, especially when working with other students. Despite her reservations, she was confident that her focus on school would lead her to success in the future.

\section{Interpretation of Findings}

The participants, Ohma, Khalidah, and Shamshidah, were all trying to navigate the norms a relatively unfamiliar school system by doing what they thought would make them successful. They sought out understanding in their classes, put a high priority on staying on task and following teacher directions, and tried to be kind to other students. Before discussing my interpretation of the participants' experiences in more depth, I first bracket out my experience to describe the experiences of the participants.

\section{Bracketing Out the Researcher's Experience}

In a transcendental phenomenological study, the researcher needs to bracket out his or her experience prior to interpreting the experiences of the participants (Moustakas, 1994). I initially approached this study as a teacher interested in the experiences of a select group of my students. For the study itself, I took the lens of a researcher rather than the teacher of the students. To do so, I observed the students in classes I did not teach, and I met with them for interviews and stimulated recall sessions during mutually agreeable times outside their classes (lunch and after school).

My background is quite different from the backgrounds of the participants. I was born and raised in a rural small town in the Northwestern United States. The town in which I was raised was almost entirely bicultural; English and Spanish were the only two 
languages I heard while growing up. As a white male, I did not have to navigate unfamiliar cultural norms. Though I traveled to Europe, Mexico, and Hawaii during high school, I did so with my family and mostly stayed at resorts catering to tourists. As a result, I had little experience in multicultural contexts until college.

After high school, I attended a small, liberal arts college in the Northwest. Though the school I attended had some cultural diversity, it was not nearly as diverse as the school the participants in this study attended. It was during college that I had my first real experience with diversity; I traveled to Jordan to participate in several archaeological projects. I spent approximately two months each during three separate summers in Jordan. During the first summer, I mostly stayed in the capital city, Amman, where I assisted with stone tool analysis. During the other two summers I was mostly in a sparsely populated area near the Dead Sea assisting with excavations and stone tool analysis. During those summers, I had my first experiences of being a cultural outsider. Granted, as a white male, I was not as much of an outsider as many people, but I got a taste of what it was like to live in a place where I was unfamiliar with the language and many of the cultural norms.

After my time in Jordan, I spent two years working in rural elementary schools in the Pacific Northwest as an AmeriCorps member. In one of those schools, I worked closely with a group of students who were the children of migrant workers. That was my first experience with the challenges many students face in schools in the United States. Most of the students I worked with did not speak English fluently, were unfamiliar with 
the norms of the area in which they lived, had unstable home lives, and struggled academically. It was because of that experience I began teaching.

My first teaching job was at the school where the study took place. I sought out a teaching position at that particular school specifically because of the diversity. I had a strong interest in learning how to educate students who come from difficult backgrounds, including those who live in poverty and who speak languages other than English at home. I conducted the study described in this paper during my ninth year teaching at the school.

During the study, I was working as a full time teacher and gathering data during times I was not teaching. Playing dual roles meant I had to quickly transition my focus and my role from teacher to researcher. It also limited the opportunities I had to see the participants in various contexts. Because I could not observe each participant in various contexts, I chose to observe each participant in a different content area; I observed Ohma in science, Khalidah in social studies, and Shamshidah in math.

Separating my teacher lens from my researcher lens was difficult. I was the social studies teacher for two of the participants while gathering data, and I was the social studies teacher for Khalidah during the 2013-2014 school year. Though there was likely a power differential that may have intimidated the students, I tried to temper it in a variety of ways. I assured them that their participation and comments about school would have no effect on their grades or treatment at school. I spent quite a bit of time helping them feel comfortable in interviews, observations, and stimulated recall. I did not begin interviews until they said they were ready. I visited their other classrooms several times prior to filming. I let them choose the times we had interview and 
stimulated recall sessions. Perhaps most importantly, I had a relationship with the students prior to the study beginning, and I kept a relationship with them after the study concluded. In the classroom, I have long prided myself on not being an authoritarian teacher, which has helped students feel very comfortable around me. I am confident the participants were comfortable throughout the process because of my approach to education and the time I put in to working with them.

Even though there were some drawbacks to my having dual roles as a teacher and researcher, there were some benefits as well. First and foremost, I was around the participants much of the time during the study. From 7:30 a.m. to 3:00 p.m. Monday through Friday I was at the same school as the participants. Though I had limited periods of time to conduct the actual study, I was present in the lives of the participants as a teacher in the school. During the days, I would commonly greet them in the hallway, check in with them to see how their days were going, and do anything else I could to make sure they felt comfortable in the school. To be clear, I did not do these things only because they were participants; I have always tried to greet, check in with, and ensure comfort for every student in the school. Doing so has allowed me to build strong relationships with students throughout my teaching career, even those who I did not teach.

In addition to building strong relationships with all of the students at my school, I have been connected to the Burmese refugee students in my school for several years. In the summer of 2011, I taught a summer school class for students learning English. Four of the five students in the class were Burmese refugees, and they spoke four different 
languages. At the time, I knew very little about Burmese refugees. In the class, I asked students to create a small project about their cultures, from which I learned a great deal. Since that experience, I have been fascinated with the journeys of Burmese refugee students. I have taught at least one student who was classified as such every year since. In the 2012-2013 school year, I conducted a small study for a graduate class in which I interviewed four Burmese refugee students about their experiences. One of the four participants was the older sister of Khalidah. Two of the other participants were sisters who used to live with Ohma and Khalidah. After so many years in contact with students from the Burmese refugee community, I think I have built enough rapport with the students in general that they felt very comfortable talking with me.

\section{Experiences of the Participants}

As individuals, each participant had different experiences, but they all had some things in common. They all sought out understanding in their classes, valued staying on task, and tried to be kind to others. With these three actions, students embodied their views of what good students should be.

The participants in this study experienced many of the challenges Burmese refugees typically face (Dávila, 2012; McBrien, 2005). All three were born outside of Burma, moved to the United States at a young age, and have moved within the United States. All were in the program for English language learners. They lived in a neighborhood where few other people spoke their languages. They attended a large, multicultural middle school where the vast majority of students lived in poverty. 
In school, the participants engaged in intercultural interactions reluctantly and with a strong focus on education. In a highly multicultural middle school such as the one attended by the participants, nearly every interaction was intercultural. In class, students did not actively seek out intercultural interactions. Because they were so focused on learning and following the teachers' directions, they only interacted with students from other cultural backgrounds while doing the work assigned by the teacher. Even in the instance Ohma was working on a different assignment than the rest of class due to it being lost, she only interacted with her friend to get clarification on how to do it.

Despite their reluctance to engage in intercultural interactions, participants did so effectively with a range of students from other cultural backgrounds. They reported working well with all students. In observations, I saw the participants working equally well with all the other students with whom they were partnered. Many of the interactions were short, but there was only one instance in which any of the participants (Khalidah) was off task. That instance occurred after she and her partner finished their assigned work.

The participants had mostly settled on identifying as good students. Though they had settled, they also hinted at exploring other possibilities. Because they identified as good students, the participants would best be described as in Marcia's (1980) foreclosed status, in which ethnic minorities settle on an identity without exploring other possibilities. The participants had settled on being good students seemingly without exploring other options. The way the participants talked about their identities as students, 
being anything other than good was not an option. Other students might have been bad students, but the participants were not able to think of themselves in that way.

It is probable the participants were engaging in identity formation outside of school. Each participant described her home life very differently than the other two. Ohma said she spent a lot of time with her family and people she knew from her mosque. Khalidah said she spent a lot of time with her friends. Shamshidah said she spent most of her time with her immediate family. Because the participants were in early adolescence, when people tend to shift their orientations from family to peers (Erikson, 1950, 1968), it makes sense that they reported different levels of orientation from family to friends. Shamshidah was very family-oriented; Ohma was family-oriented but spent quite a bit of time with friends also; Khalidah was very friend-oriented. I view the differences as both indicative of the students being adolescents and as displaying the range of individual differences between students.

Despite differences in friend-orientation outside of school, the participants were consistent in their foci in school. They stayed on task, worked hard to understand what they were learning, and were kind to others. Those were ways to be successful in their home countries, and the participants applied those successful behaviors in their schools in the United States.

\section{Limitations of Study}

No study is perfect (Maxwell, 2013). In any study, there are limitations that reduce the readers' confidence in the results. In the study I conducted, the limitations included few participants, limited time, limited classes in which to observe, potential 
influence by a teacher being the researcher, possible fabrication by the participants, and limitations inherent in the methods chosen.

\section{Few Participants}

Three students agreed to participate in my study. Conducting the study with only three students did not offer me the opportunity to access a wide range of individual experiences. However, because I conducted a qualitative, transcendental phenomenological study, it was not my goal to have a large sample size from which I could generalize. A small number of participants is a hallmark of qualitative studies (Plano Clark \& Creswell, 2010), and transcendental phenomenological approaches in particular (Moustakas, 1994). Alitolppa-Niitamo (2004) emphasized that the experiences of immigrant and refugee students should be examined through the context of each individual's unique experiences. Though I did not gain a wide perspective from many participants, I was able to deeply explore the experiences of a few students to try and understand them as best as I could.

\section{Limited Time}

Throughout the study, I worked as a full-time teacher. My schedule allowed me to have one 83-minute period each day during which I was not teaching. Because I made the decision to conduct the observations outside of my classroom so as to limit any potential undue influence, I could only observe during my non-teaching period, meaning I was unable to see the participants interacting with a wide range of students from other cultural backgrounds. I could not ethically remove the participants from class, so I conducted interviews and stimulated recall sessions during lunch and after school when 
the participants had free time and agreed to meet with me. As such, I had to limit the length of each meeting, which meant I could not give the participants unlimited time to discuss their experiences.

I also was not able to begin the study until about eight weeks prior to the end of the school year. I collected all of my data within those eight weeks. With more time, I may have achieved a deeper understanding of the experiences of Burmese refugee students, but logistics did not allow for unlimited time for that type of exploration.

\section{Limited Classes}

In addition to having limited time, I had limited opportunities to observe participants interacting with students from other cultural backgrounds. The period during which I did not teach was the last 83 minutes of each day. I could only observe participants in class during this time period. Because of how students' schedules were created, I could only observe Ohma in science, Khalidah in social studies, and Shamshidah in math.

Even though I could only observe each participant in one class, I was able to view a variety of different content areas in which Burmese students interact with students from other cultural backgrounds. Even though I saw students in three of four core content areas (I only missed Language Arts, in which students tend to not interact with others as much as in science, social studies, or math), I did not see the participants interact with a lot of different students. In total, I saw participants interact in class with 10 different students from seven different cultural backgrounds. Though seven was enough that I was able to see the participants' patterns in their interactions with other students, it pales in 
comparison to the 35 or more cultural backgrounds represented among the students at the school the participants attended.

\section{Influence of the Teacher}

Seidman (2013) specifically warned against teachers interviewing their own students due to the power differential that could affect the students' answers. Partially for this reason, I chose to conduct observations outside of my classroom. In doing so, I limited my influence by allowing the participants to see me as a researcher and explorer rather than a teacher. Despite trying to separate my roles as teacher and researcher for the study, I could not entirely eliminate my influence on the participants as a teacher.

During the study, I taught social studies to two of the participants. The year before I conducted the study, I was the social studies teacher for the other participant. No matter how much I attempted to mitigate any potential influence as a teacher, I still held that role for students.

\section{Potential for Fabrication}

I approached my study from a constructivist paradigm (Guba \& Lincoln, 2005) and used a transcendental phenomenological approach (Moustakas, 1994), meaning I let each participant tell their own stories as they saw them. With such an approach, the potential existed for students to fabricate their experiences. To limit fabrication in interviews, I conducted observation and stimulated recall sessions. Even though triangulation can limit bias and potentially increase validity (Maxwell, 2013), I cannot be entirely sure the participants were not putting on acts in my presence. If the participants 
were fabricating their experiences and acting in my presence, they were extremely consistent in doing so.

\section{Limitations of Methods}

Though the three methods I chose captured the experiences of Burmese refugee students in a multicultural classroom from their perspectives, each method came with limitations. In interviews, especially semi-structured interviews, there was a risk of the questions I asked influencing the answers and skewing the participants perceptions of their experiences (Vogt et al., 2012). In observation, I had to be careful about what I chose to notice. According to Vogt et al. (2012), researchers can easily become overwhelmed if they do not choose strategic ways to observe. In stimulated recall, I had to balance the parts my participants thought were important with the parts I wanted them to notice. 


\section{Chapter 5: Discussion and Conclusion}

In the previous chapters, I explained that refugee students enter the United States facing a daunting set of challenges, from unfamiliarity with the hegemonic culture and language to living in poverty (McBrien, 2005). Burmese refugee students are one of the largest recent groups of refugees who have entered the United States (Martin \& Yankay, 2012; UNHCR, 2015). One way these students could overcome these challenges is through intercultural interactions (Gurin et al., 2011; Ryan \& Patrick, 2001; Wentzel et al., 2012; Yoon, 2012). In the school where I conducted the study, refugee students had ample opportunities to engage in intercultural interactions due to the diverse student body. There were students who spoke over 35 different languages in the school, making the context super-diverse (Vertovec, 2007). Unfortunately, Burmese refugee students typically did not engage in many intercultural interactions. The purpose of this study was to examine the experiences these Burmese refugee students had in their multicultural middle school, especially in light of the benefits they could access through intercultural interactions. In exploring the participants' experiences, I investigated three research questions:

1. What were the experiences of Burmese refugees in multicultural middle school classrooms?

2. How did Burmese refugee students experience interactions with students from other cultural backgrounds? 
3. How did the interactions Burmese refugee students have with students from other cultural backgrounds affect their school experiences, including learning and identity formation?

As my theoretical framework, I used Bourdieu's (1973, 1977, 1990; Bourdieu \& Wacquant, 1992) theory of field and theory of social and cultural reproduction. In these theories, people navigate certain fields by learning and replicating the habitus that are accepted in those fields. People whose dispositions most closely match the habitus of the field have more power than others. Their dispositions, in turn, shape the habitus of the field in which they operate. In the context of this study, Burmese refugee students, to be successful, would have to learn the norms of the classes they attend. Because of their marginalization, one way they could learn the norms of the school could be through intercultural interaction, which they were easily able to do in their school context. While using Bourdieu's theories to frame my study, I remained cognizant of Yosso's (2005) critique about marginalized students bringing their own types of capital with them that also helped shape the field.

In middle school, adolescents are typically engaged in forming their identities while realigning their orientation away from adults and toward peers (Erikson, 1950, 1968; Larson \& Richards, 1991; Smetana et al., 2006). For Burmese refugee students and other ethnic minorities, finding one's ethnic identity is a part of this process (Marcia, 1966, 1980; Phinney, 1989). In diverse environments like the school where this study occurred, it is important that schools take a multicultural approach, which means they value the diverse traits students bring to school (Banks, 1993; Gay, 2004; Lee, 2003). 
Within the multicultural context where the study occurred, Burmese refugee students had many opportunities for intercultural interaction. Intercultural interaction can reduce prejudice, benefit students' identity formation, and help them learn norms to navigate the school system (Gurin et al., 2011; Ryan \& Patrick, 2001; Wentzel et al., 2012; Yoon, 2012). For refugee students, it is especially important that they learn the norms of the hegemonic culture because they face many challenges in their countries of resettlement (McBrien, 2005; Valenta, 2010). Helping refugee students achieve success often takes multiple forms of support (Block et al., 2014; Dávila, 2012; Kirova, 2012; McBrien \& Ford, 2012; Nwosu \& Barnes; 2014; Pugh et al., 2012; Taylor \& Sidhu, 2012). The experiences and needs of female refugee students has only been lightly studied (Harris, 2010; Oikonimody, 2007; 2009), and primarily with refugee students from Africa. The experiences of female Burmese refugee students in United States middle schools have not been studied.

To examine the experiences of Burmese refugee students, I chose three female participants to interview, observe, and conduct stimulated recall sessions. I employed a qualitative, transcendental phenomenological approach to emphasize the experiences of the participants (Moustakas, 1994). I used three forms of investigation to find confirming and disconfirming data so as to capture the true essence of the participants' experiences (Creswell, 2008, 2013).

I analyzed the data I collected by assigning codes according to a transcendental phenomenological approach in which I set aside my experiences while attempting to 
BURMESE REFUGEE STUDENT INTERACTION

merge the participants' experiences with their meanings of the experiences to arrive at an essence of their experiences (Moustakas, 1994).

\section{Synthesis of Findings}

Using interviews, observations, and stimulated recall, I investigated three research questions:

1. What were the experiences of Burmese refugees in multicultural middle school classrooms?

2. How did Burmese refugee students experience interactions with students from other cultural backgrounds?

3. How did the interactions Burmese refugee students have with students from other cultural backgrounds affect their school experiences, including learning and identity formation?

For the first research question (What were the experiences of Burmese refugees in a multicultural middle school classroom?), I found that the three female Burmese refugee students identified three major behaviors they employed to meet their ideals of being good students: (a) understanding what they were learning in class, (b) staying on task and being compliant, and (c) being kind to others and maintaining friendships. They viewed school as a place to learn, and though they may have had friends, they focused on learning and staying on task in their classrooms.

For the second research question (How did Burmese refugee students experience interactions with students from other cultural backgrounds?), I found that these three female Burmese refugee students acted out their conceptions of what a good student 
should be, regardless of the cultural background of their assigned partner. They consistently sought to understand the material they were supposed to be learning in class, stayed on task, and were kind to their partners.

For the third research question (How did the interactions Burmese refugee students have with students from other cultural backgrounds affect their school experiences, including learning and identity formation?), I found that the three Burmese refugee students acted out their ideas of what good students should be to solidify their identities as good students who focused on understanding, stayed on task, and were kind to others. They used their experiences in their countries of origin to frame their experiences in the United States. Because they acted out their conceptions of what a good student should be, they worked well with students from a variety of different cultural backgrounds. By working well with all other students, the participants reified their identities as good students.

The participants had strong notions of the behavioral norms they saw as helping students to be successful. They acted out those behavioral norms in their interactions with students from other cultural backgrounds, which, in turn, helped them view themselves as successful students. Though none of the participants would be classified as having an achieved ethnic identity (Marcia, 1966, 1980), all linked their school experiences in their countries of origin to successful behaviors in a multicultural middle school in the United States, leading to their identities as good students. 


\section{Situated in Larger Context}

The participants faced many of the same challenges refugees typically face (Dryden-Peterson, 2015; McBrien, 2005). Though I could not confirm whether or not any of the participants lived in poverty, they attended a school where approximately $90 \%$ of the students qualified for free or reduced-price lunch. Certainly, they were all unfamiliar with English when they first arrived in the United States, as evidenced by their inclusion in programs for English Language Learners. All three participants identified learning English as a struggle and shared anecdotes with me about experiences in their English language development classes.

Though many researchers have advocated holistic supports to help refugee students achieve success in their resettled homes (Block et al., 2014; McBrien \& Ford, 2012; Pugh et al., 2012; Taylor \& Sidhu, 2012), the only specific supports I examined were those provided in their school. As stated in Chapter 3, Ohma and Khalidah had previously been involved in a class for Burmese refugee students through the Schools

Uniting Neighborhoods program, but were not part of the group during the study (though Ohma was still involved in Schools Uniting Neighborhoods, she was not in the group for Burmese refugee students). Even if they had been involved with the Burmese group after school, there was little to no communication between the group and teachers at their school.

The clearest support offered to the participants during the school day was their inclusion in English language development classes. According to Dávila (2012), refugee students often equate learning English with success. Certainly, each of the three 
participants in this study placed a high priority on learning English and spoke of it as part of their successful approach to learning in general. The participants appreciated the supports offered by the English language program, as evidenced by both Ohma and Khalidah talking about events in their English language development classes in a positive way. Khalidah even credited her English language development teachers with helping her avoid confusion when she said, "Um, the teachers that I work with come pick me up because I didn't really know where to go," in response to a question about what she found confusing when she came to the United States. It seems that the students' experiences in their English language support classes were key in helping them achieve success.

At the same time they were becoming successful in school, all three participants continued to identify with their countries of origin. They appeared to take many of their views of what it meant to be a good student from their experiences in Muslim schools in their home countries. In other words, it seemed the participants' actions in schools in the United States were largely informed by what happened in their countries of origin. Their dual allegiances echoes the framework provided by Ogbu and Simons (1998) in describing refugees as having traits of both voluntary and involuntary immigrants. Simply put, voluntary immigrants chose to emigrate from their countries of origin and involuntary immigrants were conquered or enslaved by the country who took them in. Typically, according to Ogbu and Simons, voluntary immigrants adopt the norms of the host country whereas involuntary immigrants reject those norms. Refugees fall somewhere between. Because they were forced out of their countries of origin, they do 
not necessarily readily adopt the norms of the new country. However, because they were taken in by their new countries after being forced to leave their countries of origin, they do not necessarily reject the norms of the new country. Instead, they retain valued norms of their countries of origin while learning and adopting enough of the norms of their new countries to be successful.

My conclusions also echo a framework presented by Mosselson (2006), which focused on understanding refugee identity construction. Mosselson (2006) interviewed fifteen female Bosnian refugees and found they constructed their identities through, "roots \& routes" (p. 20), meaning they simultaneously looked back upon their origins and looked forward to their future. In this framework, refugee identities, "remained fluid" (p. 22). Instead of working toward a final stage of identity, the refugees in Mosselson's study constantly reshaped their identities.

In this study, Ohma, Khalidah, and Shamshidah held on to the norms they learned to be successful in their countries of origin and applied them in their new country. They valued understanding what they were learning, staying on task and following directions, and being kind to others. In embodying those traits in the classroom, they were able to successfully navigate classrooms in the United States. Next, I discuss how their successful navigation of classrooms in the United States affected the participants' identity formation.

According to French et al. (2006) and Gartner et al. (2014), intercultural interaction in multicultural contexts may be a prerequisite for ethnic minorities to effectively explore their ethnic identities (Marcia, 1980). Though the participants 
interacted equally well with students from a variety of different cultures, their interactions were very focused on the task at hand. Because their interactions were limited, they did not seem to be exploring their identities as students. Instead, they had settled on their identities as good students.

According to Marcia’s (1980) framework, Ohma, Khalidah, and Shamshidah would be in foreclosed statuses. They had settled on their identity as good students without actively exploring different options. However, outside of school, the participants had different orientations toward their friends. Ohma was friend-oriented; Khalidah was both friend-oriented and family-oriented; Shamshidah was family-oriented. According to Mosselson's (2006) framework, it makes sense that the participants were not settled in their identities, as refugee identities are always in flux. In this study, the only part of their identities that they had settled upon was good student. While other facets of their overall identity formation were still in process for each of the participants (Bucholtz and Hall, 2005; Esteban-Guitart \& Moll, 2014a; 2014b; Gee, 2000; Mosselson, 2006), in this study, I only examined their identities as students.

Because the participants were adolescents in middle school, they were certainly well into Erikson's $(1950,1968)$ identity versus role confusion stage. While forming their identities in this stage, young adolescents protect themselves against embarrassment (Erikson, 1950) and reorient themselves toward their peers (Larson \& Richards, 1991; Smetana et al., 2006). Ohma and Khalidah were more oriented toward peers than was Shamshidah, but Shamshidah still spoke of having several friends at school. This makes sense as they were each in the process of reorienting from family toward peers. Ohma 
was very friend-oriented; Khalidah was both friend- and family-oriented; Shamshidah was very family-oriented. I found it interesting that between the three participants, I saw a range of development along Erikson's $(1950,1968)$ identity versus role confusion stage.

Perhaps to help protect themselves against embarrassment, as Erikson (1950) asserted was a trait of his identity versus role confusion stage, the participants were reluctant to work with other students. Both Ohma and Shamshidah reported not wanting to work with other students. Khalidah reported wanting to work with small groups or partners, but typically worked alone before checking her answers with partners. Their reluctance to work with others could be related to their lack of experience with childcentered educational practices, making them uncomfortable when working with other students (Dryden-Peterson, 2015). Having been in the country for several years, the participants may have tempered their discomfort somewhat, but it seemed to still exist.

Despite their general reluctance to work with others, they worked equally well with students from a variety of cultural backgrounds, which helped solidify their identities as good students. Their ability to work equally well with students from different cultural backgrounds presents a different picture of intercultural interactions than one described by Halualani (2010). Halualani found that in a multicultural university, college students interacted with others differently depending on their ethnic backgrounds and the context in which the interactions occurred. According to Phinney et al. (2007), though, college students who have secure ethnic identities are more willing to engage with students from other ethnic identities than those who have not achieved 
secure identities. Phinney et al, however, did not report on whether those interactions were different depending on the ethnic backgrounds of those involved. It could be the case that as students explore their ethnic identities through intercultural interactions, they grow more willing to interact, but the interactions differentiate depending on the context and the people involved.

I contend that the context played a very large role in allowing the participants to learn and implement successful habitus. As stated earlier, in their middle school there were approximately 900 students who spoke over 35 different languages. Because of the super-diverse (Vertovec, 2007) context, there was no majority ethnic group. With no group strongly outnumbering other groups, the school did not have a singular set of accepted norms.

Bourdieu (1990) and Bourdieu and Wacquant (1992) described fields as places in which actors bring in their own dispositions, collective norms are created and reified by those whose habitus matches the accepted collective norms. According to Nolan (2012), the most valuable habitus become the norm. Those with more valuable habitus have more cultural capital, and, in turn, have more access to privilege. Though never directly stated by Bourdieu (1977, 1990), Boiurdieu and Wacquant (1992), or Nolan (2012), an underlying assumption of this framework is that there exists one group with privileged access to cultural capital; other groups are marginalized. In the context of this study, though, the assumption of one group having greater access to cultural capital did not seem to be the case. 
Due to the super-diverse context of the school (Vertovec, 2007), no groups of students were large enough to constitute anything resembling a hegemonic culture in their classes. Students fit into a wide variety of different groups (e.g. athletes, good students, trend-followers, goths) and cultures, but no groups dominated a classroom such that other groups of students were marginalized. In such a context, the participants did not have to learn to one particular set of norms; instead, they were able to be successful by acting out the values they brought from their countries of origin, or their own forms of capital (Yosso, 2005). In classroom contexts where all of their interactions were intercultural and there was no one set of norms they had to meet, they were able to adjust their behavior to work successfully with students from a variety of different cultural backgrounds. Their successful work with students from other cultural backgrounds in their classrooms, in turn, solidified their identities as good students.

Though I framed this study through the lens of theory of field and theory of social and cultural reproduction (Bourdieu, 1973, 1977, 1990; Bourdieu and Wacquant, 1992), and I believe it is a relevant lens to examine how Burmese refugee students experience their interactions with students from other cultural backgrounds, particularly in their learning and identity formation, it seems clear to me that in the multicultural middle school the participants attended, several sets of acceptable norms existed. Tzanakis (2011) suggested viewing schools as having one particular set of norms was dangerous in stating that schools do not blindly transit cultural capital; they are much more complex. Olneck (2000) examined how multicultural approaches to education, in which students' diverse backgrounds were valued, could affect cultural capital. In short, while 
multicultural education does not necessarily change existing cultural capital, it gives minority students a way to shape the formation of cultural capital, resulting in greater access to success.

In a study also framed through Bourdieu's (1977) theory of field, Dávila (2012) found that students had a difficult time navigating the classroom norms because they were not given ample opportunities to interact with other students and learn English sufficiently. Put in a multicultural context with increased opportunities for intercultural interaction, the students in Dávila's (2012) study may have been more successful. The participants in my study had ample opportunities for intercultural interaction. Those interactions helped reinforce the values they brought with them from their previous experiences and solidified their identities as good students.

The idea that a multicultural context, with opportunities for intercultural interaction, can help students become more successful in navigating school norms is certainly not new. Banki (2012) advocated intercultural interaction as a way for refugee students from Burma and Bhutan to feel more valued in the classroom, as well as help other students better understand the world. Kirova (2012) stated that multiple identities were able to exist and be valued simultaneously in multicultural environments.

Yosso (2005) emphasized that marginalized people bring their own capital into fields. With their own forms of capital, marginalized people could shape the norms of a field just as whose habitus have more value in the field. Because the field in this study was so diverse, the participants' habitus did not have to match one particular set of norms for them to be successful. Their habitus simply had to match a set of successful norms. 
For the participants in this study, they were able to be successful by acting out their values, brought from their countries of origin and shaped by their experiences in the United States, with students from a variety of different cultural backgrounds. In doing so, they worked toward solidifying their identities as good students.

The most important factor of the participants' solidifying their identities as good students (aside from the habitus of the participants themselves) was the super-diverse context (Vertovec, 2007) of the study. In less diverse contexts, the participants may not have been able to feel as comfortable in their intercultural interactions as they did. They all reported being reluctant to work with other students (though Khalidah less so than Ohma or Khalidah), which is a common challenge facing refugee students (DrydenPeterson, 2015), though they were able to engage in intercultural interactions very well. I largely attribute their willingness to engage in an activity despite their reluctance to their classrooms having no hegemonic group. The participants believed they could participate in class as well as anyone else, which opened them up to acting out their habitus, being successful, and reinforcing their identities as good students.

\section{Implications}

For Burmese refugee students to successfully navigate the challenges presented by their resettlement, especially in a multicultural middle school environment when they work to achieve secure ethnic identities, they need to be given opportunities to work with students from other cultural backgrounds. By working with students from other cultural backgrounds, Ohma, Khalidah, and Shamshidah were able to act out their values to work toward solidifying their identities as good students. Their focus on being good students 
allowed them to be successful among the various social norms created in the multicultural classrooms they attended.

Before discussing the implications of this study, I must begin with the caveat that the study was very narrowly focused on three particular Burmese refugee students in one multicultural middle school in the Pacific Northwest. Because I was teaching full time throughout the study, which lasted through the spring of 2015, I limited the interviews, observations, and stimulated recall sessions to my preparation period, before school, or after school. I focused my study on students' experiences in classrooms. Therefore, I had to gather all of my data before the school year ended. The conclusions are not meant to be widely generalizable. Instead, I hope to offer a glimpse of the experiences of some of the most marginalized students in a particular set of circumstances.

The implications from this study fall under two general categories: practice and research. Based on the conclusions from this study, I suggest teachers set up opportunities for intercultural interaction, vary the arrangements for intercultural interactions, and emphasize multiculturalism in their classrooms. I also recommend that researchers continue to explore the experiences of marginalized students, especially refugee students. They need to continue to examine how classroom contexts affect the experiences of marginalized students. Last, they need to consider identity as both everchanging and multi-faceted.

\section{Implications for Practice}

The overriding goal of my study was to explore the experiences Burmese refugee students had in a multicultural middle school setting where I was also a teacher. I aimed 
to understand their experiences so I could help design better learning experiences for students who have been traditionally marginalized. Though my study was very specific, it was rooted in the desire to improve the educational experiences of some of the most marginalized students in the United States.

None of the participants' teachers intentionally paired them with students from other cultural backgrounds; the teachers simply paired students without considering their respective ethnic backgrounds. In the multicultural classrooms they attended, every interaction I observed was intercultural. The participants (especially Ohma and Shamshidah) in this study reluctantly engaged in those interactions, but reported they would generally rather not work with others if given the choice. Despite their reluctance, the interactions helped the students act out their ideas about the nature of good students. Having the opportunities to act out their ideas of being good students allowed them to integrate their beliefs about school they developed in their countries of origin with their current identities in the United States. An integral part of the participants' success in class was the multicultural context in which they worked with other students.

In classrooms that are not as multicultural as those in the study, it would be important for teachers to intentionally set up intercultural interactions. Those intercultural interactions may help marginalized students navigate the various norms existing in a classroom (Gurin et al., 2011; Ryan \& Patrick, 2001; Wentzel et al., 2012; Yoon, 2012). In my study, the participants used the intercultural interactions to work toward solidifying their identities as good students. Other students may react differently, 
but giving them opportunities to engage in intercultural interactions seems to be beneficial.

I recommend teachers vary the students with whom refugee students work when they set up intercultural interactions. Ohma, Khalidah, and Shamshidah did not report any preference for their work partners and appeared to work equally well with everyone I observed. I attribute their flexibility to the super-diverse context (Vertovec, 2007) in their middle school. In all, the participants worked with students from at least seven different cultural backgrounds during observation sessions alone. In less diverse contexts, students might express a preference for certain groups of students, possibly limiting their exploration and inhibiting their ethnic identity formation. Educators in contexts with less diversity would need to ensure the widest variety of groupings possible by being intentional about varying with whom refugee students work.

In addition to varying the work partners and groups, I suggest intercultural interactions be built around a culture of multiculturalism in the classroom. In my study, the context was super-diverse (Vertovec, 2007), so emphasizing multiculturalism was not a stretch for teachers. In less diverse settings, it may be more important for teachers to intentionally emphasize multiculturalism. Not only do multicultural approaches appear to benefit minority students academically (Lee, 2003; Malsbary, 2014; Martinez, 2010; Paris, 2009), they may help minority students gain power by allowing for multiple sets of norms to exist, thereby reducing marginalization (Olneck, 2000). In the multicultural context of my study, the availability of multiple sets of norms with which the students could be successful helped Burmese refugee students be successful in the classroom and 
affirmed their identities as good students. In less multicultural contexts, teachers may have to intentionally stress multiculturalism for marginalized students to affect the classroom habitus and gain cultural capital.

For classroom practice to be most beneficial for marginalized students, it must be based on solid research. Next I discuss my recommendations for research moving forward from my dissertation study.

\section{Implications for Research}

My study explored the experiences of a small number of students from a relatively small population in a particular setting. The intent was not to make conclusions that could be widely generalizable, but rather to provide an in-depth picture of the experiences of one specific group of students. The implications for future research are three-fold: (a) continue to explore the experiences of marginalized students, especially refugee students, (b) focus on different contexts, and (c) treat identity as ever changing and multi-faceted.

Though my sample size was small, I was able to examine the experiences of three students from a refugee population — one with scant research to date. To provide high quality education to all students, including refugee and other marginalized students, educators need to understand their experiences. For this reason, I recommend that researchers continue studying the experiences of individual groups of marginalized students (Portes \& Zhou, 1993). McBrien (2005) and Portes and Zhou (1993) advocated differentiating the experiences of different refugee populations. To differentiate those experiences, researchers would need to have a deep understanding of the experiences of 
various refugee populations. To develop a deep understanding of different refugee populations, more research on various groups is needed. More research on the experiences of different groups of students would lead to better classroom practices to support them.

One of the most important findings in my study was the importance of the classroom context to the participants' learning and identity formation. Specifically, the multicultural context allowed for multiple sets of norms to exist in which the participants could match their habitus. Different contexts may have different effects on the experiences of marginalized students. When researchers examine the experiences of various students in classes, I suggest they pay careful attention to the context. In my study, I wondered how different the participants' experiences would have been if they attended a middle school with less diversity. Would the norms in those classrooms have been different than those in multicultural classrooms? Would they have been able to navigate the norms if the norms had been different? Would they have had sufficient opportunities for intercultural interaction? What would their identity formation process have looked like?

Based on the conclusions in this study, I recommend researchers examine identity formation, especially for ethnic minorities, as a multi-faceted process. The participants in my study had all settled on identities as good students in school. Outside of school, however, they were in different stages in their identity formation processes according to Erikson's (1950) framework. If they were foreclosed according to Marcia's (1980) framework, but still in process of shifting their orientations from parents to peers 
(Smetana et al., 2006), it follows that adolescents have multiple identities continually in process. According to Mosselson (2006), refugee students' identity formation is in a constant state of flux. If the identity formation process for refugees is constantly changing, it makes sense that it may happen in more than one area.

As researchers continue to examine the experiences of various groups of students, we can continue to grow our understanding of the variety of experiences students have in various classrooms. Understanding the experiences of various students could allow teachers and researchers to design better learning experiences in their classrooms.

\section{Summary and Conclusion}

After exploring the experiences of three female Burmese refugee students in a multicultural middle school, especially focusing on their intercultural interactions, it is clear to me that more work needs to be done to improve educational access for marginalized students. I found the participants to highly value trying to understand what they were learning, staying on task, and being kind to other students. In doing these three things, they solidified their identities as good students. They acted out what they knew and valued from their past experiences while working hard to make better futures for themselves.

Because of the super-diverse context (Vertovec, 2007) of their classrooms, a piece of the participants' work toward identifying as good students was interacting with students from other cultural backgrounds. Even though the participants were generally reluctant to work with other students, they worked equally well with students from various cultural backgrounds. They understood that working with others helps them 
better understand the material they were supposed to learn. Intercultural interactions also helped the participants navigate several different norms to be successful. Their habitus seemed to match at least one of many sets of successful norms.

Moving forward, for refugee students to be successful in classrooms in the United States, I suggest teachers intentionally set up intercultural interactions in which they vary the groupings. I also suggest teachers set up these intercultural interactions in the context of a culture of multiculturalism. When it comes to research, I suggest more studies intended to deeply understand the experiences of a variety of marginalized students. I also suggest researchers consider the contexts when studying the experiences of marginalized students. Another recommendation I have is to further explore identity development as a multifaceted process.

In the end, the three participants in this study, Ohma, Khalidah, and Shamshidah, had different, but similar, experiences in their multicultural middle school. They wanted to be good students, and used their values of seeking understanding, staying on task, and being kind to others to solidify their identities as good students. Intercultural interactions helped them solidify those identities as well as navigate various norms in their superdiverse context. I fully expect each participant to continue to work hard and become more and more successful as they move forward in school. As they increase their English language ability and learn more ways to navigate the various norms in their schools, I expect them each to increase their capacities to find ways to be successful. 


\section{References}

Albert, M., \& Kleinman, D. L. (2011). Bringing Pierre Bourdieu to science and technology studies. Minerva, 49(3), 263-273. doi: 10.1007/s11024-011-9174-2

Allport, G. W. (1954). The nature of prejudice. Cambridge, MA: Addison Wesley.

Alfassi, M. (2009). The efficacy of a dialogic learning environment in fostering literacy. Reading Psychology, 30(6), 539-563. doi: 10.1080/02702710902733626

Alitolppa-Niitamo, A. (2004). Somali youth in the context of schooling in metropolitan Helsinki: A framework for assessing variability in educational performance. Journal of Ethnic and Migration Studies, 30(1), 81-106. doi: $10.1080 / 1369183032000170187$

Ata, A., Bastian, B., \& Lusher, D. (2009). Intergroup contact in context: The mediating role of social norms and group-based perceptions on the contact-prejudice link. International Journal of Intercultural Relations, 33(6), 498-506. doi:10.1016/j.ijintrel.2009.05.001

Bailey, K. D. (1994). Methods of social research (4th ed.). New York, NY: The Free Press.

Banki, S. (2012). Refugees as educators: The potential for positive impact on educational systems. In F. McCarthy \& M. Vickers (Eds.), Refugee and immigrant students: Achieving equity in education (pp. 43-64). Charlotte, NC: Information Age.

Banks, J. A. (1993). Multicultural education: Historical development, dimensions, and practice. Review of Research in Education, 19(1), 3-49. 
Banks, C. A. M., \& Banks, J. A. (1995). Equity pedagogy: An essential component of multicultural education. Theory into Practice, 34(3), 152-158.

Banks, J. A. (2008). Diversity, group identity, and citizenship education in a global age. Educational Researcher, 37(3), 129-139.

Barriball, K. L., \& While, A. (1994). Collecting data using a semi-structured interview: A discussion paper. Journal of Advanced Nursing, 19(2), 328-335.

Bastian, B., Lusher, D., \& Ata, A. (2012). Contact, evaluation and social distance: Differentiating majority and minority effects. International Journal of Intercultural Relations, 36(1), 100-107. doi:10.1016/j.ijintrel.2011.02.005

Bennett, C. (2001). Genres of research in multicultural education. Review of Educational Research, 71(2), 171-217.

Berndt, T. J. (1979). Developmental changes in conformity to peers and parents. Developmental Psychology, 15(6), 608-616. doi:10.1037/0012-1649.15.6.608

Binder, J., Zagefka, H., Brown, R., Funke, F., Kessler, T., Mummendey, A.,...Leyens, J. P. (2009). Does contact reduce prejudice or does prejudice reduce contact? A longitudinal test of the contact hypothesis among majority and minority groups in three European countries. Journal of Personality and Social Psychology, 96(4), 843-856. doi:10.1037/a0013470

Bird, J. N., Brough, M. K., \& Cox, L. (2012). Producing solidarity: A settlement strategy of the Karen refugee community in Brisbane, Australia. In M. Nocker \& G. Pearson (Eds.), Ethnographic horizons in times of turbulence (pp. 1-21). 
BURMESE REFUGEE STUDENT INTERACTION

Unpublished manuscript, University of Liverpool Management School, Liverpool, England.

Block, K., Cross, S., Riggs, E., \& Gibbs, L. (2014). Supporting schools to create an inclusive environment for refugee students. International Journal of Inclusive Education, 18(12), 1337-1355. doi:10.1080/13603116.2014.899636

Bloom, B. S. (1953). Thought-processes in lectures and discussions. The Journal of General Education, 7(3), 160-169.

Bourdieu, P. (1973). Cultural reproduction and social reproduction. In R. Brown (Ed.), Knowledge, education and cultural change (pp. 71-112). London, United Kingdom: Tavistock.

Bourdieu, P. (1977). Outline of a theory of practice (R. Nice, Trans.). Cambridge, United Kingdom: Cambridge University Press.

Bourdieu, P. (1990). In other words: Essays towards a reflexive sociology (M. Adamson, Trans). Stanford, CA: Stanford University Press.

Bourdieu, P., \& Wacquant, L. J. D. (1992). An invitation to reflexive sociology. Chicago, IL: The University of Chicago Press.

Bowerman, C. E., \& Kinch, J. W. (1959). Changes in family and peer orientation of children between the fourth and tenth grades. Social Forces, 37(3), 206-211. doi: $10.2307 / 2572963$

Bucholtz, M., \& Hall, K. (2005). Identity and interaction: A sociocultural linguistic approach. Discourse Studies, 7(4-5), 585-614. doi: 10.1177/1461445605054407.

Calderhead, J. (1981). Stimulated recall: A method for research on teaching. British 
BURMESE REFUGEE STUDENT INTERACTION

Journal of Educational Psychology, 51(2), 211-217.

Caskey, M. M., \& Anfara, V. A., Jr. (2014). Developmental characteristics of young adolescents: Research summary. Association for Middle Level Education. Retrieved from https://www.amle.org/BrowsebyTopic/WhatsNew/WNDet/TabId/270/ArtMID/88 8/ArticleID/455/Developmental-Characteristics-of-Young-Adolescents.aspx

Chan, E. (2007). Student experiences of a culturally sensitive curriculum: Ethnic identity development amid conflicting stories to live by. Journal of Curriculum Studies, 39(2), 177-194. doi: 10.1080/00220270600968658

Cheah, W. H., Karamehic-Muratovic, A., Matsuo, H., \& Poljarevic, A. (2011). The role of language competence, interpersonal relationships, and media use in Bosnian refugees' resettlement process. Journal of Intercultural Communication Research, 40(3), 219-236. doi: 10.1080.17475759.2011.618842

Cole, M. (2009). On 'white supremacy' and caricaturing, misrepresenting and dismissing Marx and Marxism: A response to David Gillborn's “Who’s afraid of critical race heory in education." Journal for Critical Education Policy, 7(1), 2949.

Cole, M., \& Maisuria, A. (2007). 'Shut the f*** up,' 'you have no rights here:' Critical race theory and racialization in post-7/7 racist Britain. Journal for Critical Education Policy Studies, 5(1), 94-120. 
Cotton, D. R. E., George, R., \& Joyner, M. (2013). Interaction and influence in culturally mixed groups. Innovations in Education and Teaching International, 50(3), 272-283. doi: 10.1080/14703297.2012.760773

Creswell, J. W. (2008). Educational research: Planning, conducting, and evaluating quantitative and qualitative research ( $3^{\text {rd }}$ ed.). Upper Saddle River, NJ: Pearson.

Creswell, J. W. (2009). Research design: Qualitative, quantitative, and mixed methods approaches $\left(3^{\text {rd }}\right.$ ed. $)$. Thousand Oaks, CA: Sage.

Creswell, J. W. (2013). Qualitative inquiry \& research design: Choosing among five approaches $\left(3^{\text {rd }}\right.$ ed.). Thousand Oaks, CA: Sage.

Cronbach, L. J. (1975). Beyond the two disciplines of scientific psychology. American Psychologist, 30(2), 116-127.

Cross, E. B. (1854). On the Karens. Journal of the American Oriental Society, 4(298), 291-316.

Daoud, A. M. (2003). “The ESL kids are over there": Opportunities for social interactions between immigrant Latino and white high school students. Journal of Hispanic Higher Education, 2(3), 292-314. doi:10.1177/1538192703002003006

Dávila, L. T. (2012). 'For them it's sink or swim': Refugee students and the dynamics of migration, and (dis)placement in school. Power and Education, 4(2), 139-149.

deAnda, D. (1984). Bicultural socialization: Factors affecting the minority experience. Social Work, 29(2), 101-107. 
den Brok, P., Fisher, D., Rickards, T., \& Bull, E. (2006). Californian science students' perceptions of their classroom learning environments. Educational Research \& Evaluation, 12(1), 3-25.

Denzin, N. K. (1989). The research act: A theoretical introduction to sociological methods $\left(3^{\text {rd }}\right.$ ed.). Englewood Cliffs, NJ: Prentice Hall.

DeWitt, J., \& Osborne, J. (2010). Recollections of exhibits: Stimulated-recall interviews with primary school children about science centre visits. International Journal of Science Education, 32(10), 1365-1388. doi: 10.1080/09500690903085664

Dooley, K. (2012). Positioning refugee students as intellectual class members. In F. McCarthy \& M. Vickers (Eds.), Refugee and immigrant students: Achieving equity in education (pp. 3-20). Charlotte, NC: Information Age.

Dryden-Peterson, S. (2015). The educational experiences of refugee children in countries of first asylum. Retrieved from the Migration Policy Institute website: http://www.migrationpolicy.org/research/educational-experiences-refugeechildren-countries-first-asylum

Erikson, E. (1950). Childhood and society. New York, NY: W. W. Norton \& Company. Erikson, E. (1968). Identity: Youth and crisis. New York, NY: W. W. Norton \& Company.

Esteban-Guitart, M., \& Moll, L. C. (2014a). Funds of identity: A new concept based on the funds of knowledge approach. Culture \& Psychology, 20(1), 31-48. doi: $10.1177 / 1354067 X 13515934$ 
Esteban-Guitart, M., \& Moll, L. C. (2014b). Lived experience, funds of identity, and education. Culture \& Psychology, 20(1), 70-81. doi:

$10.1177 / 1354067 X 13515940$

Ferfolja, T., \& Vickers, M. (2010). Supporting refugee students in school education in Greater Western Sydney. Critical Studies in Education, 51(2), 149-162. doi: $10.1080 / 17508481003731034$

French, S. E., Seidman, E., Allen, L., \& Aber, J. L. (2006). The development of ethnic identity during adolescence. Developmental Psychology, 42(1), 1-10. doi: $10.1037 / 0012-1649.42 .1 .1$

Fuertes, A. (2010). Birds inside a cage: Metaphor for Karen refugees. Social Alternatives, 29(1), 20-24.

Gaier, E. L. (1954). Memory under conditions of stimulated recall. Journal of General Psychology, 50(1), 147-153.

Gartner, M., Kiang, L., \& Supple, A. (2014). Prospective links between ethnic socialization, ethic and American identity, and well-being among Asian-American adolescents. Journal of Youth and Adolescence, 43(10), 1715-1727. doi: 10.1007/s10964-013-0044-0

Gass, S. M., \& Mackey, A. (2000). Stimulated recall methodology in second language research. Mahwah, NJ: Lawrence Erlbaum.

Gay, G. (2004). Curriculum theory and multicultural education. In J. A. Banks \& C. A. McGee Banks (Eds.), Handbook of research on multicultural education (pp. 3049). San Francisco, CA: Jossey-Bass. 
Gay, G. (2009). Similar concerns, different perspectives of social studies and multicultural education. Social Studies Review, 48(1), 25-27.

Gee, J. P. (2000). Identity as an analytic lens for research in education. Review of Research in Education, 25, 99-125.

Gersten, R., \& Baker, S. (2000). What we know about effective instructional practices for English-language learners. Exceptional Children, 66(4), 454-470.

Gilhooly, D., \& Lee, E. (2014). The role of digital literacy practices on refugee resettlement: The case of three Karen brothers. Journal of Adolescent and Adult Literacy, 57(5), 387-396. doi: 10.1002/JAAL.254

Gillborn, D. (2010). Full of sound and fury, signifying nothing? A reply to Dave Hill's "Race and class in Britain: A critique of the statistical basis for critical race theory in Britain.” Journal for Critical Education Policy Studies, 8(1), 78-107.

Gitlin, A., Buendía, E., Crosland, K., \& Doumbia, F. (2003). The production of margin and center: Welcoming-unwelcoming of immigrant students. American Educational Research Journal, 40(1), 91-122. doi: 10.3102/00028312040001091

Greenspan, I. (2014). How can Bourdieu's theory of capital benefit the understanding of advocacy NGOs? Theoretical framework and empirical illustration. Nonprofit and Voluntary Sector Quarterly, 43(1), 99-120. doi: 10.1177/0899764013501580.

Guba, E. G., \& Lincoln, Y. A. (2005). Paradigmatic controversies, contradictions, and emerging confluences. In N. K. Denzin \& Y. S. Lincoln (Eds.), The Sage 
BURMESE REFUGEE STUDENT INTERACTION

handbook of qualitative research $\left(3^{\text {rd }}\right.$ ed.) (pp. 191-215). Thousand Oaks, CA: Sage.

Gurin, P., Nagda, B. A., \& Sorensen, N. (2011). Intergroup dialogue: Education for a broad conception of civic engagement. Liberal Education, 97(2), 46-51.

Gudykunst, W. B. (Ed.). (2005). Theorizing about intercultural communication. Thousand Oaks, CA: Sage.

Habermas, T., \& Bluck, S. (2000). Getting a life: The emergence of the life story in adolescence. Psychological Bulletin, 126(5), 748-769.

Halualani, R. T. (2010). Interactant-based definitions of intercultural interaction at a multicultural university. The Howard Journal of Communications, 21(3), 247272. doi: $10.1080 / 10646175.2010 .496666$

Halualani, R. T., Chitgopekar, A., Huynh, J., Morrison, T. A., \& Dodge, P. S. W. (2004). Who's interacting? And what are they talking about?-Intercultural contact and interaction among multicultural university students. International Journal of Intercultural Relations, 28(5), 353-372. doi: 10.1016/j.ijintrel.2004.08.004

Harris, A. (2010). I ain't no girl: Representation and reconstruction of the 'found girls' of Sudan. Race/Ethnicity: Multidisciplinary Global Contexts, 4(1), 41-63.

Hosseini-Maasoum, S. M. (2012). The importance of learner-learner and learnerinstructor interaction among EFL learners in Payame Noor University: A constructivist approach. Journal of Educational and Social Research, 2(2), 201208. doi: 10.5901/jesr.2012.v2n2.201 
Hviid, P., \& Villadsen, J. W. (2014). Cultural identities and their relevance to school practice. Culture \& Psychology, 20(1), 59-69. doi: 10.1177/1354067X13515938

Isik-Ercan, Z. (2012). In pursuit of a new perspective in the education of children of the refugees: Advocacy for the "family." Educational Sciences: Theory \& Practice, 12(4), 3025-3038.

Janzen, J. (2008). Teaching English language learners in the content areas. Review of Educational Research, 78(4), 1010-1038. doi: 10.3102/0034654308325580

Kagan, N., Krathwohl, D. R., \& Miller, R. (1963). Stimulated recall in therapy using video tape: A case study. Journal of Counseling Psychology, 10(3), 237-243.

Kiefer, S. M., Matthews, Y. T., Montesino, M., Arango, L., \& Preece, K. K. (2013). The effects of contextual and personal factors on young adolescents' social goals. The Journal of Experimental Education, 81(1), 44-67. doi:

$10.1080 / 00220973.2011 .630046$

Kiragu, E., Li Rosi, A., \& Morris, T. (2011). States of denial: A review of UNHCR's response to the protracted situation of stateless Rohingya refugees in Bangladesh. Policy Development and Evaluation Service, UNHCR. Retrieved from: http://www.unhcr.org/4ee754c19.pdf.

Kirova, A. (2012). Creating shared learning spaces: An intercultural, multilingual early learning program for preschool children from refugee families. In F. McCarthy \& M. Vickers (Eds.), Refugee and immigrant students: Achieving equity in education (pp. 21-42). Charlotte, NC: Information Age. 
Kovacev, L., \& Shute, R. (2004). Acculturation and social support in relation to psychosocial adjustment of adolescent refugees resettled in Australia. International Journal of Behavioral Development, 28(3), 259-267. doi: $10.1080 / 01650250344000497$

Krathwohl, D. R. (2009). Methods of educational and social science research: The logic of methods $\left(3^{\text {rd }}\right.$ ed.). Lake Grove, IL: Waveland Press.

Ladson-Billings, G. (2009). Just what is critical race theory and what's it doing in a nice field like education? In E. Taylor, D. Gillborn, \& G. Ladson-Billings (Eds.), Foundations of critical race theory in education (pp. 17-36). New York, NY: Routledge.

Larson, R., \& Richards, M. H. (1991). Daily companionship in late childhood and early adolescence: Changing developmental contexts. Child Development, 62(2), 284300. doi:10.2307/1131003

Lee, B. K., \& Chen, L. (2000). Cultural communication competence and psychological adjustment: A study of Chinese immigrant children's cross-cultural adaptation in Canada. Communication Research, 27(6), 764-792. doi:

$10.1177 / 009365000027006004$

Lee, I., \& Koro-Ljungberg, M. (2007). A phenomenological study of Korean students' acculturation in middle schools in the USA. Journal of Research in International Education, 6(1), 95-117.

Lee, O. (2003). Equity for linguistically and culturally diverse students in science education: A research agenda. Teachers College Record, 105(3), 465-489. 
BURMESE REFUGEE STUDENT INTERACTION

Leonardo, Z. (2004). The unhappy marriage between Marxism and race critique:

Political economy and the production of racialized knowledge. Policy Futures in Education, 2(3 \& 4), 483-493.

Leonardo, Z. (2009). The color of supremacy: Beyond the discourse of "white privilege.” In E. Taylor, D. Gillborn, \& G. Ladson-Billings (Eds.), Foundations of critical race theory in education (pp. 261-276). New York, NY: Routledge.

Letchamanan, H. (2013). Myanmar's Rohingya refugees in Malaysia: Education and the way forward. Journal of International and Comparative Education, 2(2), 86-97.

Leung, A. K., \& Chiu, C. (2010). Multicultural experience, idea receptiveness, and creativity. Journal of Cross-Cultural Psychology, 41(5-6), 723-741. doi:10.1177/0022022110361707

Lincoln, Y. S., \& Guba, E. G. (1985). Naturalistic inquiry. Beverly Hills, CA: Sage. Lyle, J. (2003). Stimulated recall: A report on its use in naturalistic research. British Educational Research Journal, 29(6), 861-878. doi: $10.1080 / 014119203200137349$

Malsbary, C. B. (2014). "It's not just learning English, it's learning other cultures": Belonging, power, and possibility in an immigrant contact zone. International Journal of Qualitative Studies in Education, 27(10), 1312-1336. doi: $10.1080 / 09518398.2013 .837210$

Marcia, J. E. (1966). Development and validation of ego-identity status. Journal of Personality and Social Psychology, 3(5), 551-558. 
Marcia, J. E. (1980). Identity in adolescence. In J. Adelson (Ed.), Handbook of adolescent psychology (pp. 159-187). New York, NY: Wiley.

Marshall, S. L., \& While, A. E. (1994). Interviewing respondents who have English as a second language: Challenges encountered and suggestions for other researchers. Journal of Advanced Nursing, 19(3), 566-571.

Martin, D. C., \& Yankay, J. E. (2012). Refugees and asylees: 2011. Retrieved from Department of Homeland Security, Office of Immigration Statistics website: http://www.dhs.gov

Martínez, R. A. (2010). "Spanglish" as a literacy tool: Toward an understanding of the potential role of Spanish-English code-switching in the development of academic literacy. Research in the Teaching of English, 45(2), 124-149.

Maxwell, J. A. (2013). Qualitative research design: An interactive approach ( $\left.3^{\mathrm{rd}} \mathrm{ed}.\right)$. Thousand Oaks, CA: Sage.

McAdams, D. P. (2001). The psychology of life stories. Review of General Psychology, $5(2), 100-122$.

McBrien, J. L. (2005). Educational needs and barriers for refugee students in the United States: A review of the literature. Review of Educational Research, 75(3), 329364.

McBrien, J. L., \& Ford, J. (2012). Serving the needs of refugee children and families through a culturally appropriate liaison service. In F. McCarthy \& M. Vickers (Eds.), Refugee and immigrant students: Achieving equity in education (pp. 107126). Charlotte, NC: Information Age. 
McCarthy, F. E., \& Vickers, M. H. (2012). Education for immigrants and refugees:

Creating equitable pathways for the future. In F. McCarthy \& M. Vickers (Eds.), Refugee and immigrant students: Achieving equity in education (pp. xiii-xxv). Charlotte, NC: Information Age.

McIntosh, P. (1988). White privilege: Unpacking the invisible knapsack. In P. S. Rothenberg (Ed.), Race, class, and gender in the United States: An integrated study (4 $4^{\text {th }}$ ed., pp. 165-169). New York, NY: St Martin's Press.

McLaren, P. (2009). Critical pedagogy: A look at the major concepts. In A. Darder, M. P. Baltodano, \& R. D. Torres (Eds.), The critical pedagogy reader (pp. 61-83). New York, NY: Routledge.

McLean, K. C., Breen, A. V., \& Fournier, M. A. (2010). Constructing the self in early, middle, and late adolescent boys: Narrative identity, individuation, and wellbeing. Journal of Research on Adolescence, 20(1), 166-187.

Morgan, A. (2007). Using video-stimulated recall to understand children's perceptions of learning in classroom settings. European Early Childhood Education Research Journal, 15(2), 213-226. doi: 10.1080/13502930701320933

Mosselson, J. (2006). Roots and roots: A re-imagining of refugee identity constructions and the implications for schooling. Current Issues in Comparative Education, $9(1), 20-29$.

Moustakas, C. (1994). Phenomenological research methods. Thousand Oaks, CA: Sage.

Nilsen, M. (2013). Will democracy bring peace to Myanmar? International Area 
Studies Review, 16(2), 115-141. doi: 10.1177/2233865913492961

Nolan, K. (2012). Dispositions in the field: Viewing mathematics teacher education through the lens of Bourdieu's social field theory. Educational Studies in Mathematics, 80(1-2), 201-215. doi: 10.1007/s10649-011-9355-9

Nwosu, O. C., \& Barnes, S. L. (2014). Where 'Difference is the norm': Exploring refugee student ethnic identity development, acculturation, and agency at Shaw Academy. Journal of Refugee Studies, 27(3), 434-456. doi: 10.1093/jrs/fet050

O’Brien, J. (1993). Action research through stimulated recall. Research in Science Education, 23(1), 214-221.

Ogbu, J. U. (2004). Collective identity and the burden of "acting white" in Black history, community, and education. The Urban Review, 36(1), 1-35.

Ogbu, J. U., \& Simons, H. D. (1998). Voluntary and involuntary minorities: A culturalecological theory of school performance with some implications for education. Anthropology \& Education Quarterly, 29(2), 155-188.

Oikonomidoy, E. (2007). 'I see myself as a different person who [has] acquired a lot...': Somali female students' journeys to belonging. Intercultural Education, 18(1), 15-27. doi: 10.1080/14675980601143611

Oikonomidoy, E. (2009). The multilayered character of newcomers' academic identities: Somali female high-school students in a US school. Globalisation, Societies and Education, 7(1), 23-39. doi: 10.1080/14767720802677358

Oh, S. A. (2012). Identity and inclusion: Education in refugee camps in Thailand. In F. 
BURMESE REFUGEE STUDENT INTERACTION

McCarthy \& M. Vickers (Eds.), Refugee and immigrant students: Achieving equity in education (pp. 65-85). Charlotte, NC: Information Age.

Oh, S. A., \& van der Stowe, M. (2008). Education, diversity, and inclusion in Burmese refugee camps in Thailand. Comparative Education Review, 52(4), 589-617.

Olneck, M. (2000). Can multicultural education change what counts as cultural capital? American Educational Research Journal, 37(2), 317-348. doi: $10.3102 / 00028312037002317$

Oregon Department of Human Services. (n.d.). Data: Refugees in Oregon. Retrieved from http://www.oregon.gov/dhs/assistance/pages/refugee/data.aspx

Para, E. A., (2008). The role of social support in identity formation: A literature review. Graduate Journal of Counseling Psychology, 1(1), Article 9.

Paris, D. (2009). "They're in my culture, they speak the same way": African American language in multiethnic high schools. Harvard Educational Review, 79(3), 428447.

Peters, G. (2014). Explanation, understanding and determinism in Pierre Bourdieu's sociology. History of the Human Sciences, 27(1), 124-149. doi: $10.1177 / 0952695113500974$

Pettigrew, T. F., Christ, O., Wagner, U., \& Stellmacher, J. (2007). Direct and indirect intergroup contact effects on prejudice: A normative interpretation. International Journal of Intercultural Relations, 31(4), 411-425

doi:10.1016/j.ijintrel.2006.11.003 
Pettigrew, T. F., \& Tropp, L. R. (2006). A meta-analytic test of intergroup contact theory. Journal of Personality and Social Psychology, 90(5), 751-783. doi:10.1037/0022-3514.90.5.751

Pettigrew, T. F., \& Tropp, L. R. (2008). How does intergroup contact reduce prejudice? Meta-analytic tests of three mediators. European Journal of Social Psychology, 38(6), 922-934. doi:10.1002/ejsp.504

Phinney, J. S. (1988, June). The development of ethnic identity in adolescents. Paper presented at the Utah State University Workshop on Identity Formation: Theoretical and Empirical Issues, Logan, UT.

Phinney, J. S. (1989). Stages of ethnic identity development in minority group adolescents. The Journal of Early Adolescence, 9(1-2), 34-49.

Phinney, J. S., Jacoby, B., \& Silva, C. (2007). Positive intergroup attitudes: The role of ethnic identity. International Journal of Behavioral Development, 31(5), 478490. doi: $10.1177 / 0165025407081466$

Piccolo, D. L., Harbaugh, A. P., Carter, T. A., Capraro, M. M., \& Capraro, R. M. (2008). Quality of instruction: Examining discourse in middle school mathematics instruction. Journal of Advanced Academics, 19(3), 376-410.

Piller, I. (2011). Intercultural communication: A critical introduction. Edinburgh, Scotland: Edinburgh University Press.

Pinson, H., \& Arnot, M. (2007). Sociology of education and the wasteland of refugee education research. British Journal of Sociology of Education, 28(3), 399-407. doi: 10.1080/01425690701253612 
Plano Clark, V. L., \& Creswell, J. W. (2010). Understanding research: A consumer's guide. Boston, MA: Merrill.

Polio, C., Gass, S., \& Chapin, L. (2006). Using stimulated recall to investigate native speaker perceptions in native-nonnative speaker interaction. Studies in Second Language Acquisition, 28(2), 237-267. doi: 10.1017/S0272263106060116

Portes, A., \& Zhou, M. (1993). The new second generation: Segmented assimilation and its variants. Annals of the American Academy of Political and Social Science, $530,74-96$.

Pugh, K., Every, D., \& Hattam, R. (2012). Inclusive education for students with refugee experience: Whole school reform in a South Australian primary school. The Australian Educational Researcher, 39, 125-141.

Purkey, M. (2006). A survival story from the Thai-Burmese border: The struggle for education in the Burmese community in Thailand. Refugee: Canada's Journal on Refugees, 23(1), 40-44.

Ragland, T. K. (1994). Burma's Rohingyas in crisis: Protection of "humanitarian" refugees under international law. Boston College Third World Law Journal, $14(2), 301-336$.

Rahman, U. (2010). The Rohingya refugee: A security dilemma for Bangladesh. Journal of Immigrant \& Refugee Studies, 8(2), 233-239. doi: $10.1080 / 15562941003792135$ 
Ranard, D. A., \& Barron, S. (Eds). (2007). Refugees from Burma: Their backgrounds and refugee experiences. Washington, DC: Center for Applied Linguistics, Cultural Orientation Resource Center.

Rosbrook, B., \& Schweitzer, R. D. (2010). The meaning of home for Karen and Chin refugees from Burma: An interpretative phenomenological approach. European Journal of Psychotherapy and Counseling, 12(2), 159-172.

Rubin, H. J., \& Rubin, I. S. (2005). Qualitative interviewing: The art of hearing data ( $2^{\text {nd }}$ ed.). Thousand Oaks, CA: Sage.

Ryan, A. M., \& Patrick, H. (2001). The classroom social environment and changes in adolescents' motivation and engagement during middle school. American Educational Research Journal, 38(2), 437-460.

Salazar, P. (2012). Exploring learners' noticing of corrective feedback through stimulated recall. Utrecht Studies in Language \& Communication, 24, 279-293.

Seidman, I. (2013). Interviewing as qualitative research: A guide for researchers in education $\&$ the social sciences $\left(4^{\text {th }}\right.$ ed.). New York, NY: Teachers College Press.

Sfard, A., \& Prusak, A. (2005). Telling identities: In search of an analytic tool for investigating learning as a culturally shaped activity. Educational Researcher, 34(4), 14-22. doi: 10.3102/0013189X034004014

Sleeter, C. E., \& Grant, C. A. (1987). An analysis of multicultural education in the United States. Harvard Educational Review, 57(4), 421-444. 
Smetana, J. G. (1988). Adolescents' and parents' conceptions of parental authority. Child Development, 59(2), 321-335. doi:10.2307/1130313

Smetana, J. G., Campione-Barr, N., \& Metzger, A. (2006). Adolescent development in Interpersonal and societal contexts. Annual Review of Psychology, 57(1), 255284. doi:10.1146/annurev.psych.57.102904.190124

Steinberg, L., \& Silverberg, S. B. (1986). The vicissitudes of autonomy in early adolescence. Child Development, 57(4), 841-851. doi:10.2307/1130361

Stoughton E. H., \& Sivertson, C. (2005). Communicating across cultures: Discursive challenges and racial identity formation in narratives of middle school students. Race, Ethnicity \& Education, 8(3), 277-295.

Tadmor, C. T., Hong, Y. Y, Chao, M. M., Wiruchnipawan, F., \& Wang, W. (2012). Multicultural experiences reduce intergroup bias through epistemic unfreezing. Journal of Personality, 103(5), 750-772. doi:10.1037/a0029719

Taylor, E. (2009). The foundations of critical race theory in education: An introduction. In E. Taylor, D. Gillborn, \& G. Ladson-Billings (Eds.), Foundations of critical race theory in education (pp. 1-13). New York. NY: Routledge.

Taylor, S., \& Sidhu, R. K. (2012). Supporting refugee students in schools: What constitutes inclusive education? International Journal of Inclusive Education, 16(1), 39-56.

Tzanakis, M. (2011). Bourdieu's social reproduction thesis and the role of cultural capital in educational attainment: A critical review of key empirical studies. Educate, 11(1), $76-90$. 
United Nations High Commissioner for Refugees. (2005). Refugee status determination: Identifying who is a refugee, self-study module 2. Retrieved from http://www.unhcr.org

United Nations High Commissioner for Refugees. (2015). UNHCR global trends 2014. Retrieved from http://unhcr.org

Uryu, M., Steffensen, S. V., \& Kramsch, C. (2014). The ecology of intercultural interaction: Timescales, temporal ranges and identity dynamics. Language Sciences, 41, 41-59. doi: 10.1016/j.langsci.2013.08.006

Valenta, M. (2010). Refugees' social trajectories and trajectories of the self. Forum: Qualitative Social Research, 11(2), Art. 5.

Verschueren, J. (2008). Intercultural communication and the challenges of migration. Language and Intercultural Communication, 8(1), 21-35. doi: 10.2167/laic298.0

Vertovec, S. (2007). Super-diversity and its implications. Ethnic and racial studies, 30(6), 1024-1054. doi: 10.1080/01419870701599465

Vogt, W. P., Gardner, D. C., \& Haeffele, L. M. (2012). When to use what research design. New York, NY: Guilford.

Volet, S. E., \& Ang, G. (1998). Culturally mixed groups on international campuses: An opportunity for inter-cultural learning. Higher Education Research \& Development, 17(1), 5-23. doi: 10;1080/0729436980170101

Vorhaus, J. (2014). Function and functional explanation in social capital theory: A philosophical appraisal. Studies in Philosophy and Education, 33(2), 185-199. doi: $10.1007 / \mathrm{s} 11217-01309380-5$. 
Wang, M. T., \& Eccles, J. S. (2012). Social support matters: Longitudinal effects of social support on three dimension of school engagement from middle to high school. Child Development, 83(3), 877-895. doi: 10.1111/j.14678624.2012.01745.x

Watkins, P. G., Razee, H., \& Richters, J. (2012). “I'm telling you... the language barrier is the most, the biggest challenge:" Barriers to education among Karen refugee women in Australia. Australian Journal of Education, 56(2), 126-141.

Wentzel, K. R., Baker, S. A., \& Russell, S. L. (2012). Young adolescents’ perceptions of teachers' and peers' goals as predictors of social and academic goal pursuit. Applied Psychology: An International Review, 61(4), 605-633. doi: 10.1111/j.1464-0597.2012.00508.x

Wentzel, K. R., \& Watkins, D. E. (2002). Peer relationships and collaborative learning as contexts for academic enablers. School Psychology Review, 31(3), 366-377.

Yoon, B. (2012). Junsuk and Junhyuck: Adolescent immigrants' educational journey to success and identity negotiation. American Educational Research Journal, 49(5), 971-1002. doi: 10.3102/0002831212443694

Yosso, T. J. (2005). Whose culture has capital? A critical race theory discussion of community cultural wealth. Race Ethnicity and Education, 8(1), 69-91. doi: $10.1080 / 1361332052000341006$

Zimmerman, S. (1995). Perceptions of intercultural communication competence and international student adaptation to an American campus. Communication Education, 44(4), 321-335. 


\section{Appendix A}

\section{Semi-Structured Interview Protocol}

\section{Part 1}

1. Tell me about your day.

2. Tell me about yourself

a. What is your family like? Tell me about your family.

b. Who are your best friends? How did you become friends with them?

3. What do you do when you go home after school? What do you do on the weekends?

4. How long have you lived in the U.S.?

5. Where did you live before coming to the U.S.?

6. What do you remember about your life before coming to the U.S?

Part 2

7. How do you like school?

8. Did you go to school before coming to the U.S.?

9. When you first started school here, what things surprised you?

10. What things do you find confusing about school? Classes? Routines?

11. What are your favorite subjects? What do you like to learn most?

12. What do you dislike about school, both in class and other times (e.g., recess, lunch)?

13. What do you like about school, both in class and other times (e.g., recess, lunch)?

Part 3

14. Do you enjoy working with other students? Why or why not? 
15. Do your best friends go to school with you?

16. If you could choose anyone to work with in your class, who would it be?

17. What makes you choose someone to work with?

18. How do you feel when the teacher has you work with someone you do not want to work with? Do you let the teacher know how you feel?

19. Is there anything else you would like to tell me about that I have not asked? 
Appendix B

\section{Observation Protocol}

1. What are the distinguishing characteristics of the day (e.g., different schedule, infrequent events, weather, new students)?

2. What is the classroom layout?

3. What is the classroom atmosphere?

4. What is the intended content of the lesson?

5. What are the students actually talking about?

6. Describe the students involved in the intercultural interaction (e.g., cultural background, languages spoken, academic achievement, friends or not).

7. Describe the body language of the students involved.

8. Were there any interruptions during the interaction?

9. How long did the interaction last?

10. Was anything accomplished during the interaction?

11. Were there any other interesting characteristics of the interaction? 
Appendix C

Descriptive Codes

Ohma
Simple/ Primitive/ By the land
Identity
Proximity
Detailed Examples
Dichotomy
Friends
Nice/ Kind
Humor
Islam
Struggle
Learning English
Focus/ Learning/ Hard work
Needing help
Misbehaving
Compliance
On task
Fairness
Family

Khalidah

Family

Nice

School

Islam

Dichotomy

Fun

Friends

English

Struggle

Entertainment

Content

Pride

Play

Split identity

On task

Learning

Relationship

Emotion

Compliance

Choice

Shamshidah
Friends
Compliance
Family
Proximity
Moving
Differences
Learning
Future
Content
Islam
Size of school
English
Comfort
Choice
Relationship
Quiet
Nice
Isolation
Nervous
Smart
Ignored
Shy
Help

Shamshidah

Compliance

Family

Proximity

Moving

Learning

Future

Content

Islam

Size of school

English

Comfort

Choice

Relationship

Quiet

Nice

Isolation

Nervous

Smart

Ignored

Help

Common to all three:

Dichotomy

Friends

Nice/ Kind

Islam

Struggle \& Needing Help

Focus/ Learning/ Hard Work

Learning English

Compliance \& On Task

Family 